Florida International University FIU Digital Commons

FIU Electronic Theses and Dissertations

University Graduate School

7-8-2011

\title{
Modeling of Pipeline Transients: Modified Method of Characteristics
}

Stephen L. Wood

Florida International University, swood009@fiu.edu

DOI: $10.25148 /$ etd.FI1 1080807

Follow this and additional works at: https://digitalcommons.fiu.edu/etd

\section{Recommended Citation}

Wood, Stephen L., "Modeling of Pipeline Transients: Modified Method of Characteristics" (2011). FIU Electronic Theses and Dissertations. 456.

https://digitalcommons.fiu.edu/etd/456

This work is brought to you for free and open access by the University Graduate School at FIU Digital Commons. It has been accepted for inclusion in FIU Electronic Theses and Dissertations by an authorized administrator of FIU Digital Commons. For more information, please contact dcc@fiu.edu. 


\title{
FLORIDA INTERNATIONAL UNIVERSITY
}

Miami, Florida

MODELING OF PIPELINE TRANSIENTS:

MODIFIED METHOD OF CHARACTERISTICS

\author{
A thesis submitted in partial fulfillment of the \\ requirements for the degree of \\ MASTER OF SCIENCE \\ in \\ MECHANICAL ENGINEERING \\ by
}

Stephen Wood

2011 
To: Dean Amir Mirmiran

College of Engineering and Computing

This thesis, written by Stephen Wood, and entitled Modeling of Pipeline Transients: Modified Method of Characteristics, having been approved in respect to style and intellectual content, is referred to you for judgment.

We have read this thesis and recommend that it be approved.

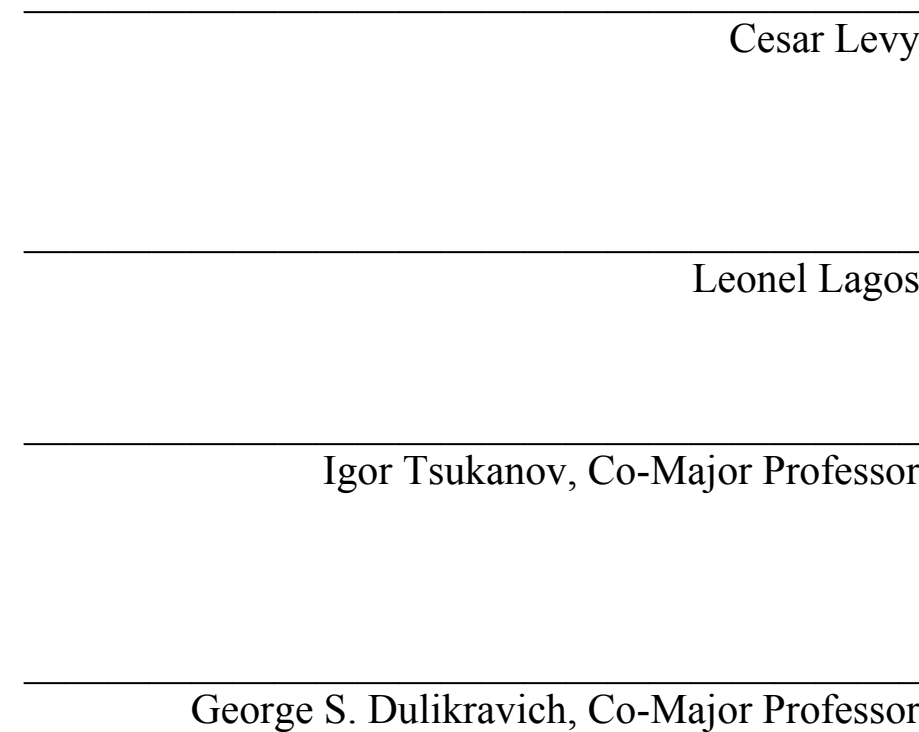

George S. Dulikravich, Co-Major Professor

Date of Defense: July 8, 2011

The thesis of Stephen Wood is approved.

\begin{tabular}{r} 
Dean Amir Mirmiran \\
College of Engineering and Computing \\
\hline Interim Dean Kevin O'Shea \\
University Graduate School
\end{tabular}

Florida International University, 2011 


\section{DEDICATION}

To the Wood, Nunes, and Otis families, especially my mother who tirelessly gave of herself to show her children how to live their dreams. 


\section{ACKNOWLEDGMENTS}

First of all I would like to express my gratitude to Dr. George S. Dulikravich and Dr. Igor Tsukanov, my major professors, for their excellent guidance, encouragement, and for their support in times when I needed it the most. To Dr. Dulikravich and Dr. Lagos, I give my deepest gratitude for welcoming me into their research groups and for the opportunities therein.

I would also like to thank Dr. Cesar Levy, for being part of my academic committee, for the fruitful collaboration during this work, and for being a memorable lecturer. Dr. Seckin Gokaltun and Dr. Dwayne McDaniels provided essential instruction and guidance during the experimental portion of this work, my deepest appreciation goes to them for their good judgment and humor.

I additionally express my thanks to all my teachers, students and colleagues with whom I have had the great pleasure to work with and learn from during these years. I am also grateful to the Department of Mechanical and Materials Engineering for providing me a platform to conduct my research.

I am also thankful to my friends and fellow researchers in the MAIDROC Laboratory and in the DOE Fellow's program. All of whom have contributed to my whole learning experience, in many ways. I'd like to share my particular gratitude to Mr. Denny Carvajal for his assistance with filing paper work.

Especially warm thanks go to my parents Jennifer and Dayton, my Uncle Frederick, my sisters Katherine and Abigail, my wife Kendra and all my family and friends for their constant support, patience and unconditional love during this work. 


\title{
ABSTRACT OF THE THESIS \\ MODELING OF PIPELINE TRANSIENTS: MODIFIED METHOD OF \\ CHARACTERISTICS
}

\author{
by \\ Stephen Wood \\ Florida International University, 2011 \\ Miami, Florida \\ Professor George S. Dulikravich, Co-Major Professor \\ Professor Igor Tsukanov, Co-Major Professor
}

The primary purpose of this research was to improve the accuracy and robustness of pipeline transient modeling. An algorithm was developed to model the transient flow in closed tubes for thin walled pipelines. Emphasis was given to the application of this type of flow to pipelines with small radius $90^{\circ}$ elbows. An additional loss term was developed to account for the presence of $90^{\circ}$ elbows in a pipeline. The algorithm was integrated into an optimization routine to fit results from the improved model to experimental data. A web based interface was developed to facilitate the pre- and postprocessing operations.

Results showed that including a loss term that represents the effects of $90^{\circ}$ elbows in the Method of Characteristics (MOC) [1] improves the accuracy of the predicted transients by an order of magnitude. Secondary objectives of pump optimization, blockage detection and removal were investigated with promising results. 


\section{TABLE OF CONTENTS}

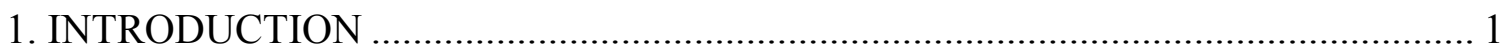

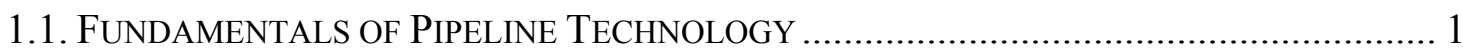

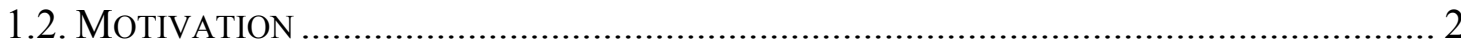

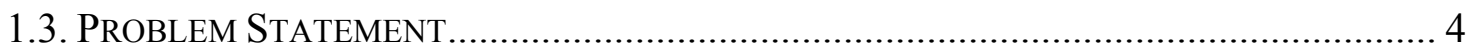

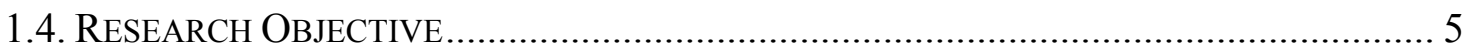

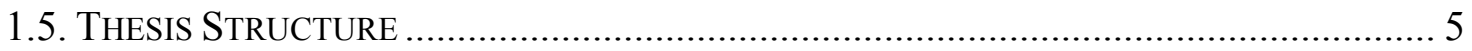

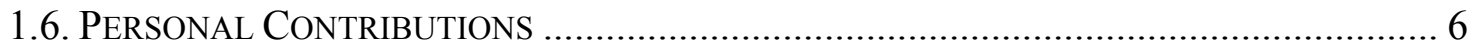

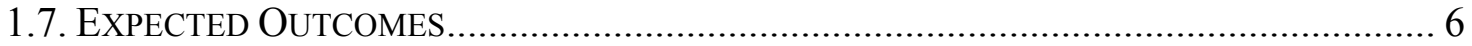

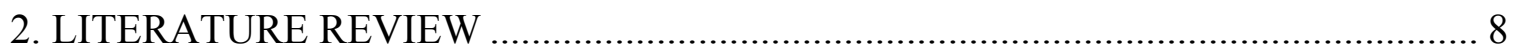

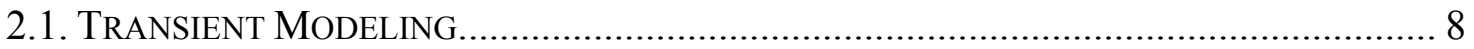

2.1.1. Analytic and Graphic approaches ............................................................ 9

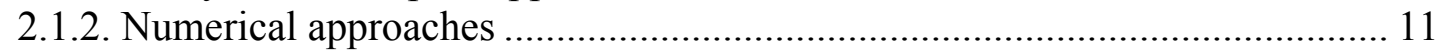

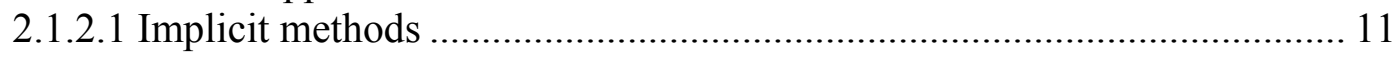

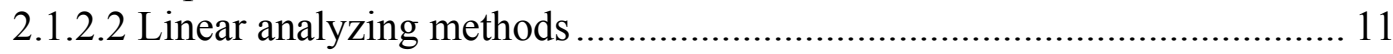

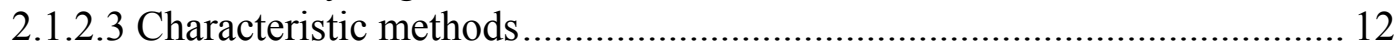

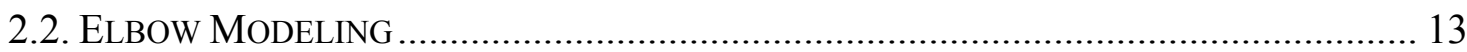

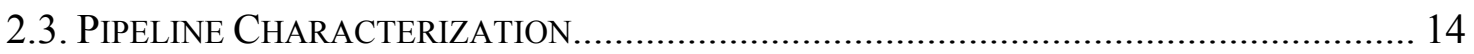

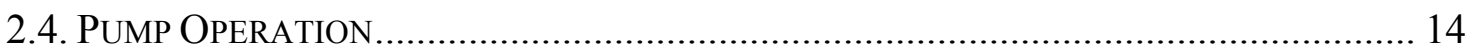

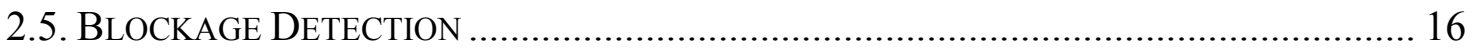

2.6. UNPLUGGING OF COMPLETE BLOCKAGES ………............................................. 17

2.7. WEB APPLICATION FOR ENGINEERING SOFTWARE …......................................... 18

3. PHYSICAL PROBLEM AND MATHEMATICAL FORMULATION …………........ 20

3.1. MATHEMATICAL FoRMULATION FOR TRANSIENT FLOW IN CLOSED CHANNEL ....... 20

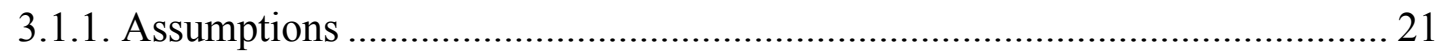

3.1.2. Fluid Bulk Modulus.................................................................................. 22

3.1.2.1 Bulk Modulus for a liquid-air/gas mixture …………………….............. 23

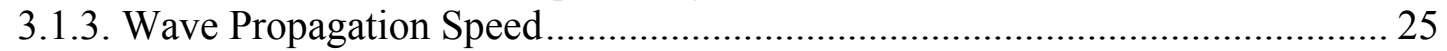

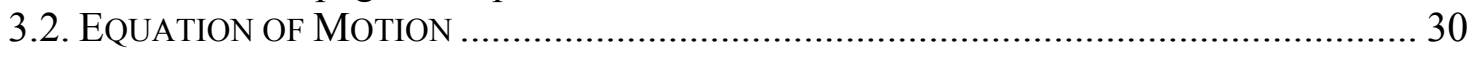

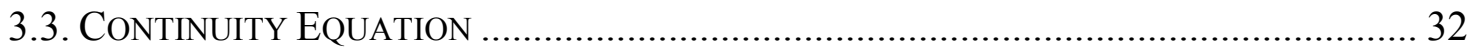

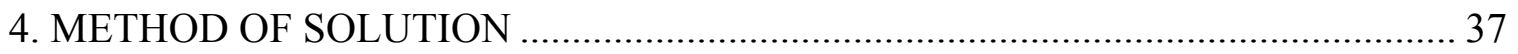

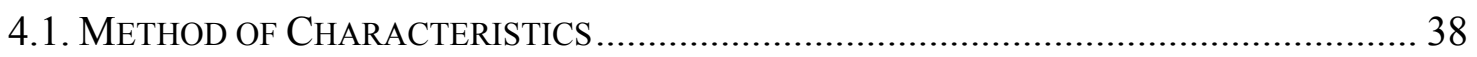

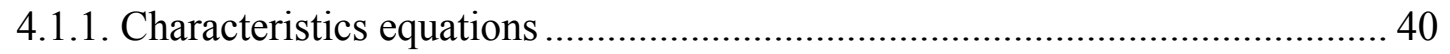

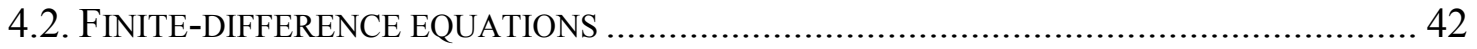

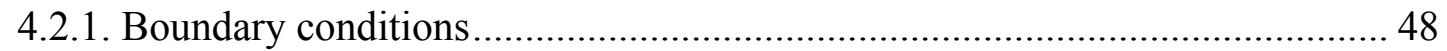

4.2.1.1 Hydraulic unit at the upstream end (pressurization of the system)........... 49

4.2.1.2 Hydraulic unit at the upstream end (depressurization of the system) ........ 50

4.2.1.3 Time Varying Hydraulic unit at the upstream end...................................... 50

4.2.1.4 Dead end at the downstream end .............................................................. 50 
4.2.1.5 Air Pocket at the downstream end ........................................................... 51

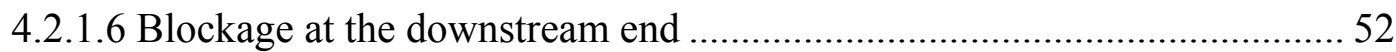

4.3. PIPELINE CHARACTERIZATION THROUGH OPTIMIZATION .........................................53

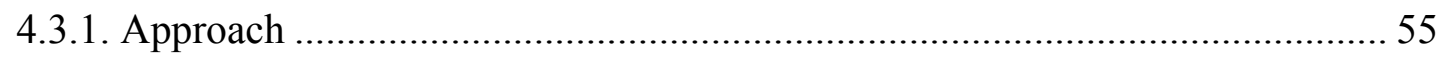

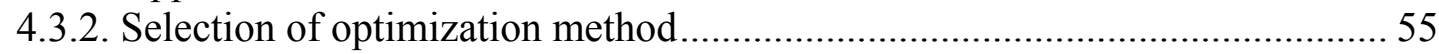

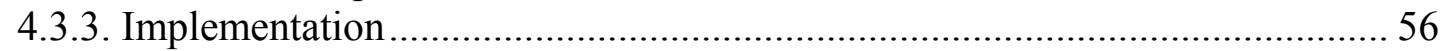

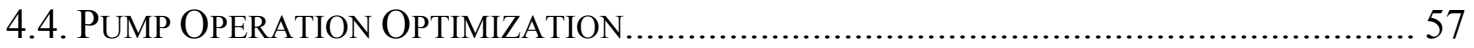

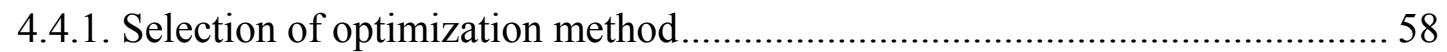

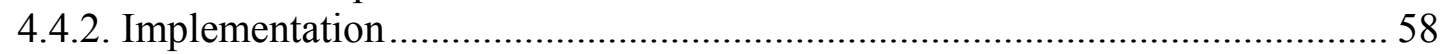

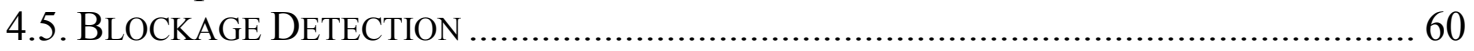

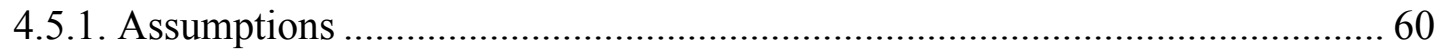

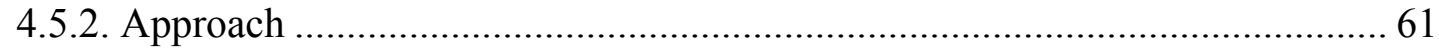

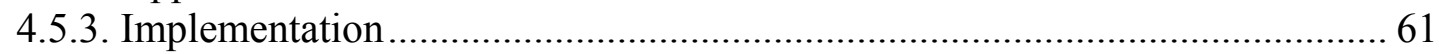

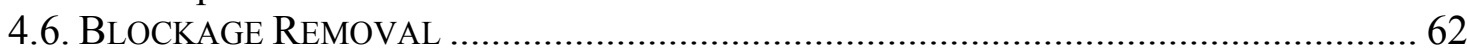

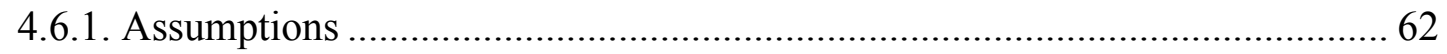

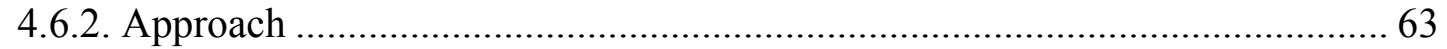

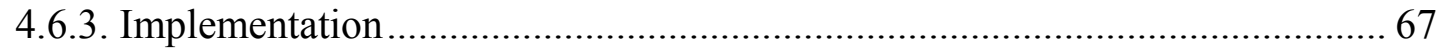

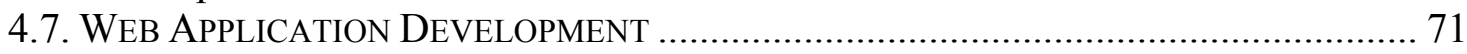

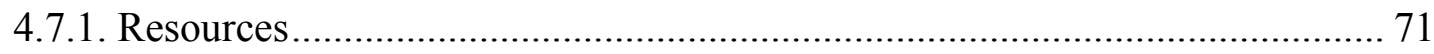

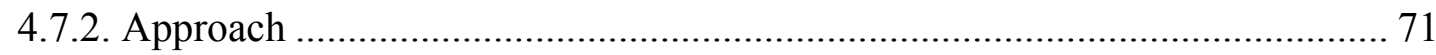

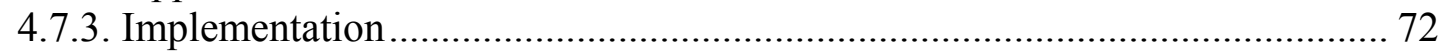

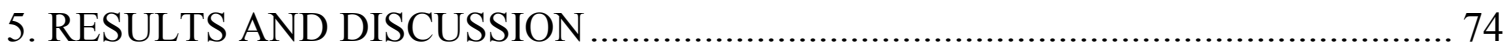

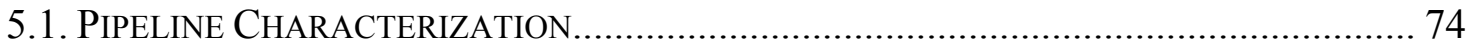

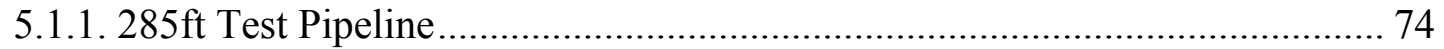

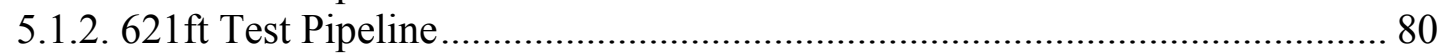

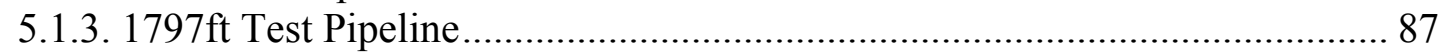

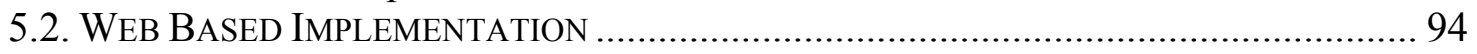

5.3. OPTIMIZATION OF THE INLET HYDRAULIC UNIT OPERATION SCHEDULE................... 96

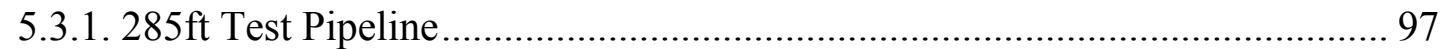

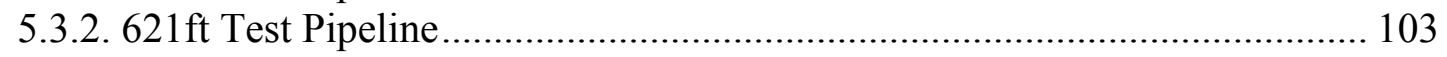

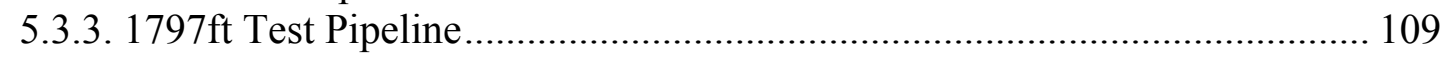

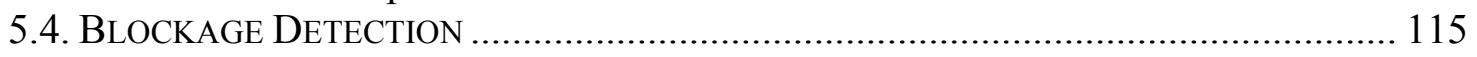

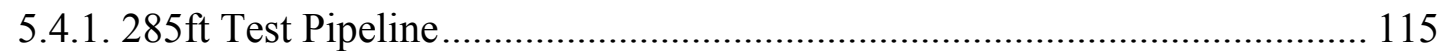

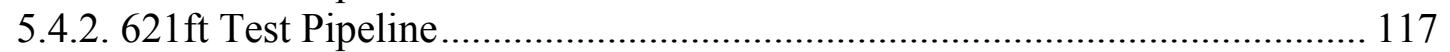

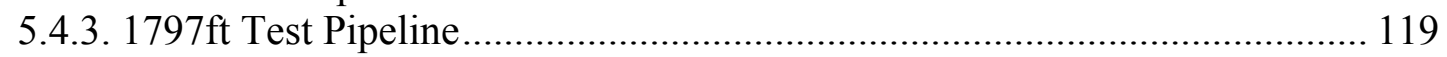

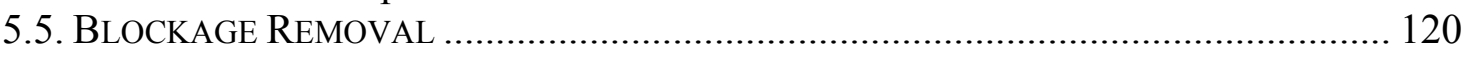

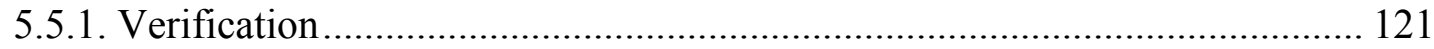

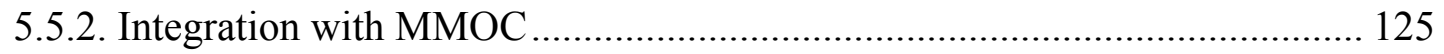

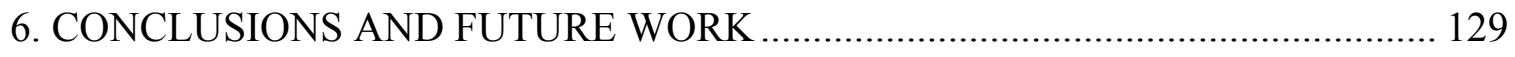


REFERENCES .

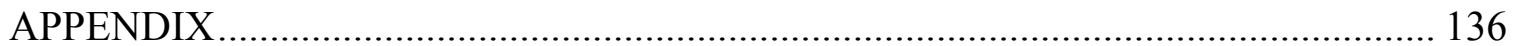




\section{LIST OF FIGURES}

FIGURE

PAGE

Figure 2-1: ApPlicAtion of ARITHMETIC WATER HAMMER EQUATIONS to A Single PIPE.

FIGURE 3-1: (A) INSTANTANEOUS STOPPAGE OF FRICTIONLESS FLUID IN HORIZONTAL PIPE;

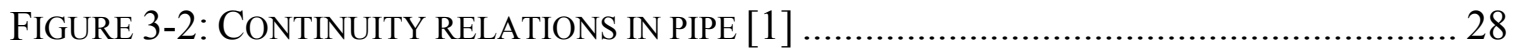

FIGURE 3-3: FREE BODY DIAGRAM FOR APPLICATION OF THE EQUATION OF MOTION [1] ... 30

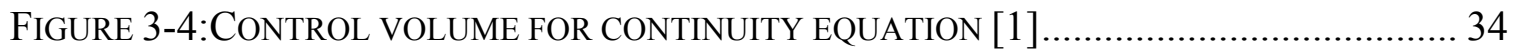

Figure 4-1: CHARACTERISTICS CURVES IN THE CARTESIAN COORDINATES SYSTEM [1] .... 39

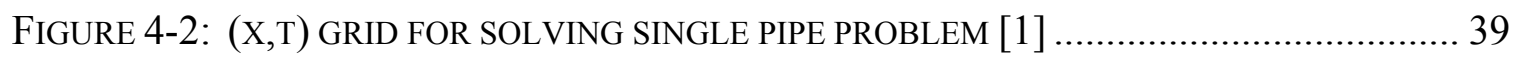

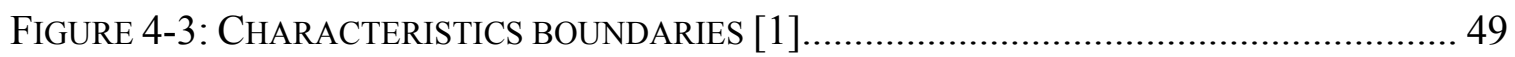

FIGURE 4-4: PIPELINE CHARACTERIZATION FLOW CHART ............................................ 56

FIGURE 4-5: PUMP OPERATION OPTIMIZATION FLOW CHART ......................................... 59

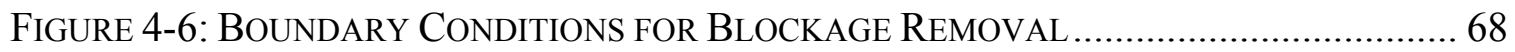

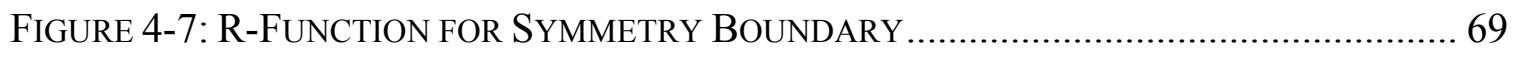

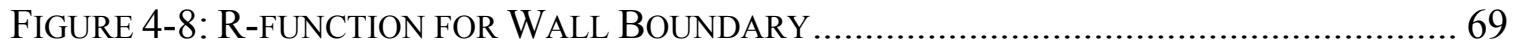

Figure 4-9: COMBINED R-FunCTION FOR BLOCKAGE IN THE RADIAL DiRECTION ........... 70

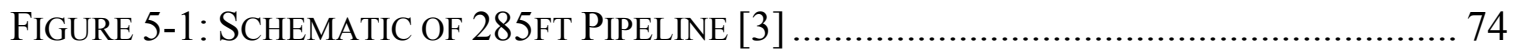

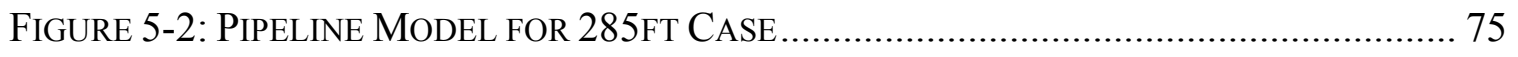

FIGURE 5-3: COMPARISION OF MOC AND EXPERIMENTAL RESULTS FOR NUVISION 285FT

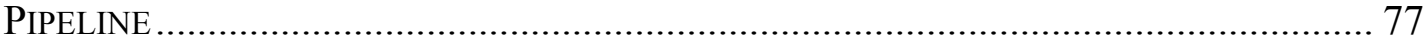

FIGURE 5-4:COMPARISION OF MMOC AND EXPERIMENTAL RESULTS FOR NUVISION

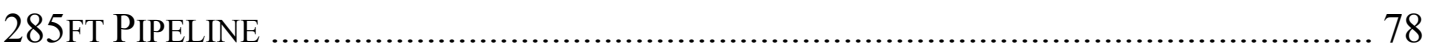

Figure 5-5: Characterization of NuVision 285Ft PiPELINE By Pressure ............... 79 
Figure 5-6: Characterization OF NuVision 285FT Pipeline by TiMe ERroR 80

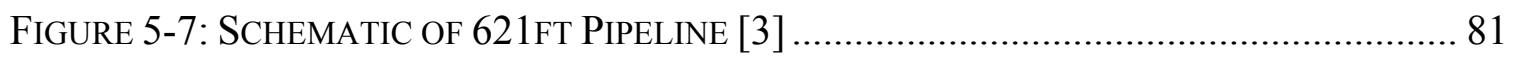

Figure 5-8: PIPELINE Model fOR 621FT CASE........................................................ 82

FIGURE 5-9:COMPARISION OF MOC AND EXPERIMENTAL RESULTS FOR NUVISION 621FT PIPELINE. 84

FIGURE 5-10:COMPARISION OF MMOC AND EXPERIMENTAL RESULTS FOR NUVISION 621FT PIPELINE 86

Figure 5-11: Characterization Of NuVision 621ft PiPELINE BY Pressure .............. 86

Figure 5-12: Characterization of NuVision 621ft PiPELINE By Time ERror .......... 87

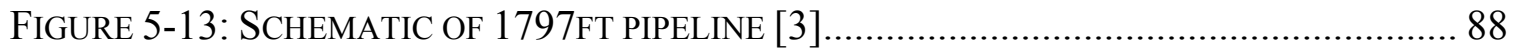

FigURE 5-14: PIPELINE MOdEL FOR 1797FT CASE.......................................................... 89

FIGURE 5-15:COMPARISION OF MOC AND EXPERIMENTAL RESULTS FOR NUVISION

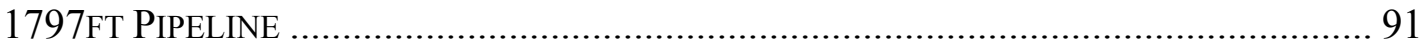

FIGURE 5-16:COMPARISION OF MMOC AND EXPERIMENTAL RESULTS FOR NUVISION 1797FT PIPELINE 92

Figure 5-17: CHARACTERIZATION OF NUVision 1797Ft PiPELINE By PRESSURE ERror 93

Figure 5-18: Characterization Of NuVision 1797Ft PiPeline By Time ERror ........ 93

FIGURE 5-19: WEB FORM FOR PIPELINE CONFIGURATION .............................................. 94

FigURE 5-20: WEB FoRM FOR BOUNDARY CONDITION SELECTION ................................. 95

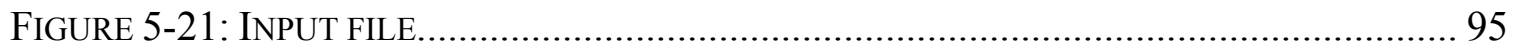

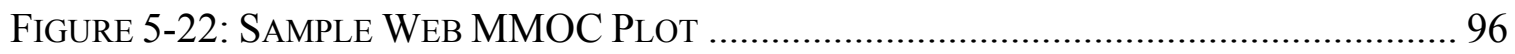

FIGURE 5-23: PUMP OPERATION OPTIMIZATION FOR NUVISION 285FT PIPELINE BY

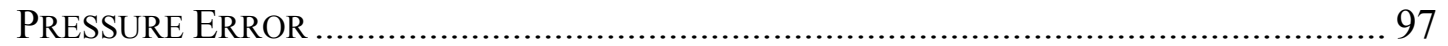

Figure 5-24: Pump Operation OptimizATION FOR NuVision 285FT PiPELINE By Time

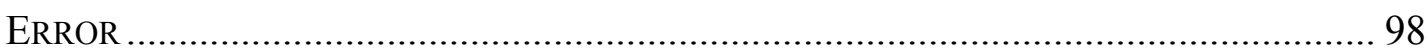


Figure 5-25: Initial Pump Optimization Results for 285Ft CASE............................. 100

FiguRE 5-26: INTERIM PuMP OPTIMIZATION RESUltS For 285FT CASE .......................... 101

Figure 5-27: Final Pump OPTIMIZATION RESULTS FoR 285Ft CASE .............................. 102

Figure 5-28: Pump OPERATION Optimization FOR NuVision 621 fT PIPELINE BY

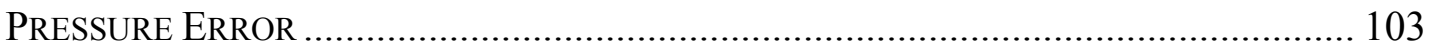

Figure 5-29: Pump OPERATION OptimizATION FOR NuVision 621 fT PIPELINE BY TIME ERROR 104

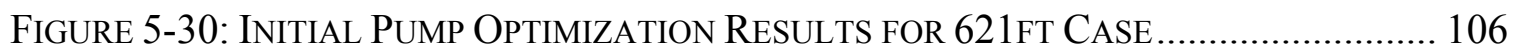

Figure 5-31: INTERIM PumP OptIMIZATION RESUltS For 621Ft CASE .......................... 107

Figure 5-32: Final Pump Optimization Results for 621ft CASE .............................. 108

Figure 5-33: Pump Operation Optimization for NuVision 1797ft Pipeline by

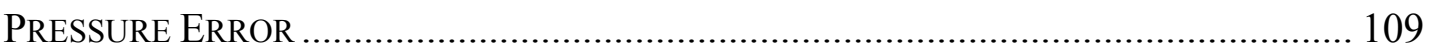

Figure 5-34: Pump Operation OPtIMIZATION FOR NuVision 1797FT Pipeline BY

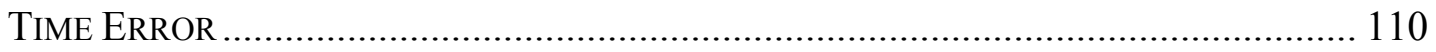

FiguRe 5-35: INITIAL PUMP OPTIMIZATION RESULTS FoR 1797Ft CASE........................... 112

FigURE 5-36: INTERIM PUMP OPTIMIZATION RESULTS FOR 1797fT CASE ......................... 113

Figure 5-37: Final Pump Optimization Results for 1797Ft CASE ............................. 114

Figure 5-38: Initial PiPeline for Blockage Detection in 285Ft CASE ..................... 115

Figure 5-39: Blockage DeteCtion Results For 285Ft CASE..................................... 116

Figure 5-40: Initial Pipeline for Blockage Detection in 621 ft CASe ..................... 117

FiguRE 5-41: BlocKage DeteCtion ResultS For 621ft CASE..................................... 118

Figure 5-42: Initial Pipeline for Blockage Detection in 1797ft CASe .................. 119

Figure 5-43: Blockage Detection Results For 1797ft CASE................................... 120

FiguRE 5-44: VERIFICATION OF RADIAL DiSPLACEMENT DiSTRIBUTION........................... 122 
FigURE 5-45: VerificATION OF RADIAL DisPLACEMENT DistribUTION SHOWN IN

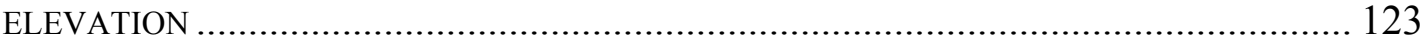

Figure 5-46: VerificATION OF AXIAL DisPlaCEMENT Distribution.............................. 123

Figure 5-47: Verification of AXIAl Displacement Distribution SHOWN IN

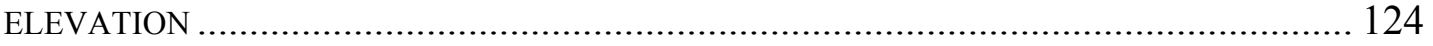

Figure 5-48: MMOC Pipeline Model for Blockage Removal for 285Ft CASE.... 126

Figure 5-49: Pressure History for Blockage Removal in 285Ft CASE.................. 127

FigURE 5-50: BLOCKAGE MOTION FOR 285FT CASE_.................................................. 128 


\section{LIST OF TABLES}

TABLE

PAGE

TABLE 2: PIPELINE CHARACTERIZATION DESIGN VARIABLES ....................................... 57

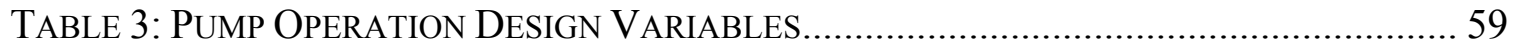

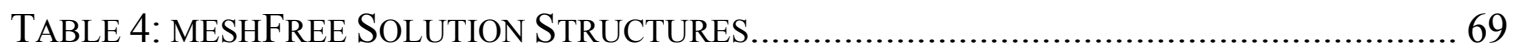

TABLE 5: COMPARISON OF MOC AND MMOC FOR 285 FT CASE ................................... 76

TABLE 6: COMPARISON OF MOC AND MMOC FOR 621 FT CASE ................................... 83

TABLE 7: COMPARISON OF MOC AND MMOC FOR 1797 FT CASE .................................. 89

TABle 8: Pump Operation Optimization Summary FOR 285FT CASE ........................ 99

TABle 9: Pump Operation Optimization Summary For 621Ft CASE ....................... 105

TABLE 10: Pump OPERATION Optimization SuMMARY FOR 1797FT CASE .................... 111

TABLE 11: CONVERGENCE OF DiSPLACEMENT ........................................................ 121

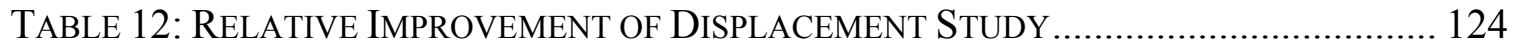

TABle 13: PARAMETERS For 285Ft Blockage Removal CASE ................................. 125 
Nomenclature

\begin{tabular}{|l|l|}
\hline $\mathrm{a}(\mathrm{m} / \mathrm{s})$ & Wave speed \\
\hline $\mathrm{A}$ & Cross-sectional area of pipe line \\
\hline $\mathrm{c}_{1}$ & Support coefficient of pipe line \\
\hline $\mathrm{c}_{\mathrm{g}}$ & Coefficient of geometric impedance \\
\hline $\mathrm{D}(\mathrm{m} / \mathrm{s})$ & Pipe diameter \\
\hline $\mathrm{E}(\mathrm{Pa})$ & Modulus of elasticity of the pipe wall \\
\hline $\mathrm{e}(\mathrm{m})$ & Pipe wall thickness \\
\hline $\mathrm{f}$ & Darcy-Weisbach friction factor \\
\hline $\mathrm{g}\left(\mathrm{m} / \mathrm{s}^{2}\right)$ & Gravitational acceleration \\
\hline $\mathrm{H}(\mathrm{m})$ & Instantaneous piezometric head \\
\hline $\mathrm{H}_{\mathrm{A}}(\mathrm{m})$ & Absolute head \\
\hline $\bar{H}(\mathrm{~m})$ & Barometric head \\
\hline $\mathrm{H}_{\mathrm{t}}(\mathrm{m} / \mathrm{s})$ & $\partial H / \partial t$ \\
\hline $\mathrm{H}_{\mathrm{x}}$ & $\partial H / \partial x$ \\
\hline $\mathrm{K}(\mathrm{Pa})$ & Bulk modulus of elasticity of the fluid and entrained air \\
\hline $\mathrm{l}$ & Multiplier in method of characteristics \\
\hline $\mathrm{m}$ & Poisson's ratio of the pipe wall \\
\hline $\mathrm{r}\left(\mathrm{kg} / \mathrm{m}^{3}\right)$ & Mass density of fluid \\
\hline $\mathrm{s}_{\mathrm{j}}(\mathrm{m})$ & Length of the jth straight section of the pipeline \\
\hline $\mathrm{V}(\mathrm{m} / \mathrm{s})$ & Instantaneous velocity \\
\hline $\mathrm{V}_{\mathrm{t}}\left(\mathrm{m} / \mathrm{s}^{2}\right)$ & $\partial V / \partial t$ \\
\hline $\mathrm{V}_{\mathrm{x}}(1 / \mathrm{s})$ & $\partial V / \partial x$ \\
\hline $\mathrm{V}\left(\mathrm{m}^{3}\right)$ & Volume of trapped air \\
\hline
\end{tabular}




\section{CHAPTER I}

\section{INTRODUCTION}

Pressure and flow rate oscillations, transients, occur in pipelines and control systems operated in processes vital to societies' resource utilization worldwide. Accurate modeling of the transient phenomenon which occur in such systems enables efficient and safe operation which reduces costs, the occurrence of accidents and the likely-hood of adverse environmental impacts.

\subsection{Fundamentals of Pipeline Technology}

The term pipe is defined herein as a closed conduit of circular cross section made of steel. The term pipeline refers to a long line of connected segments of pipe, with pumps, valves, control devices, and other equipment/facilities necessary for operating the system. It is intended for transporting a fluid (liquid or gas), mixture of fluids, solids or fluid-solid mixture [2]. Unless otherwise specified, the pipelines discussed in this thesis have a diameter of 3 inches $(7.62 \mathrm{~cm})$.

Water hammer (or, more generally, fluid hammer) is a pressure surge or wave resulting when a fluid (usually a liquid but sometimes also a gas) in motion is forced to stop or change direction suddenly (momentum change). Water hammer commonly occurs when a valve is closed suddenly at an end of a pipeline system, and a pressure wave propagates in the pipe.

This pressure wave can cause major problems for the system, from noise and vibration to pipe collapse. It is possible to reduce the effects of the water hammer pulses with accumulators and other features. 
Pipelines experience severe dynamic forces during a water hammer event. When these forces make the system move significant fluid structure interaction (FSI) may occur. When FSI takes place the liquid and pipe systems cannot be treated separately in a theoretical analysis: interaction mechanisms have to be taken into account.

\subsection{Motivation}

In this work the primary application considered is the transfer of High Level Waste (HLW) at Department of Energy (DOE) sites in the United States of America. In the past, some of the pipelines at DOE sites have plugged during HLW transfers, resulting in schedule delays and increased costs. Availability of a pipeline unplugging tool/technology is crucial to ensure smooth operation of the waste transfers and to ensure tank farm cleanup milestones are met. Florida International University's (FIU) Applied Research Center (ARC) has previously tested and evaluated various unplugging technologies through an industry call. Based on mockup testing, two technologies were identified that could withstand the rigors of operation in a radioactive environment and had the ability to handle small radius $90^{\circ}$ elbows. These technologies were NuVision Engineering's Fluidic Wave-action Technology and AIMM Technologies' Hydrokinetics. As a DOE Fellow in the DOE/FIU Science \& Technology Workforce Development Initiative, I participated in the technology evaluation by preparing stimulant blockages, constructing the test apparatus and assisting to conduct the tests.

The testing and qualification was comprised of a heavily instrumented 3-inch diameter full-scale pipeline, facilitating extensive data acquisition for design optimization and performance evaluation as it applies to three types of plugs typical of DOE HLW. 
One of these plug types is a kaolin-water mixture typically used in emulating slurry mixes. The other two plug types are crystallized salt plug simulants recommended by Hanford Waste Treatment Plant (WTP) engineers. Three different test bed lengths (285, 621, and $1797 \mathrm{ft}$.) were utilized to determine the effectiveness of NuVision's technology with respect to pipe length. Erosion rates were determined for each plug type and at each test bed length. An amplification of the inlet pressure was observed at the blockage area that demonstrated the need for a complete analysis of the pressure pulse propagation through the pipeline. Wave speeds have also been analyzed to determine correlations between the amplification factors, unplugging rates and equipment control parameters utilized by NuVision [3].

Cross-site lines at Hanford can extend almost eight miles. With access locations at either end, maximum pipe lengths to plugs could reach as far as 19,000 ft. from an entry point. For this reason, the experimental test data was extrapolated to $19,000 \mathrm{ft}$. Extrapolated test data include maximum pressure, unplugging rates, energy input, and wave speeds. During the experimental testing, variations in the process control parameters were observed and became more extreme at the longer test bed length. These variations are believed to be due to changes in the environmental conditions during testing. The variability in process control parameters makes it difficult to extrapolate test results to longer pipeline lengths [3].

An alternative method to predict some parameters of the testing at the scaled up pipe lengths is to use a simplified quasi-linear model of the transient flow in the pipe based upon the Method of Characteristics (MOC). 


\subsection{Problem Statement}

Safe and efficient operation of pipeline systems is a challenging dynamic task. It is difficult to anticipate or predict the effects of changing pump, valve, tank, or other facility states. It is also difficult to determine how quickly the operational state of a pump, valve, tank, or other facility can be altered without causing damage or unacceptable performance elsewhere in a pipeline [2]. Carrying out experiments to evaluate the various operational scenarios is time consuming, costly and the results may not apply to unanticipated situations.

There is a lack of software capable of characterizing a pipeline by determining the damping parameters in the model in a timely fashion [4]. The damping parameters in a model represent the energy lost as a pressure wave propagates in a pipeline due to the fluid properties, the internal reflections caused by the geometry of the pipeline, and the vibration of the pipeline.

There are at least three further functions of a software package that are highly desired by pipeline operators which have not yet been implemented. These capabilities are:

- $\quad$ predicting the necessary pump or reservoir operation schedule to achieve desired pressures or flow rates elsewhere in the pipeline in a timely fashion

- locating blockages within pipelines

- modeling and predicting blockage extrusion by fluid forces 


\subsection{Research Objective}

The primary purpose of this research is to improve the accuracy and robustness of pipeline transient modeling. An algorithm is developed to model the transient flow in closed tubes for thin walled pipelines from the Method of Characteristics (MOC) [1]. Emphasis is given to the application of this type of flow to pipelines with small radius $90^{\circ}$ elbows.

Secondary objectives are to:

2.1) Validate the integration the developed algorithm into an optimization routine to enable the timely determination of damping parameters which characterize the pipeline.

2.2) Validate the integration of the developed algorithm with an optimization routine to enable the timely determination of the necessary pump or reservoir operation schedule to achieve desired pressures or flow rates.

2.3) Validate the integration of the developed algorithm with an optimization routine to enable the timely location of full blockages in a pipeline.

2.4) Verify the integration of the developed algorithm with a developed Finite Element Method model of a pipeline blockage for the purpose of modeling and predicting blockage extrusion by fluid forces.

\subsection{Thesis Structure}

In Chapter 2 a literature review of the past and current work in transient modeling, elbow modeling, pipeline characterization, pump operation, blockage detection, pipeline unplugging, and the deployment on engineering software through web 
applications is presented. In Chapter 3 the physical problems and their mathematical formulations are presented. In Chapter 4 the method of solution for the physical problems is presented. In Chapter 5 the results and discussions for each of the physical problems is presented. In Chapter 6 the conclusions reached in this work are presented. The references utilized in the work are listed in Chapter 7. A code snippet is provided for the MMOC air pocket at the downstream end boundary condition in the appendix.

\subsection{Personal Contributions}

1. A loss term to account for the effect of $90^{\circ}$ elbows on pipeline transients: $G_{j}=\frac{c_{g}}{s_{j}}$

2. Coupling of the MMOC solver with a differential evolutionary optimization algorithm for user specified parameters and objectives

3. Coupling of the MMOC solver with a blockage locating routine

4. Coupling of the MMOC solver with a solid mechanics solver, meshFree [5], to enable modeling of blockage removal

5. Web-based tool which facilitates users setting up and viewing results of pipeline transients simulations

\subsection{Expected Outcomes}

1. The inclusion of a loss term which accounts for the effect of $90^{\circ}$ elbows on pipeline transients will improve accuracy of peak pressures and wave form shapes predicted by the MMOC code

2. Optimization through the use of a differential evolutionary algorithm will enable characterization of a pipeline through the determination of the friction factor, $\mathrm{f}$, 
and the coefficient of geometric impedance, $\mathrm{c}_{\mathrm{g}}$

3. Optimization through the use of a differential evolutionary algorithm will enable determination of time varying inlet reservoir pressures for user specified objectives such as blockage extrusion

4. The web-based tool will enable the timely modeling and analysis of pipeline transients 


\section{CHAPTER II}

\section{LITERATURE REVIEW}

\subsection{Transient Modeling}

The first significant contributions to the study of wave propagation in 'incompressible' fluids were made by Newton and later by Laplace $[4,6]$ who related the speed of sound in air to the pressure and density under the assumptions of isothermal and isentropic compression respectively. These relations, or equations of state, were complemented by the development of the equations of motion for a compressible fluid. Their development was due to the work on inviscid flow by Euler in 1755, and to the later addition of frictional resistance terms by Navier in 1827 and by Stokes in 1845 . It was Stokes who introduced the coefficient of viscosity and presented the momentum equations in their currently accepted form. Early attempts to analyze observed surge and water hammer effects were not directly based upon these fundamental equations, but were based upon the one-dimensional wave equation, originally derived and solved by d'Alembert around 1750 [6].

Various methods of analysis were developed for the problem of transient flow in pipes from the pioneering efforts of Newton, Laplace, Euler, Navier, Stokes and d'Alembert. They range from approximate analytical approaches whereby the nonlinear friction term in the momentum equation is either neglected or linearized, to numerical solutions of the nonlinear system. The Method of Characteristics which converts the two partial differential equations (PDE's) of continuity and momentum into four different 
ordinary differential equations and solves them numerically using finite difference techniques is the most popular approach for handling hydraulic transients [7].

In the majority of the analyses reviewed, the pipes are slender, thin-walled, straight, prismatic and of circular cross-section. The liquid and the pipe-wall material are assumed linearly elastic and cavitation is assumed not to occur. The theories developed are valid for long (compared to the pipe diameter) wavelength, acoustical (convective velocities neglected) phenomena. Important dimensionless parameters in FSI analyses are (i) the Poisson ratio, (ii) the ratio of pipe radius to pipe-wall thickness, (iii) the ratio of fluid mass density to pipe-wall mass density, and (iv) the ratio of fluid bulk modulus to pipe-wall Young's modulus $[2,8]$.

\subsubsection{Analytic and Graphic approaches}

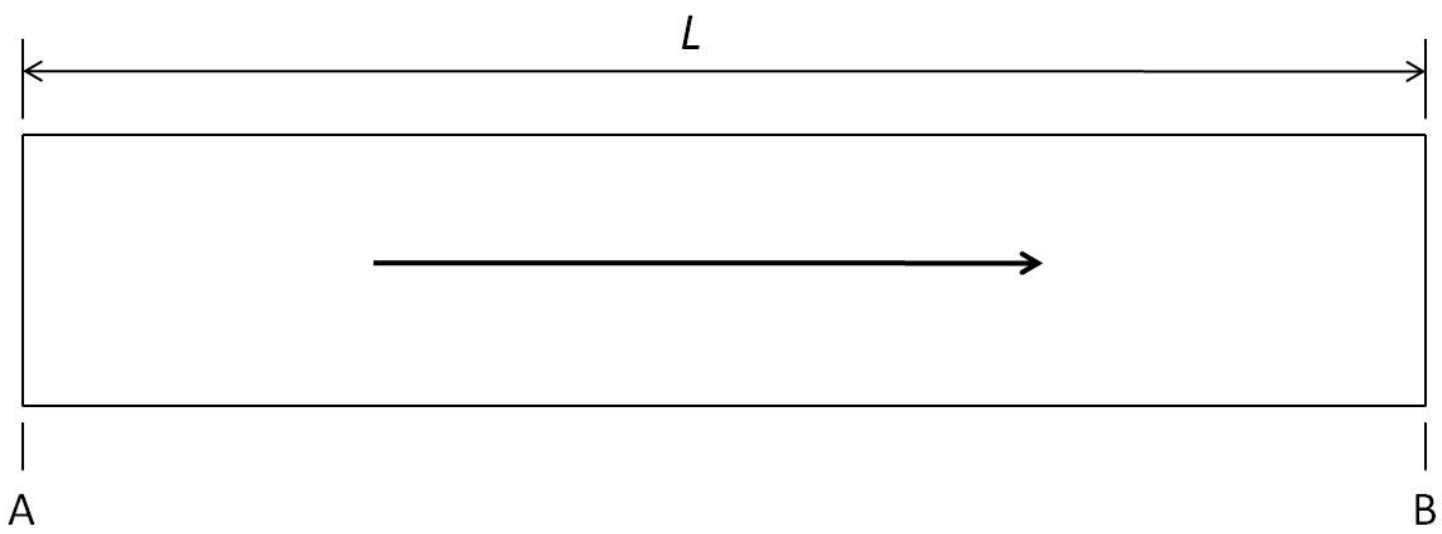

Figure 2-1: Application of Arithmetic Water Hammer equations to a single pipe

Analytic methods often neglect friction and minor losses to simplify the unsteady momentum and continuity equations [6,9]. The reduced momentum equation is stated as

$$
\sum \Delta H= \pm \frac{a}{g} \Delta V
$$


The plus sign is taken for a pressure wave traveling upstream from B to A (Figure 2-1) as from a sudden valve closure and takes the form

$$
H_{A}+\frac{a}{g} V_{A}=H_{B}+\frac{a}{g} V_{B}
$$

Conditions at A occur $L / a$ seconds after the conditions at B. With $H_{B}, V_{B}$ known, then one additional piece of information known at A, $L / a$ seconds from a boundary condition permits $H_{A}$ and $V_{A}$ to be determined. For a wave traveling downstream from $\mathrm{A}$ to $\mathrm{B}$

$$
H_{A}-\frac{a}{g} V_{A}=H_{B}-\frac{a}{g} V_{B}
$$

In which conditions at $\mathrm{A}, H_{A}, V_{A}$ occur $L / a$ seconds before $H_{B}, V_{B}$. From the application of this pair of equations many times, plus the required boundary conditions (such as a reservoir, a valve, or a dead end), the transient solution is developed and solved. This method was used until the early 1930's, when the graphical methods were developed [1].

Graphical water hammer solutions neglect friction in their theoretical development, but utilize means to take it into account by a correction. The integrated $\Delta H= \pm a g \Delta V$ (2.1) may be adapted to a graphical solution, since they plot as straight lines on an HV-diagram. Graphical methods were used as the principal way of solving transient problems from the early 1930 's to the early 1960 's. They have been generally supplanted by digital computer methods $[1,4,6]$. 


\subsubsection{Numerical approaches}

\subsubsection{Implicit methods}

The centered implicit method is a finite difference procedure that can be used successfully for the solution of a class of unsteady fluid flow problems. Its broadest application is in unsteady free surface flow calculations [10]. This procedure is particularly applicable in situations where inertial forces are not as important as the storage or capacitance effects. The method is formulated in such a way that the requirement to maintain a specific relationship between the time increment $\Delta t$ and the length increment $\Delta x$ is relaxed. This feature offers the opportunity for a more flexible scheme than other methods in dealing with complex systems, however, it is necessary to simultaneously solve for all of the unknowns in the system at each time step. When applied to water hammer problems it is necessary to adhere to the Courant-Friedrichs Lewy condition in the time step-distance interval relationship in order to maintain a satisfactory level of accuracy. In these cases the advantage of the method are lost and other methods are recommended [1].

\subsubsection{Linear analyzing methods}

By linearizing the friction term, and dropping other nonlinear terms in the equation of motion, an analytical solution to the equations may be found for sine wave oscillations. These analyses may be considered in two categories: steady-oscillatory fluctuations set up by some forcing function such as by a positive displacement pump; and free vibrations of a piping system. This latter method does not inquire into the nature of the forcing functions, but determines the natural frequencies of the system, and provides information on the rate of damping of the oscillations when forcing is 
discontinued. By the means of harmonic analysis, complex periodic forcing functions may be decomposed into a family of sine wave motions. Each of these may be handled by the momentum and continuity equations, then the solutions added to yield the complete solution $[1,6]$.

\subsubsection{Characteristic methods}

The method of characteristics converts the two partial differential equations of motion and continuity into four total differential equations. These equations are then expressed in finite difference form, using the method of specified time intervals, and solutions are carried out with the use of a computer [4].

The characteristic method has many advantages:

1. Stability criteria are firmly established.

2. Boundary conditions are easily programmed.

3. Minor terms may be retained if desired.

4. Very complex systems may be handled.

5. It has the best accuracy of the finite difference methods.

6. Programs are easy to debug because steady state satisfies all conditions, and an error in programming shows up as a change from steady state.

7. It is a detailed method which allows the print out of complete tabular results [1]. 


\subsection{Elbow Modeling}

In Wood \& Chao a valuable series of tests was carried out on $30^{\circ}, 60^{\circ}, 90^{\circ}, 120^{\circ}$ and $150^{\circ}$ miter bends and on a $90^{\circ}-90^{\circ} \mathrm{T}$-junction. No attempt was made to model the structure; measured junction velocities were used as input to the analysis. It was shown that a single rigidly supported elbow had a negligible influence on pressure waves, whereas unrestrained elbows affected them considerably $[11,12]$. Jones \& Wood gave an analytically derived expression for the junction-coupling induced pressure oscillations around Joukowsky's value in the case of rapid valve closure downstream in a single pipe [13]. The pipe was regarded as a spring-mass system. Calculated results were compared with measurements in an unrestrained vertical pipe. In 1994 and 1996 Tijsseling presented experimental and numerical results on a one-elbow pipe system where the concentrated cavity model was incorporated in the FSI eight equation model. Little has been published on the effect of multiple elbows have on pipeline transients due to the multitude of possible pipeline configurations [14]. The attention has rather been on the importance of rigid supports for pipelines and junctions and the effect of flexible supports on pipeline transients. Dzodzo et al. published full 3D CFD simulation results in 2006 that clearly indicate the large effects small radius elbows have on pipeline flows. Notably the results show that the spacing and relative orientation of the elbows in the pipeline both effect the velocity and pressure profiles for many pipe diameters downstream [15]. 


\subsection{Pipeline Characterization}

The comparison of analytic and numerical simulations with experimental results for the purpose of validating a method has been done extensively by Wylie, Streeter, Wood, Chao, Tijsseling and nearly every other contributor to the field $[1,4,13,14]$. In these studies the experimental apparatus was established and preliminary work was done to establish the parameters that represented the losses in the system, that is the friction factor, $f$, the support coefficient(s), $c_{1}$, the volume of trapped air, $\forall$, or the maximum allowed air release (not considered in this work). In these publications the researchers frequently note the influence of these parameters on simulations and present results from a selection of the parameter values which best fit or bracket the experimental results [1, 11, 16 - 19]. Little has been published on the development of a procedure for determining the loss parameters for a pipeline system. The dominant approach has been to conduct preliminary experiments at steady state conditions to determine the range of parameters representing losses in isolated components of a particular experimental setup. Values within these ranges have then been utilized in the model to achieve the greatest agreement with experimental results. In 2005, Ghidaoui highlights the success of inverse design methods applied to structural engineering problems of system identification and damage detection and to identify parameters in $2 \mathrm{D}$ ground water flow and suggest that similar approaches could be employed for complex piping systems [20].

\subsection{Pump Operation}

The kinematics and dynamics of flow through pumps, especially reciprocating pumps, employed in pipeline systems have been extensively studied by Streeter, Wylie, 
Tullis and others $[1,22,23]$. The focus of these examinations has been on modeling the short duration transients produced by the cyclic pressure variation of the pump's discharge pressure. The often high frequency pulsation generated by pump operation can produce pressure waves with wave lengths near the length of the pipeline diameter. When transient wavelengths and the pipe diameter are of the same order of magnitude it is necessary to consider frequency dependent friction factors in the model of the pipeline $[1,4,21,23-25]$.

In long and complex pipelines such as transport and distribution systems the use of holistic procedures such as the Transient Risk Assessment Procedure (TRAP) to design pipeline systems and guide the operation pumps was prevalent until the early 1990's [23]. In the early 1990's genetic algorithms (GA) rose in popularity in nearly every engineering discipline including the study of hydraulics and pipeline transients [26] as a method of determining design variables to achieve desired objectives. The applications of GA's to pipeline transients can be grouped into three categories, system design, leak detection and calibration, and optimization to minimize expenditure on power $[20,24,25]$. GA's have been successfully utilized to improve the accuracy of pipeline models, to aid engineers in isolating leaks and to provide long term operational schedules to provide services when needed by operating pumps when electricity is most affordable [27].

There has been little work published on the application of GA's or other optimization techniques to short term pump operation in order to rapidly achieve desired effects within a pipeline. Holistic methods and best practices have continued to be the 
prevalent methods used in this area because most pipeline systems are under instrumented [27]. In most long pipelines a rapid response requires a high amplitude transient which can be harmful to the pipeline and facilities connected to it. A rapid response to the desired effect can be ensured with accurate instrumentation and a calibrated model.

\subsection{Blockage Detection}

Blockage development is a common problem in pipeline and pipe network systems for the energy, chemical, and water industries. A blockage can be formed either by localized chemical or physical deposition or by a (negligently) partially closed valve. The existence of a pipeline blockage not only reduces the operation efficiency of a pipeline system, but it can cause severe safety problems if the blockage is not identified in a timely manner [28]. The efforts of many researchers including Wang, Lambert, Simpson, Mohapatra, Chaughry, Kassem, and Moloo, have focused on detecting partial blockages. Partial blockages can be detected by measuring the change in flow velocity caused by the reduced pipe cross-section where a partial blockage exists. This approach requires a pipeline to be instrumented throughout its length, or frequency analysis at the outlet $[28-30]$.

Little work has been published on detecting complete blockages where no flow reaches the outlet. Complete blockages can arise suddenly during operation due to debris entrainment, chemical reaction, improper pipeline operation, or changes in environmental conditions. In short single pipes a complete blockage's location can be estimated by measuring how long it takes for a transient pressure pulse to be reflected back to the inlet 
from the blockage [31]. This measured time of travel is then divided by the assumed constant wave speed in the single pipe and an estimate of the complete blockage location is obtained.

In long series pipelines the wave speed will not be consistent if the pipe diameter changes, the way the pipeline is anchored varies, or elbows are present $[1,2,32]$. This variable wave speed makes the time of travel method of detecting a complete blockage difficult.

\subsection{Unplugging of Complete Blockages}

Pipeline blockages can result from a number of different mechanisms: wax or solid hydrates can build up over time, pigs can become lodged in the lines, and pigging can also draw solids down the line to accumulate into a plug. Once a pipeline is blocked, production is lost and it becomes a matter of urgency to locate and remove the blockage [30].

Complex-wide, DOE has a need for a non-invasive method of clearing plugged radioactive waste transfer lines. A range of traditional techniques are currently used, despite their hazards. Over-pressurizing a line to attempt blockage removal is a common method, but this is often unsuccessful and undesirable. Other traditional invasive techniques include sewer snakes and water jetting. Since DOE waste transfer lines are usually buried, have few access connections, and contain radioactive material, inserting a snake or water jetting tool is not a good solution. While these methods can be effective, they create a significant problem with contamination cleanup and exposure of personnel to the pipe contents. In one case, a water jet hose lodged in the radioactive waste transfer 
line and had to be removed by dissolving it, which added a sticky, caustic substance to the system. Clearly a non-invasive technique is needed. Non-invasive methods would be much easier to use in highly contaminated systems, pose fewer problems, reduces risk to personnel, and reduce the risk of equipment lodging in the piping [98].

\subsection{Web Application for Engineering Software}

As the World Wide Web and its programming tools mature, we increasingly find analytical applications with Web interfaces and other Web sites with content generated instead of hand-created [33]. Most engineering software requires a specialized hardware and software environment to function. This is especially true for engineering software which takes advantage of multiple processing cores to perform analysis faster. The creation and maintenance of these specialized computing environments, commonly called clusters, is expensive and requires expertise. Consequently, at the time of writing there are relatively few computational clusters for scientific research when compared to the number of web servers or personal computers.

The United States is ranked in 29th place for consumer download speed. Results were obtained by analyzing test data between May 28, 2011 and Jun 26, 2011. Tests from 46,724,752 unique IPs have been taken in the United States and of 231,987,432 total tests, 7,457,005 are being used for the current Index [34]. The ability of high-speed internet to provide remote access to data has made it possible for users around the world to connect to cluster computers and for computing clusters to pool their resources to tackle large scale problems. Text base User Interfaces (TUI), have been the primary

mode of access over the internet to computing clusters. TUI are immensely capably but 
require users to be know a large set of commands in order to perform basic tasks involved with submitting jobs and monitoring them. Consequently the learning curve is steep and the interface is not viewed as user friendly.

Web pages provide a computationally efficient Graphical User Interface (GUI) to many web applications such as search engines, e-commerce store fronts, and online games. Harnessing the ability of web pages to provide a GUI interface for parallel computing applications is a logical step in the effort to develop high performance user friendly applications. 


\section{CHAPTER III}

\section{PHYSICAL PROBLEM AND MATHEMATICAL FORMULATION}

The safe and efficient operation of pipeline systems is a vital part of high level waste processing and many other industrial endeavors such as oil and natural gas refinement and distribution, water purification and distribution, and process cooling. In all of these applications the hydraulic fluid pressure generated at a remote location is sent through the pipeline to motivate the transport of the fluid to a facility for further processing or use or in a closed loop for heat removal. In all of these systems the pipelines may have highly complex configurations with numerous features such as branches, small radius $90^{\circ}$ elbows, surge tanks, reservoirs and valves.

The physical problem analyzed in this thesis is the effect of small radius $90^{\circ}$ on transients in series pipelines. The goal of the analysis is to determine the appropriate model and parameters for the damping effect of small radius $90^{\circ}$ elbows on pipeline transients. The loss parameters in the model are then determined through optimization with the objective of matching the peak pressure and transient profile from experimental data.

\subsection{Mathematical Formulation for Transient Flow in Closed Channel}

Hydraulic transient problems in pipeline systems have received increased attention in recent years, but few researchers have studied the effect of small radius $90^{\circ}$ elbows. No publications have been made on the application of optimization methods to transient modeling in pipelines with small radius $90^{\circ}$ elbows. The mathematical formulation herein described is based on transient flow in closed channel. 
Hydraulic transients occur when the steady-state conditions at a given point in the pipeline start changing with time, e.g., closing of a valve, failure of a pump, etc. This disturbance in the steady-state conditions causes a pressure wave will travel along the pipeline starting at the point of the disturbance. The pressure wave will be reflected back from the pipe boundaries (e.g., reservoirs) until a new steady-state is reached $[6,10,34$, 36].

Analysis of hydraulic transients in pressurized systems are carried out assuming quasi one-dimensional flow and are based on the continuity and momentum equations describing the general behavior of fluids in a closed channel.

In this chapter, the differential equations of continuity and momentum for transient flows are presented. The wave speed equation for thin-walled pipe is given and the effective bulk modulus, assuming presence of air/gas dispersed through the liquid, is discussed.

\subsubsection{Assumptions}

The following assumptions are made in the derivation of the equations:

- The flow is assumed to be one-dimensional $(x)$ and vary with respect to time

- The fluid is assumed to be compressible

- The average velocity and pressure distribution at a cross-section are assumed to be uniform on that cross-section

- Formulas for computing the steady-state friction losses in closed channels are valid during transient flows 


\subsubsection{Fluid Bulk Modulus}

The speed that a pressure wave propagates in a hydraulic line can only be determined by considering the compressibility of the fluid. A fluid's compressibility is quantified by its fluid bulk modulus.

The fluid bulk modulus describes the elasticity of the fluid at a certain temperature. This property is determined experimentally using a stress-strain test in which the volume of fluid is decreased while keeping the mass constant [37]. During this process, the stress of the fluid is measured by measuring the fluid pressure. A plot of the fluid pressure versus the fluid strain is then generated, and the slope of this plot is used to describe the elasticity of the fluid. This slope is generally referred to as the fluid bulk modulus.

The fluid bulk modulus is defined by the slope of a line that is anywhere tangent to the stress-strain curve, which is not linear. This quantity is expressed mathematically as

$$
K=\lim _{\Delta \varepsilon \rightarrow 0} \frac{\Delta p}{\Delta \varepsilon}=\frac{d p}{d \varepsilon}
$$

The non-dimensional fluid strain, $\varepsilon$, for the calculation of fluid bulk modulus is defined by

$$
\varepsilon \equiv-\ln \left(\frac{v}{v_{o}}\right)
$$

where $p$ is the fluid pressure, $v_{0}$ is the fluid volume at atmospheric pressure and $v$ is the volume at another point of interest.

Differentiating $\varepsilon \equiv-\ln \left(\frac{v}{v_{o}}\right)$

$(3-2)$ results in 


$$
d \varepsilon=-\frac{1}{V} d v
$$

Therefore, the fluid bulk modulus may be expressed as

$$
K=-v \frac{d p}{d v}
$$

Because mass is conserved

$$
m=\rho v
$$

Differentiation of both sides gives

$$
-\frac{1}{\rho} d \rho=\frac{1}{v} d v
$$

$1 \rho d \rho=1 v d v$

(3-6) into

$K=-v \frac{d p}{d v}$

(3-4) gives

$$
K=\rho \frac{d p}{d \rho}
$$

\subsubsection{Bulk Modulus for a liquid-air/gas mixture}

The fluid bulk modulus has been used to describe the elasticity of a fluid as it undergoes a volumetric deformation. This elasticity describes a spring like effect and the interaction of this restoring effect with the mass of mechanical parts gives rise to a resonance in nearly all hydraulic components.

The fluid bulk modulus can be substantially lowered by entrained air. Even a small amount of air can reduce the fluid bulk modulus by a factor of 10 . It is extremely important to know the resultant bulk modulus of the liquid and air/gas mixture because of its significant effect on the speed of propagation of pressure pulses in the mixture.

Let us consider a flexible pipe filled with a fluid which is a mixture of liquid and air/gas in form of bubbles. The total initial volume of the fluid, $v_{f}$, can be written as

$$
v_{f}=v_{l}+v_{g}
$$

where $v_{l}$ and $v_{g}$ are initial volumes of the liquid and gas, respectively. 
As a pressure increase $\Delta p$ is exerted on the fluid mixture, a change in the initial volume of fluid is observed and can be mathematically written as

$$
\Delta v_{f=} \Delta v_{l}+\Delta v_{g}
$$

The bulk modulus of the fluid (mixture of liquid and gas) can be defined, following the bulk modulus definition, as

$$
K_{f}=-v_{f} \frac{\Delta p}{\Delta v_{f}}
$$

or

$$
\frac{1}{K_{f}}=-\frac{\Delta v_{f}}{v_{f} \Delta p}
$$

$v f=v l+v g$

$v f=\Delta v l+\Delta v g$

$K f=-v_{f} \frac{\Delta p}{\Delta v_{f}}$

$1 K f=-\frac{\Delta v_{f}}{v_{f} \Delta p}$

$$
\frac{1}{K_{f}}=\frac{v_{l}}{v_{f}}\left(-\frac{\Delta v_{l}}{v_{l} \Delta p}\right)+\frac{v_{g}}{v_{f}}\left(-\frac{\Delta v}{v_{g} \Delta p}\right)
$$

Using the bulk modulus definition, $K=-v \frac{d p}{d v}$

(3-4), the liquid bulk modulus and the gas bulk modulus with respect to the their total initial volume, may be expressed, respectively, as

$$
K_{l}=-v_{l} \frac{\Delta p}{\Delta v_{l}}
$$

and

$$
K_{g}=-v_{g} \frac{\Delta p}{\Delta v_{g}}
$$


Substituting $K_{l}$, and $K_{g}$ into expression $1 K f=\frac{v_{l}}{v_{f}}\left(-\frac{\Delta v_{l}}{v_{l} \Delta p}\right)+\frac{v_{g}}{v_{f}}\left(-\frac{\Delta v}{v_{g} \Delta p}\right)$

\section{(3-12) gives}

$$
\frac{1}{K_{f}}=\frac{v_{l}}{v_{f}}\left(\frac{1}{K_{l}}\right)+\frac{v_{g}}{v_{f}}\left(\frac{1}{K_{g}}\right)
$$

This is a general equation which gives the bulk modulus for a liquid-gas mixture.

By introducing void fraction (relative volume of gas or vapor in the fluid mixture)

defined as $y_{g}=v_{g} / v_{f}$, this $1 K f=\frac{v_{l}}{v_{f}}\left(\frac{1}{K_{l}}\right)+\frac{v_{g}}{v_{f}}\left(\frac{1}{K_{g}}\right)$

\section{(3-15) becomes}

$$
\frac{1}{K_{f}}=\frac{1-y_{g}}{K_{l}}+\frac{y_{g}}{K_{g}}
$$

or

$$
K_{f}=\frac{K_{l}}{1+y_{g}\left(\frac{K_{l}}{K_{g}}-1\right)}
$$

\subsubsection{Wave Propagation Speed}

To introduce the concept of wavespeed or celerity, imagine firstly a case of instantaneous stoppage of flow at a downstream valve as described in Figure 3.1. Then the unsteady momentum and continuity equation is applied to a control volume containing a section of pipe Figure 3-1a. The instant the valve is closed, the fluid immediately adjacent to it is brought from $V_{0}$ to rest by the impulse of the higher pressure developed at the face of the valve. As soon as the first layer is brought to rest, the same action is applied to the next layer of fluid bringing it to rest. In this manner, pulse wave of high pressure is visualized as travelling upstream at some sonic wavespeed, $a$, and at sufficient pressure to apply just the impulse to the fluid to bring it to rest [1]. 
The momentum equation is applied to a control volume, Figure 3-1b, within which the wave front is moving to the left with an absolute speed of $a-V_{0}$ due to a small change in valve setting. The pressure change $\Delta p$ at the valve is accompanied by a velocity change $\Delta V$. The momentum equation for the $x$-direction states that the resultant $x$-component of force on the control volume is just equal to the time rate of increase of $x$-momentum within the control volume plus the net efflux of $x$-momentum from the control volume. The volume of fluid having its momentum changed is $A\left(a-V_{0}\right) \Delta t$, so the time rate of increase of linear momentum, neglecting friction and minor effects, is
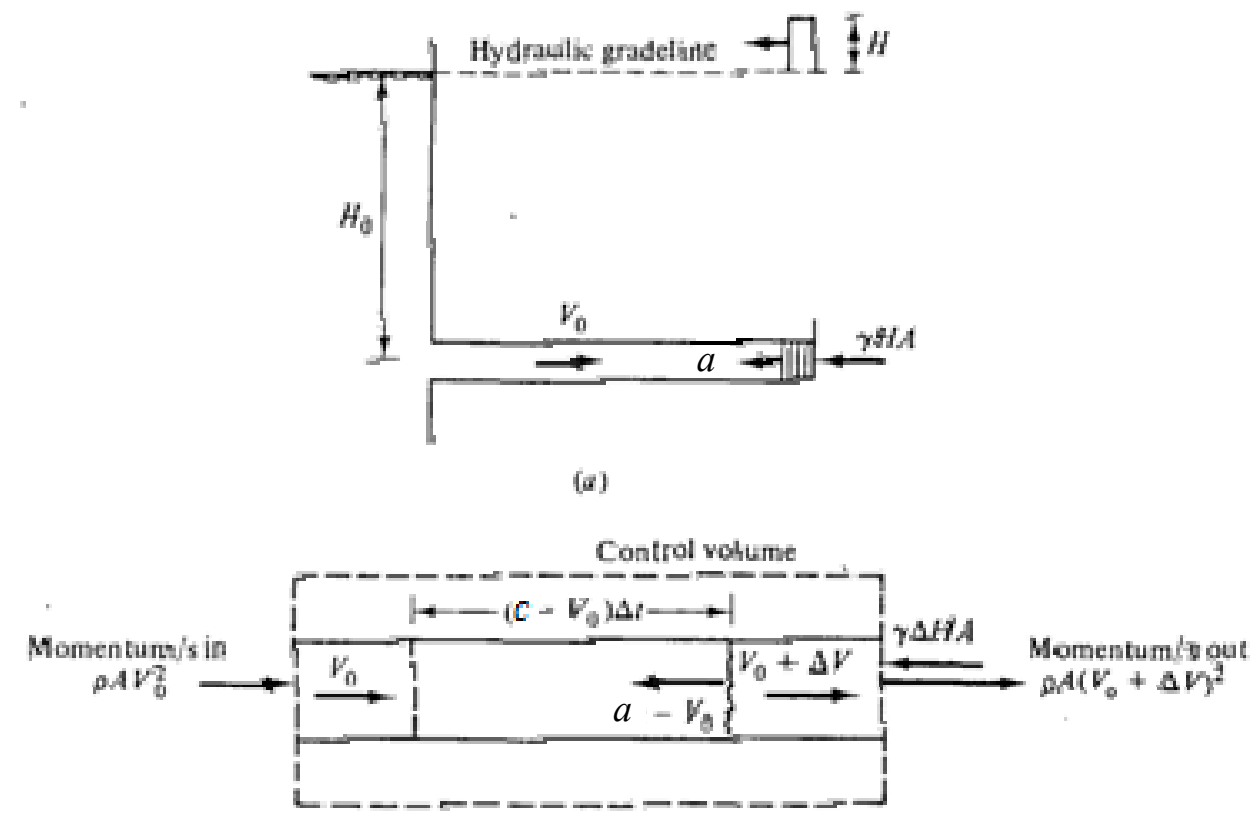

(b)

Figure 3-1: (a) Instantaneous stoppage of frictionless fluid in horizontal pipe; (b) Momentum equation applied to control volume [1].

$$
\rho A V_{0}-(\rho+\Delta \rho) A\left(V_{0}+\Delta V\right)=\frac{A\left(a-V_{0}\right) \Delta t[(\rho+\Delta \rho)-\rho]}{\Delta t}
$$


When simplified and combined with momentum equation in its simplified version, the following basic equation results

$$
\Delta p=-\rho a \Delta V
$$

Since $\Delta p=\rho g \Delta H$, in which $g$ is the acceleration of gravity, and $\Delta H$, the head change,

$\Delta H=-\frac{a \Delta V}{g}$

$$
\Delta p=-\rho a \Delta V
$$

If the flow is stopped completely $\Delta V=-V_{0}$ and $\Delta H=a V_{0} / g$. Equations

$\Delta p=-\rho a \Delta V$

(3-19) also

show that for an increase in velocity at the gate, the head there must be reduced. If the valve is on the downstream end of long pipe and is closed by increments, the equations become

$$
\sum \Delta p=-\rho a \sum \Delta V
$$

$\Delta H=-a g \Delta V$

$\Delta p=-\rho a \Delta V$

which holds for any movements of the valve as long as the pressure pulse wave has not reached the upstream end of the pipe and returned as a reflected wave, i.e., so long as the time is less than $2 L / a$, with $L$ the pipe length.

For adjustments in an upstream gate, a similar derivation shows that

$$
\Delta p=-\rho a \Delta V
$$

So

$$
\sum \Delta p= \pm \rho a \sum \Delta V
$$


describe the change in the flow related to a change in pressure. The minus sign must be used for waves traveling upstream and the plus sign for waves traveling downstream. It is the basic equation of waterhammer and always holds in the absence of reflections.

The magnitude of the wavespeed, $a$, has not been determined yet. Application of $\Delta p=-\rho a \Delta V$ (3-20), the numerical value of celerity, $a$, can be calculated. With reference to Figure 3-2, if the gate at the downstream end of the pipe is suddenly closed, the pipe may stretch in length $\Delta s$, depending on how it is supported [1].

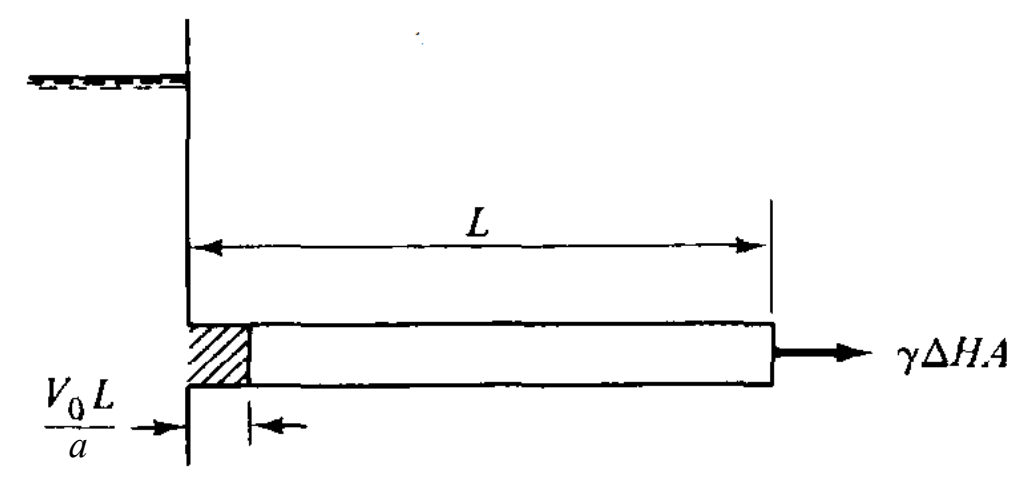

Figure 3-2: Continuity relations in pipe [1]

Assuming that the gate moves this distance in $L / a$ seconds, or has the speed $a \Delta s / L$, hence, the velocity of the fluid at the gate has been changed by $\Delta V=(a \Delta s / L)-V_{0}$. During $L / a$ seconds after gate closure, the mass entering the pipe is $\rho A V_{0} / a$, which is accommodated within the pipe by increasing its cross-sectional area, by 
fitting the extra volume due to pipe extension $\Delta s$, and by compressing the fluid due its higher pressure, or, representing mathematically

$$
\rho A V_{0} \frac{\mathrm{L}}{\mathrm{c}}=\rho \mathrm{L} \Delta \mathrm{A}+\rho \mathrm{A} \Delta \mathrm{s}+\mathrm{LA} \Delta \rho
$$

$V O L C=\rho L \Delta A+\rho A \Delta s+L A \Delta \rho$

(3-23) by substituting

into it $\Delta V=(a \Delta s / L)-V_{0}$

$$
-\frac{\Delta \mathbf{V}}{\mathbf{a}}=\frac{\Delta \mathbf{A}}{\mathbf{A}}+\frac{\Delta \boldsymbol{\rho}}{\boldsymbol{\rho}}
$$

By use of $\Delta p=-\rho a \Delta V$

(3-19a) in order to eliminate $\Delta \mathrm{V}$,

$$
\mathbf{a}^{2}=\frac{\frac{\Delta \mathbf{p}}{\boldsymbol{\rho}}}{\frac{\Delta \mathrm{A}}{\mathrm{A}}+\frac{\Delta \boldsymbol{\rho}}{\rho}}
$$

The equation is valid for pipe with or without expansion joints. The fluid bulk modulus definition may be used to rearrange, $K=\rho \frac{d p}{d \rho}$

\section{$\mathrm{a} 2=\Delta p \rho \Delta A A+\Delta \rho \rho$}

(3-25) to yield

$$
\mathbf{a}^{2}=\frac{\frac{\mathrm{K}_{\mathrm{f}}}{\rho_{\mathrm{f}}}}{1+\left(\frac{\mathrm{K}_{\mathrm{f}}}{\mathrm{A}}\right)\left(\frac{\Delta \mathrm{A}}{\Delta \mathrm{p}}\right)}
$$

The evaluation of the wave speed in a typical transient flow in thin-walled elastic pipeline requires knowledge of the fluid bulk modulus and density, and the evaluation of the pipe elasticity expressed by $\Delta A / A \Delta p$. According to Streeter and Wylie [1], this parameter is given by

$$
\frac{\Delta A}{A \Delta p}=\frac{D C_{1}}{E e}
$$


Here, $e$ is the wall thickness, $E$ is the modulus of elasticity of the wall material, $D$ is the inner diameter of the tube and $C_{1}$ is a non-dimensional parameter accounting for the degree of restraint applied to the system or hose. For a hose fully restrained along its whole length, $C_{1}=1$.

$A A \triangle p=D C 1 E e$

$\mathrm{a} 2=K f \rho f 1+K f A \Delta A \Delta p$

yields an equation that may be evaluated for a specific thin-walled elastic pipeline.

$$
a=\sqrt{\frac{\frac{K_{f}}{\rho_{f}}}{1+K_{f} \frac{D C_{1}}{E e}}}
$$

This equation represents the wave propagation speed, $c$, or celerity in a fluid mixture in a elastic pipeline.

$K f=K l 1+y g K l K g-1$

into $a=\sqrt{\frac{\frac{K_{f}}{\rho_{f}}}{1+K_{f} \frac{D C_{1}}{E e}}}$

writing fluid density in terms of liquid and gas density, represented respectively by $\rho_{l}$ and $\rho_{g}$ the celerity may be expressed in terms of bulk modulus of liquid and gas as follows

$$
a=\sqrt{\left.\frac{K_{l}}{\left[1+y_{g}\left(\frac{K_{l}}{K_{g}}-1\right)\right]\left(1-y_{g}\right) \rho_{l}+y_{g} \rho_{g}\left[1+K_{l} \frac{D C_{1}}{E e}\left[1+y_{g}\left(\frac{K_{l}}{K_{g}}-1\right)\right]\right.}\right]}
$$

If no gas/vapor is present in the fluid, then $y_{g}=0$, resulting in

$$
a=\sqrt{\frac{\frac{K_{l}}{\rho_{l}}}{1+K_{l} \frac{D C_{1}}{e E}}}
$$


Similarly, when the fluid is only gas or vapor (no liquid), the wave speed becomes

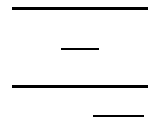

Notice that in both cases, the presence of the bulk modulus of the material of the tube wall is prominently displayed in the second term of the denominator.

\subsection{Equation of Motion}

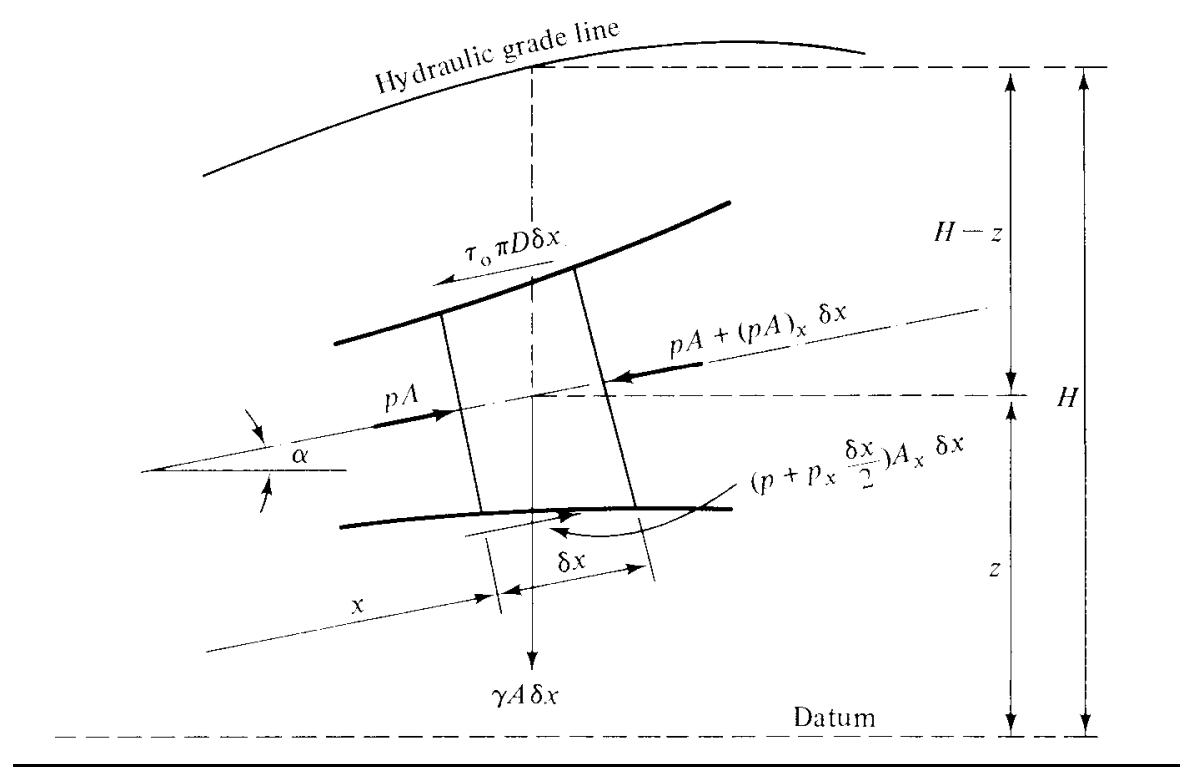

Figure 3-3: Free body diagram for application of the equation of motion [1]

Figure 3-3 above shows a free body diagram of a slice of fluid of cross sectional area $\mathrm{A}$ and thickness $\partial \mathrm{x}$. The area $\mathrm{A}$ is, in general, a function of $\mathrm{x}$, which is the coordinate distance along the axis of the tube from an arbitrary origin. The tube is inclined at an angle, $\alpha$, positive when the elevation increases in the $+x$ direction. The forces on the free body in the $\mathrm{x}$ direction are the surface contact normal pressures on the transverse faces, and shear and pressure components on the periphery. In addition 
gravity, the body force, has an $\mathrm{x}$ component. The shear force $\tau_{0}$ is considered to act in the $-\mathrm{x}$ direction. With reference to the figure the summation of forces on the slice of fluid is equated to its mass times its acceleration:

$$
\begin{aligned}
p A-\left[p A+(P A)_{x} \partial x\right] & +\left(p+p_{x} \frac{\partial x}{2}\right) A_{x} \partial x \\
& -\boldsymbol{\tau}_{\mathbf{0}} \boldsymbol{\pi} \boldsymbol{D} \boldsymbol{\partial} \boldsymbol{x}-\boldsymbol{\gamma} \boldsymbol{A} \boldsymbol{\partial} \boldsymbol{x \boldsymbol { s i n }}(\boldsymbol{\alpha})=\boldsymbol{\rho} \boldsymbol{A} \boldsymbol{\partial} \boldsymbol{x} \dot{\boldsymbol{V}}
\end{aligned}
$$

By dropping out the small quantity containing $(\partial x)^{2}$ and simplifying

$$
p_{x} A+\tau_{0} \pi D+\rho A \partial x \sin (\alpha)+\rho A \dot{V}=0
$$

In transient flow calculations the shear stress $\tau_{0}$ is considered to be the same as if the velocity was steady [1]. An expression for $\tau_{0}$ in terms of the Darcy-Weisbach friction factor, $\mathrm{f}$, can be developed by beginning with a force balance on the pipe in steady flow

$$
\Delta p \frac{\pi D^{2}}{4}=\tau_{0} \pi D L
$$

and the Darcy-Weisbach equation

$$
\Delta p=\frac{\rho f L}{D} \frac{V^{2}}{2}
$$

where $\mathrm{L}$ is the length of the horizontal pipe. Combining $\Delta p \frac{\pi D^{2}}{4}=\tau_{0} \pi D L$

$$
\text { (3-34) and } \Delta \boldsymbol{p}=\frac{\rho f L}{D} \frac{V^{2}}{2}
$$

\section{(3-35) produces}

$$
\tau_{0}=\frac{\rho V|V|}{8}
$$

$\tau 0=\rho V V 8$

that the shear stress always opposes the direction of the velocity [1].

$V$ in equation $p x A+\tau 0 \pi D+\rho A \partial x \sin \alpha+\rho A V=0$

is for the slice of fluid having a velocity $\mathrm{V}$, hence 


$$
\dot{V}=V V_{x}+V_{t}
$$

by use of equations $\Delta p \frac{\pi D^{2}}{4}=\tau_{0} \pi D L$

$=V V x+V t$

$p x A+\tau 0 \pi D+\rho A \partial x \sin \alpha+\rho A V=0$

(3-33) takes the form

$$
\frac{p_{x}}{\rho}+V V_{x}+V_{t}+g \sin (\alpha)+\frac{f V|V|}{2 D}=0
$$

which is valid for converging or diverging pipe flow as well [1]. The piezometric head $\mathrm{H}$ (or elevation of hydraulic grade line above an arbitrary datum) may replace $\mathrm{p}$.

From Figure 3-3:

$$
\boldsymbol{p}=\boldsymbol{\rho} \boldsymbol{g}(\boldsymbol{H}-\mathbf{z})
$$

where $\mathrm{z}$ is the elevation of the centerline of the pipe at $\mathrm{x}$. Then

$$
p_{x}=\rho g\left(H_{x}-z_{x}\right)=\rho g\left(H_{x}-\sin (\alpha)\right)
$$

The partial differential considered $\rho$ to be substantially constant, as compared

$p x p+V V x+V t+g \sin \alpha+f V V 2 D=0$

$p x=\rho g H x-z x=\rho g H x-\sin \alpha$

$p x p+V V x+V t+g \sin \alpha+f V V 2 D=0$

$$
g H_{x}+V V_{x}+V_{t}+\frac{f V|V|}{2 D}=0
$$

also restricted to liquid flow. The hydraulic grade line form of the equation is somewhat simpler, as the slope of the pipeline drops out [1].

\subsection{Continuity Equation}

In this section a derivation of the continuity equation developed by T. P. Propson (private communications) is presented. It is quite general and has the advantage of 
portraying the various total derivatives, i.e., derivatives with respect to the motion. Two come directly into the continuity equation; (1) differentiation with respect to the axial motion of the pipe, and (2) differentiation with respect to a particle of fluid mass. The third total derivative which arises from the characteristics method [1].

With reference to Figure 3-4 a moving control volume of length $\delta \mathrm{x}$ at time $t$ may be considered to be fixed relative o the pipe-it moves and stretches only as the inside surface of the pipe moves and stretches. The conservation of mass law may be stated that the time rate of mass inflow into this control volume is just equal to the time rate of increase of mass within the control volume, or

$$
-[\rho A(V-u)]_{x} \delta x=\frac{D \prime}{D t}(\rho A \delta x)
$$

Let the upstream face be at $\mathrm{x}$, and is the velocity of the pipe wall at $\mathrm{x}$. The total derivative with respect to the axial motion of the pipe is given by

$$
\frac{D^{\prime}}{D t}=u \frac{\partial}{\partial x}+\frac{\partial}{\partial t}
$$

and the time rate of increase of length $\delta x$ of the control volume is given by

$$
\begin{gathered}
\frac{D^{\prime}}{D t} \delta x=u_{x} \delta x \\
\rho A V-u x \delta x=D^{\prime} D t \rho A \delta x
\end{gathered}
$$

(3-42) with use of

'Dt $\delta x=u x \delta x$

$$
(\rho A V)_{x}+(\rho A u)_{x}+\frac{D^{\prime}}{D t}(\rho A)=0
$$




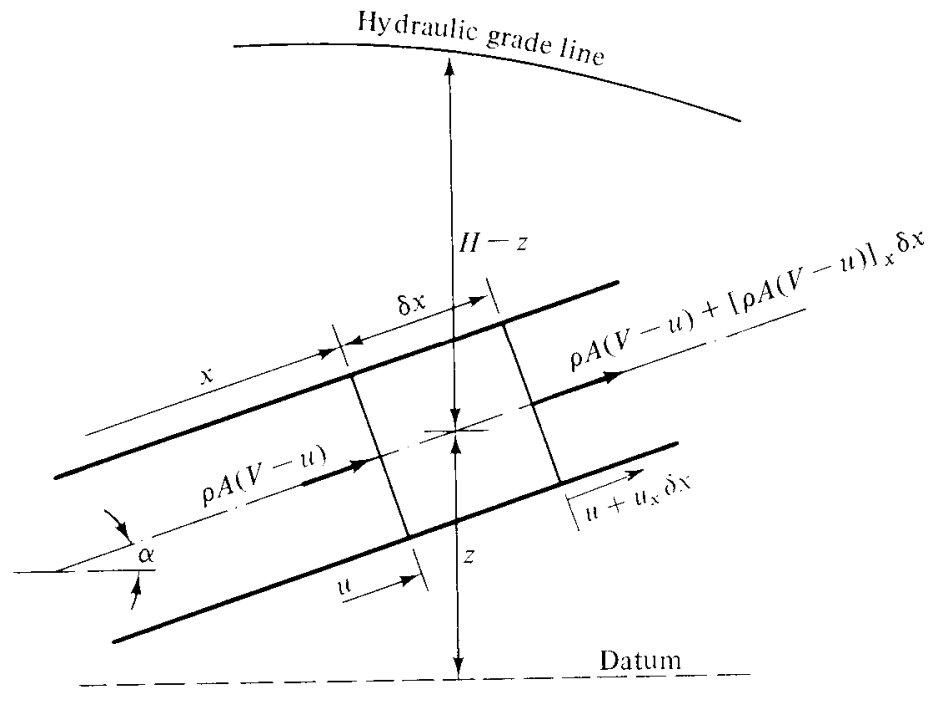

Figure 3-4: Control volume for continuity

$$
\begin{aligned}
& +{ }^{\prime}=0 \\
& =\quad+ \\
& \text { yields }
\end{aligned}
$$$$
\text { (3-45), using equation }
$$

or by simplifying

which now may be written as

The last two terms represent the derivative of $\rho \mathrm{A}$ with respect to the motion of a mass particle, or

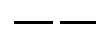

in which

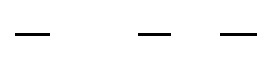

This total derivative is also indicated by a dot over the dependant variable, so 


$$
\frac{1}{\rho A} \frac{D}{D t}(\rho \dot{A}+\dot{\rho} A)+V_{x}=V \frac{\partial}{\partial t}+\frac{\partial}{\partial x}
$$

or

$$
\frac{\dot{A}}{A}+\frac{\dot{\rho}}{\rho}+V_{x}=0
$$

It is informative to introduce the effect of Poisson's ratio on wave-speeds for the

$A A+\rho \rho+V x=0$

$$
\frac{\dot{p}}{K}=\frac{\dot{\rho}}{\rho}
$$

where $\mathrm{K}$ is the bulk modulus of elasticity of the fluid.

$$
K=\frac{\Delta p}{\Delta \rho / \rho}=-\frac{\Delta p}{\Delta V / V}
$$

The pipe wall expansion per unit area per unit time $\dot{A} / A$ is

$$
\dot{A}=2 \dot{\xi_{T}}
$$

Where from the Poisson's ratio relations

$$
\dot{\xi_{T}}=\frac{1}{E}\left(\dot{\sigma_{2}}-\mu \dot{\sigma_{1}}\right)
$$

$p K=\rho \rho$

REF_Ref296427277 $\backslash \mathrm{h} \backslash *$ MERGEFORMAT $K=\frac{\Delta p}{\Delta \rho / \rho}=-\frac{\Delta p}{\Delta V / V}$

$A A=2 \xi T$

(3-55) in

$A A+\rho \rho+V x=0$

$\frac{2}{E}\left(\dot{\sigma}_{2}-\mu \dot{\sigma_{1}}\right)+\frac{\dot{p}}{K}+V_{x}=0$

The transverse, or circumferential, tension is related to pressure by

$$
\sigma_{2}=p D /(2 e)
$$

or

$$
\dot{\sigma_{2}}=\dot{p} D /(2 e)
$$


D changes so little with time, as compared to $p$ in transient flow that it is considered constant for this differentiation. The axial rate of chance of the tensile stress $\dot{\sigma}_{1}$ is given for the case of a pipeline anchored throughout [1].

$$
\dot{\sigma_{2}}=\mu \dot{\sigma_{1}}
$$

Equation (3-51) through substitution of equations (3-52) and (3-53) may be written

$$
\frac{\dot{\rho}}{\rho}+a^{2} V_{x}=0
$$

where $\mathrm{a}$ is a constant that is a collection of properties if the fluid, the pipe, and its means of support, and so far has been given no meaning relating it to acoustic speed.

$$
a^{2}=\frac{K / \rho}{1+[(K / E)(D / e)] c_{1}}
$$

and

$$
c_{1}=1-\mu^{2}
$$

for a pipe line anchored throughout.

The piezometric head may be introduced into equation (3-54); from Figure 3-4

$$
p=\rho g(H-z)
$$

and

$$
\dot{p}=\rho g(\dot{H}-\dot{z})=\rho g\left(V H_{x}+H_{t}-V z_{x}-z_{t}\right)
$$

If the pipe has no transverse motion, $z_{t}=0$, and $z_{x}=\sin (\alpha)$ then equation (3-54) becomes

$$
V H_{x}+H_{t}-V \sin (\alpha)+\frac{a^{2}}{g} V_{x}=0
$$

which is a convenient form of the continuity equation with $\mathrm{V}$ and $\mathrm{H}$ as dependent variables, and with $\mathrm{x}$ and $\mathrm{t}$ the dependent variables. Through $\mathrm{a}^{2}$ the fluid and wall properties are included. 


\section{CHAPTER IV}

\section{METHOD OF SOLUTION}

In chapter 3 , the equations of motion and continuity that govern the unsteady fluid flow in closed channels were presented. These equations form a system of quasi-linear, hyperbolic partial differential equations in terms of two dependent variables, average velocity $V(x, t)$ and pressure $p(x, t)$ or hydraulic-grade line elevation $H(x, t)$, and two independent variables, distance along pipe length $x$, and time $t$.

Knowing that a general solution for a system of hyperbolic partial differential equations is not available, the employment of numerical methods is necessary. Some methodologies such as the graphical method, the impedance method, the method of characteristics may be used in order to approximate the solution. The choice of one of these methods is based on the restrictive assumptions that each one employs and if such assumptions can be applied in the model.

The method of characteristics is the most used when it is desirable to approximate the solution of a hydraulic transient problem in pipes because, according to Streeter [1], it has the best accuracy of any of the other methods. Another advantage is that this method does not neglect the non-linear terms of partial differential equations.

Details of the method of characteristics will be presented in this chapter. The system of partial differential equations will be transformed to ordinary differential equations, so that, those new equations may be later integrated to yield finite difference equations, which are conveniently handled numerically. The chapter concludes with the developing of boundary conditions for a number of simple inlet and outlet conditions. 


\subsection{Method of Characteristics}

The method of characteristics converts the two partial differential equations of motion and continuity into four ordinary differential equations. These equations are then expressed in finite difference form, using the method of specified time intervals, and solutions are carried out with use of computers [1].

The numerical solution is driven through characteristics equations, $C^{+}$and $C^{-}$, mapped in the Cartesian coordinate system $(x, t)$ and the chosen time interval $\Delta t$, and number of divisions $N+1$, are interconnected by the following relation:

$$
\Delta t=\frac{\Delta x}{c}
$$

where $c$ is the wave speed propagation through the pipe.

For the time increment, it is used a method of specified interval which maps the Cartesian coordinate system $(x, t)$ in a fixed mesh and, hence, a solution ordered in time and space $(x)$ is obtained.

Figure 4-1 and Figure 4-2, respectively, show the characteristic equations and the solution in a Cartesian coordinate system $(x, t)$.

In this presented work, the transient propagation speed was considered constant, which restricts the characteristics curves to straight lines.

From Figure 4-1, knowing the variables $V(x, t)$ and $p(x, t)$ or $H(x, t)$ at points A and B for a given time interval, it is possible to integrate simultaneously the equation valid through $C^{+}$between $\mathrm{A}$ and $\mathrm{P}$ and the equation valid through $C^{-}$between $\mathrm{B}$ and $\mathrm{P}$ and the result gives the value for $V(x, t)$ and $p(x, t)$ or $H(x, t)$ at point $\mathrm{P}$ for subsequent time [1]. 


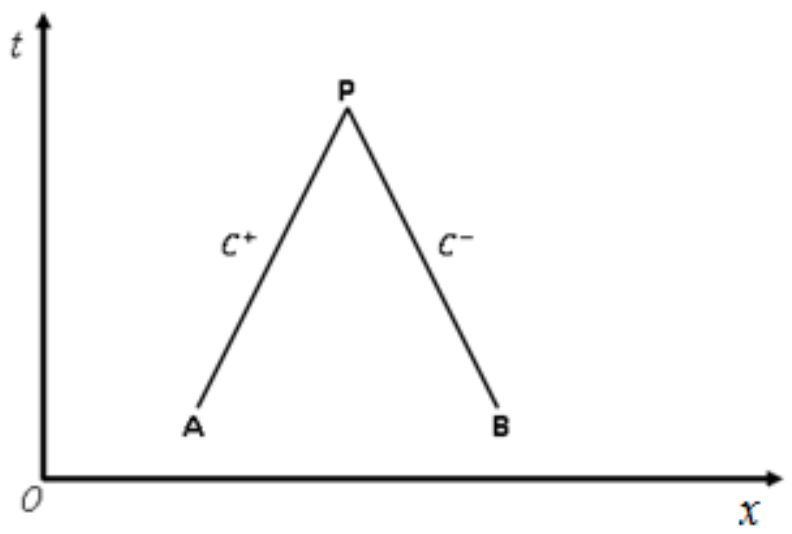

Figure 4-1: Characteristics curves in the Cartesian coordinates system [1]

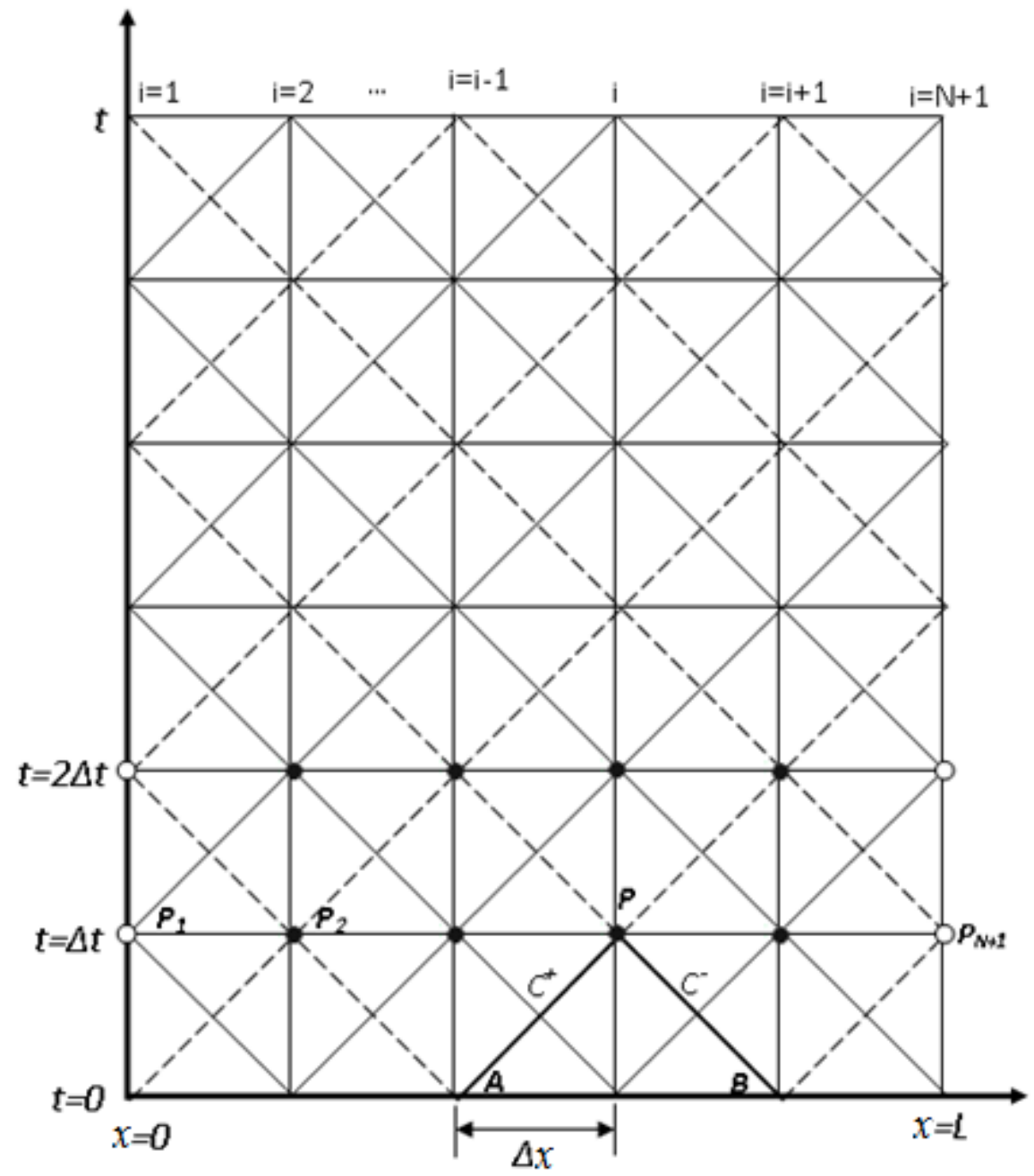

Figure 4-2: $(x, t)$ grid for solving single pipe problem [1] 


\subsubsection{Characteristics equations}

The momentum and continuity equations for a pair of quasi-linear hyperbolic partial differential equations in terms of two dependant variables, velocity, $\mathrm{V}$, and hydraulic grade line elevation, $\mathrm{H}_{\mathrm{x}}$, and two independent variables, distance along the pipe, $\mathrm{x}$, and time, $\mathrm{t}$.

The major terms of the momentum (3-39) and continuity (3-57) equations are transformed by the method of characteristics into equations (4-2) and (4-3) [1]

$$
\begin{aligned}
& L_{1}=g H_{x}+V_{t}+\frac{f}{2 D} V|V|=0 \\
& L_{2}=H_{t}+\frac{a^{2}}{g} V_{x}=0
\end{aligned}
$$

These equations are presented for a straight featureless pipeline. The presence of $90^{\circ}$ elbows in a pipeline effects both the velocity and the hydraulic grade line elevation along the pipeline.

I propose adding a loss term based on the geometry of a pipeline with $90^{\circ}$ elbows to the equation of motion (4-2). In order to develop the loss term the lengths of the pipe sections between the elbows must be known.

$$
G_{j}=\frac{c_{g}}{s_{j}}
$$

where $s_{j}$ represents the length of the $\mathrm{j}^{\text {th }}$ pipe section between elbows of the $\mathrm{N}$ sections present in the test bed and $c_{g}$ is the coefficient of geometric impedance. $c_{g}$ is a parameter to be optimized along with the friction factor for the overall pipeline, f. $L_{1}$ can be redefined by including the geometric loss term as

$$
L_{1}=g H_{x}+V_{t}+\frac{f}{2 D} V|V|+\frac{c_{g}}{s_{j}}=0
$$


These equations are combined linearly using an unknown multiplier $\lambda$.

$$
L=L_{1}+\lambda L_{1}=\lambda\left[H_{x} \frac{g}{\lambda}+H_{t}\right]+\left[V_{x} \lambda \frac{a^{2}}{g}+V_{t}\right]+\frac{f}{2 D} V|V|+\frac{c_{g}}{s_{j}}=0
$$

Any two real, distinct values of $\lambda$ will again yield two equations in terms of two dependent variables $\mathrm{H}$ and $\mathrm{V}$ that are in every way equivalent to equations $\left(4-2^{*}\right)$ and (4-3*). Appropriate selection of the two particular values of $\lambda$ leads to simplification of equation (4-3*). In general variables $\mathrm{V}$ and $\mathrm{H}$ are functions of $\mathrm{x}$ and $\mathrm{t}$. If the independent variable $\mathrm{x}$ is permitted to be a function of $\mathrm{t}$, then from calculus

$$
\frac{d H}{d t}=H_{x} \frac{d x}{d t}+H_{t} \quad \frac{d V}{d t}=V_{x} \frac{d x}{d t}+V_{t}
$$

Now, by examination of equation (4-3*) with equation (4-4) in mind, in can be noted that if

$$
\frac{d x}{d t}=\frac{g}{\lambda}=\frac{\lambda a^{2}}{g}
$$

Equation (4-4*) becomes the ordinary differential equation

$$
\lambda \frac{d H}{d t}+\frac{d V}{d t}+\frac{f}{2 D} V|V|+\frac{c_{g}}{s_{j}}=0
$$

The solution of equation (4-5) yields the two particular values of $\lambda$ :

$$
\lambda= \pm \frac{g}{a}
$$

By substituting these values back into equation (4-6), the particular manner in which $\mathrm{x}$ and $\mathrm{t}$ are related is given,

$$
\frac{d x}{d t}= \pm a
$$

This show the change in position of a wave related to the change in time by the wave propagation velocity a. When the positive value of $\lambda$ is used in equation (4-5), the positive value of $\lambda$ must be used in equation (4-6). A similar parallelism exists for the 
negative $\lambda$. The substitution of these values into equation (4-7) leads to two pairs of equations which are grouped and identified as $\mathrm{C}^{+}$and $\mathrm{C}^{-}$equations [1].

$\mathrm{C}^{+}$:

$$
\begin{aligned}
& \frac{g}{a} \frac{d H}{d t}+\frac{d V}{d t}+\frac{f}{2 D} V|V|+\frac{c_{g}}{s_{j}}=0 \\
& \frac{d x}{d t}=+a
\end{aligned}
$$

$\mathrm{C}-$ :

$$
\begin{aligned}
& -\frac{g}{a} \frac{d H}{d t}+\frac{d V}{d t}+\frac{f}{2 D} V|V|+\frac{c_{g}}{s_{j}}=0 \\
& \frac{d x}{d t}=-a
\end{aligned}
$$

Thus the two real values of $\lambda$ have been used to convert the original two partial differential equations into two total differentia equations, $\left(4-9^{*}\right)$ and $\left(4-11^{*}\right)$, each with the restriction that it is only valid when the respective equations (4-10) and (4-12) are valid.

No mathematical approximations have been made in this transformation of the original partial differential equations. Thus, every solution of this set will be a solution of the original system given by equations (4-2) and (4-3).

\subsection{Finite-difference equations}

A pipeline is divided into $N$ equal sections, each $\Delta x$ in length as shown in Figure 4-2. A time-step size is computed, $d t=\left(\frac{1}{V+a}\right) d x$, and (4-10) is satisfied by a positively sloped diagonal of the grid, shown by the line $\overline{A P}$. If the dependent variables $V(x, t)$ and $H(x, t)$ are known at $A$, then (4-9), which is valid along $C^{+}$line, can be integrated between the limits $A$ and $P$ and thereby be written in terms of unknown variables $V(x, t)$ 
and $H(x, t)$ at point $P$. Equation (4-11*) is satisfied on negatively sloped diagonal of the grid, shown by the line $\overline{B P}$. Integration of the $C^{-}$compatibility equation along the line $\overline{B P}$, for which conditions are known at $B$ and unknown at $P$, leads to a second equation in terms of the same two unknown variables at $P$. A simultaneous solution yields conditions at the particular time and position in the $x t$-plane designated by point $P$.

In order to represent (4-9*) and (4-11*) in a finite-difference form, some assumptions must be made:

- Pipe slope is assumed constant in a given section;

- Friction factor is assumed constant, as explained in the previous section; and

The integration of the positive compatibility and characteristic equations, respectively (4-9*) and (4-10), yields

$$
\begin{aligned}
\left(V_{P}-V_{A}\right)+\frac{g}{a}\left(H_{P}-H_{A}\right)-\frac{g}{a}\left(\frac{V_{P}+V_{A}}{2}\right) \sin \alpha_{A P}\left(t_{P}-t_{A}\right) \\
+\frac{f\left(t_{P}-t_{A}\right)}{2 D} V_{P}\left|V_{A}\right|+\frac{c_{g}}{s_{j}}=0 \\
\left(x_{P}-x_{A}\right)=\left(V_{A}+a\right)\left(t_{P}-t_{A}\right)
\end{aligned}
$$

A similar integration of the $C^{-}$equations, (4-11*) and (4-13b), provides a set of equations that can be solved numerically

$$
\begin{aligned}
&\left(V_{P}-V_{B}\right)-\frac{g}{a}\left(H_{P}-H_{B}\right)+ \frac{g}{a}\left(\frac{V_{P}+V_{B}}{2}\right) \sin \alpha_{B P}\left(t_{P}-t_{B}\right) \\
&+\frac{f\left(t_{P}-t_{B}\right)}{2 D} V_{P}\left|V_{B}\right|+\frac{c_{g}}{s_{j}}=0 \\
&\left(x_{P}-x_{B}\right)=\left(V_{B}-a\right)\left(t_{P}-t_{B}\right)
\end{aligned}
$$

Substituting $V=Q / A$, where $Q$ is volumetric flow rate, back into eqs. (4-13a) and (4-14a), gives 


$$
\begin{aligned}
\frac{a}{g A}\left(Q_{P}-Q_{A}\right)+\left(H_{P}-H_{A}\right)- & \left(\frac{Q_{P}+Q_{A}}{2 A}\right) \sin \alpha_{A P}\left(t_{P}-t_{A}\right) \\
& +\frac{f\left(t_{P}-t_{A}\right)}{2 D g A^{2}} Q_{P}\left|Q_{A}\right|+\frac{c_{g}}{s_{j}}=0 \\
\frac{a}{g A}\left(Q_{P}-Q_{B}\right)-\left(H_{P}-H_{B}\right)+ & \left(\frac{Q_{P}+Q_{B}}{2 A}\right) \sin \alpha_{B P}\left(t_{P}-t_{B}\right) \\
& +\frac{f\left(t_{P}-t_{B}\right)}{2 D g A^{2}} Q_{P}\left|Q_{B}\right|+\frac{c_{g}}{s_{j}}=0
\end{aligned}
$$

Equations (4-15) and (4-16) are basic algebraic relations that describe the transient propagation of flow and piezometric head in a pipeline [1].

Substituting eqs.(4-13b) and (4-14b) into (4-15) and (4-16), respectively, results

$$
\begin{gathered}
\frac{a}{g A}\left(Q_{P}-Q_{A}\right)+\left(H_{P}-H_{A}\right)-\left(\frac{Q_{P}+Q_{A}}{2 A\left(\frac{Q_{A}}{A}+a\right)}\right) \sin \alpha_{A P}\left(x_{P}-x_{A}\right)+ \\
\frac{f\left(x_{P}-x_{A}\right)}{2 D g A^{2}\left(\frac{Q_{A}}{A}+a\right)} Q_{P}\left|Q_{A}\right|+\frac{c_{g}}{s_{j}}=0 \\
\frac{a}{g A}\left(Q_{P}-Q_{B}\right)-\left(H_{P}-H_{B}\right)+\left(\frac{Q_{P}+Q_{B}}{2 A\left(\frac{Q_{B}}{A}-a\right)}\right) \sin \alpha_{B P}\left(x_{P}-x_{B}\right)+ \\
\frac{f\left(x_{P}-x_{B}\right)}{2 D g A^{2}\left(\frac{Q_{B}}{A}+a\right)} Q_{P}\left|Q_{B}\right|+\frac{c_{g}}{s_{j}}=0
\end{gathered}
$$

In (4-17), since $z_{P}$ and $z_{A}$ are the vertical heights in relation to the reference line established to the pipe center line in Figure 3-4, then

$$
\sin \alpha_{A P}=\frac{z_{P}-z_{A}}{\sqrt{\left(x_{P}-x_{A}\right)^{2}+\left(y_{P}-y_{A}\right)^{2}+\left(z-z_{A}\right)^{2}}}
$$

Plugging (4-19) into (4-17), valid along $C^{+}$, gives

$$
\frac{a}{g A}\left(Q_{P}-Q_{A}\right)+\left(H_{P}-H_{A}\right)-\left(\frac{Q_{P}+Q_{A}}{2 A\left(\frac{Q_{A}}{A}+a\right)}\right) \frac{\left(z_{P}-z_{A}\right)\left(x_{P}-x_{B}\right)}{\sqrt{\left(x_{P}-x_{A}\right)^{2}+\left(y_{P}-y_{A}\right)^{2}+\left(z-z_{A}\right)^{2}}}
$$




$$
+\frac{f\left(x_{P}-x_{A}\right)}{2 D g A^{2}\left(\frac{Q_{A}}{A}+a\right)} Q_{P}\left|Q_{A}\right|+\frac{c_{g}}{s_{j}}=0
$$

Since the same is valid for $C^{-},(4-18)$ becomes

$$
\begin{gathered}
\frac{a}{g A}\left(Q_{P}-Q_{B}\right)-\left(H_{P}-H_{B}\right)-\left(\frac{Q_{P}+Q_{B}}{2 A\left(\frac{Q_{B}}{A}-a\right)}\right) \frac{\left(z_{P}-z_{A}\right)\left(x_{P}-x_{B}\right)}{\sqrt{\left(x_{P}-x_{A}\right)^{2}+\left(y_{P}-y_{A}\right)^{2}+\left(z-z_{A}\right)^{2}}} \\
+\frac{f\left(x_{P}-x_{B}\right)}{2 D g A^{2}\left(\frac{Q_{B}}{A}-a\right)} Q_{P}\left|Q_{B}\right|+\frac{c_{g}}{s_{j}}=0
\end{gathered}
$$

For a rectangular grid,

- $\quad$ Characteristic curve valid along $C^{+}$

$$
\begin{aligned}
\frac{a}{g A}\left(Q_{P}-Q_{A}\right)+\left(H_{P}\right. & \left.-H_{A}\right)-\left(\frac{Q_{P}+Q_{A}}{2 A\left(\frac{Q_{A}}{A}+a\right)}\right) \frac{\Delta x \Delta z}{\sqrt{\Delta x^{2}+\Delta y^{2}+\Delta z^{2}}} \\
& +\frac{f \Delta x}{2 D g A^{2}\left(\frac{Q_{A}}{A}+a\right)} Q_{P}\left|Q_{A}\right|+\frac{c_{g}}{s_{j}}=0
\end{aligned}
$$

- $\quad$ Characteristic curve valid along $C^{-}$

$$
\begin{array}{r}
\frac{a}{g A}\left(Q_{P}-Q_{B}\right)-\left(H_{P}-H_{B}\right)-\left(\frac{Q_{P}+Q_{B}}{2 A\left(\frac{Q_{B}}{A}-a\right)}\right) \frac{\Delta x \Delta z}{\sqrt{\Delta x^{2}+\Delta y^{2}+\Delta z^{2}}} \\
+\frac{f \Delta x}{2 D g A^{2}\left(\frac{Q_{B}}{A}-a\right)} Q_{P}\left|Q_{B}\right|+\frac{c_{g}}{s_{j}}=0
\end{array}
$$

By solving eqs.(4-22) and (4-23) for $H_{P}$

$$
\begin{array}{r}
H_{P}=H_{A}-\frac{a}{g A}\left(Q_{P}-Q_{A}\right)+\frac{\Delta x \Delta z}{2 A \sqrt{\Delta x^{2}+\Delta y^{2}+\Delta z^{2}}} \frac{\left(Q_{P}+Q_{A}\right)}{\left(\frac{Q_{A}}{A}+a\right)} \\
-\frac{f \Delta x}{2 D g A^{2}} \frac{Q_{P}\left|Q_{A}\right|}{\left(\frac{Q_{A}}{A}+a\right)}+\frac{c_{g}}{s_{j}} \\
H_{P}=H_{B}+\frac{a}{g A}\left(Q_{P}-Q_{B}\right)-\frac{\Delta x \Delta z}{2 A \sqrt{\Delta x^{2}+\Delta y^{2}+\Delta z^{2}}} \frac{\left(Q_{P}+Q_{B}\right)}{\left(\frac{Q_{B}}{A}-a\right)} \\
+\frac{f \Delta x}{2 D g A^{2}} \frac{Q_{P}\left|Q_{B}\right|}{\left(\frac{Q_{B}}{A}-a\right)}+\frac{c_{g}}{s_{j}}
\end{array}
$$

Calling 


$$
\begin{aligned}
& B=\frac{a}{g A} \\
& S=\frac{\Delta x \Delta z}{2 A \sqrt{\Delta x^{2}+\Delta y^{2}+\Delta z^{2}}} \\
& R=\frac{f \Delta x}{2 D g A^{2}}
\end{aligned}
$$

Hence

$$
\begin{aligned}
& H_{P}=H_{A}-B\left(Q_{P}-Q_{A}\right)+S \frac{\left(Q_{P}+Q_{A}\right)}{\left(\frac{Q_{A}}{A}+a\right)}-R \frac{Q_{P}\left|Q_{A}\right|}{\left(\frac{Q_{A}}{A}+a\right)}+\frac{c_{g}}{s_{j}} \\
& H_{P}=H_{B}+B\left(Q_{P}-Q_{B}\right)-\mathrm{S} \frac{\left(Q_{P}+Q_{B}\right)}{\left(\frac{Q_{B}}{A}-a\right)}+R \frac{Q_{P}\left|Q_{B}\right|}{\left(\frac{Q_{B}}{A}-a\right)}+\frac{c_{g}}{s_{j}}
\end{aligned}
$$

Rearranging equations above

$$
\begin{gathered}
H_{P}=\left[H_{A}+B Q_{A}+S \frac{Q_{A}}{\left(\frac{Q_{A}}{A}+a\right)}+F \frac{Q_{A}}{\left(\frac{Q_{A}}{A}+a\right)}\right] \\
-\left[B-\frac{1}{\left(\frac{Q_{A}}{A}+a\right)}\left(S-R\left|Q_{A}\right|-2 F\right)\right] Q_{P}+\frac{c_{g}}{s_{j}} \\
H_{P}=\left[H_{B}-B Q_{B}-S \frac{Q_{B}}{\left(\frac{Q_{B}}{A}-a\right)}-F \frac{Q_{B}}{\left(\frac{Q_{B}}{A}-a\right)}\right] \\
+\left[B+\frac{1}{\left(\frac{Q_{B}}{A}-a\right)}\left(-S+R\left|Q_{B}\right|\right)+2 \mathrm{~F}\right] Q_{P}+\frac{c_{g}}{s_{j}}
\end{gathered}
$$

The solution to a problem in fluid transients usually begins with steady-state conditions at time zero, so that $Q, H$ are known initial values at each section of Figure 3-3, for $t=0$. The solution consists of finding $Q, H$, for alternate grid point along $t=\Delta t$, then proceding to $t=2 \Delta t$, and so on, until the desired time duration has been covered. At any interior grid intersection point, point $P$ at section $i$, the two compatibility equations are solved simultaneously for the unknows $Q_{i}{ }^{t+1}, H_{i}{ }^{t+1}$. Equations (4-31) and (4-32) may be written in a simple form, namely 


$$
\begin{aligned}
& H_{i}{ }^{t+1}=C_{P}{ }^{t}-B_{P}{ }^{t} Q_{i}{ }^{t+1}+\frac{c_{g}}{s_{j}} \\
& H_{i}{ }^{t+1}=C_{M}{ }^{t}+B_{M}{ }^{t} Q_{i}{ }^{t+1}+\frac{c_{g}}{s_{j}}
\end{aligned}
$$

in which the coefficients $C_{P}{ }^{t}, B_{P}{ }^{t}, C_{M}{ }^{t}$ and $B_{M}{ }^{t}$ are known constants when the equations are applied. Their values in the $C^{+}$and $C^{-}$compatibility equations are, respectively

$$
\begin{aligned}
C_{P}{ }^{t} & =\left[H_{i-1}^{t}+B Q_{i-1}^{t}+S \frac{Q_{i-1}^{t}}{\left(\frac{Q_{i-1}^{t}+a}{A}+a\right.}+\frac{c_{g}}{s_{j}}\right] \\
B_{P}{ }^{t} & =\left[B-\frac{1}{\left(\frac{Q_{i-1}^{t}}{A}+a\right)}\left(S-R\left|Q_{i-1}^{t}\right|+\frac{c_{g}}{s_{j}}\right)\right] \\
C_{M}{ }^{t} & =\left[H_{i+1}^{t}-B Q_{i+1}^{t}-S \frac{Q_{i+1}^{t}}{\left(\frac{\left.Q_{i+1}^{t}-a\right)}{A}-+\frac{c_{g}}{s_{j}}\right]}\right. \\
B_{M}{ }^{t} & =\left[B+\frac{1}{\left(\frac{Q_{i+1}^{t}}{A}-a\right)}\left(-S+R\left|Q_{i+1}^{t}\right|\right)++\frac{c_{g}}{s_{j}}\right]
\end{aligned}
$$

By first eliminating $Q_{i}$ in eqs.(4-33) and (4-34), the value for $H_{i}^{t+1}$

$$
H_{i}^{t+1}=\left(\frac{C_{P} B_{M}+C_{M} B_{P}}{B_{P}+B_{M}}\right)^{t}+\frac{c_{g}}{s_{j}}
$$

Then $Q_{i}$ may be found by substituting (4-39) directly into (4-33) or (4-34). Hence

$$
Q_{i}^{t+1}=\left(\frac{C_{P}-C_{M}}{B_{P}+B_{M}}\right)^{t}+\frac{c_{g}}{s_{j}}
$$

Numerical values of $H$ and $Q$ are found at alternate grid intersection points as shown in Figure 3-2. Examination of this grid shows that the end points of the system begin influencing the interior points after the first time step. Therefore, in order to 
complete the solution to any desired time, it is necessary to introduce the appropriate boundary conditions [1].

\subsubsection{Boundary conditions}

At either end of a single pipe only one of the compatibility equations is available in the two variables. For the upstream end (Figure 4-3a), (4-34) holds along the $C^{-}$ characteristic, and for the downstream bounday (Figure 4-3b), (4-33) is valid along the $C^{+}$characteristic. These are linear equations in $Q_{i}{ }^{t+1}$ and $H_{i}{ }^{t+1}$; each conveys to its respective boundary the complete behavior and response of the fluid in the pipeline

during the transient. An auxiliary equation is needed in each case that specifies $Q_{i}{ }^{t+1}$, $H_{i}{ }^{t+1}$ or some relation between them. That is, the auxiliary equation must convey information on the behavior of the boundary to the pipeline. This may be just the end condition of the pipeline, or it may be a different element or facility attached to the end of the pipe. Each boundary condition is solved independent of the other boundary, and independently of the interior point calculations [1] by satisfying the appropriate one of the characteristic equations. 


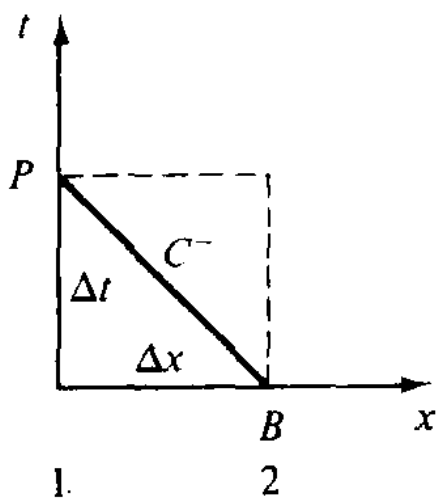

(a)

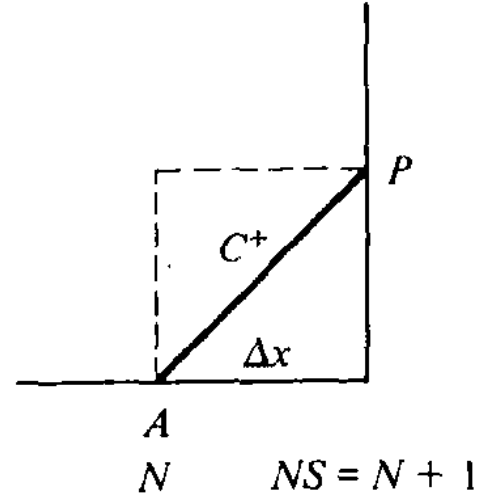

(b)

Figure 4-3: Characteristics boundaries [1]

\subsubsection{Hydraulic unit at the upstream end (pressurization of the system)}

Considering a hydraulic unit at the upstream end, for which the hydraulic grade line is assumed constant during duration of the transient flow. This boundary condition may be described as

$$
H_{1}{ }^{t+1}=H_{R}
$$

which $H_{R}$ is the elevation of hydraulic grade-line.

Since $H_{1}$ is known, $Q_{1}$ is determined by direct solution of (4-34), using the Taylor series approximation for the normal Cauchy stress tensor, (4-23).

$$
Q_{1}^{t+1}=\frac{H_{1}-C_{M}{ }^{t}}{B_{M}{ }^{t}}+\frac{c_{g}}{s_{j}}
$$

The subscript "1" refers to the upstream section, at point $P$, Figure 4-3a; $C_{M}{ }^{t}$, $B_{M}{ }^{t}, Q_{1}^{t}$ and $Q_{i+1}^{t}$ are variables in the computational procedure but are dependent only on known values from the previous time step, in this case, point $B$, section 2 [1]. 


\subsubsection{Hydraulic unit at the upstream end (depressurization of the system)}

Considering a hydraulic unit at the upstream end for which the hydraulic grade line is assumed constant during duration of the transient flow. For depressuriaztion of the system, the boundary condition may be described as

$$
H_{1}{ }^{t+1}=0
$$

Since $H_{1}$ is known, $Q_{1}$ is determined (4-42)

$$
Q_{1}^{t+1}=-\frac{C_{M}{ }^{t}}{B_{M}{ }^{t}}+\frac{c_{g}}{s_{j}}
$$

\subsubsection{Time Varying Hydraulic unit at the upstream end}

Considering a hydraulic unit at the upstream end for which the hydraulic grade line is assumed to vary with time for the duration of the transient flow. For a sinusoidal variation of $10 \sin (\pi t)$ the boundary condition may be described as

$$
\begin{aligned}
& B=\frac{c}{g A} \\
& C_{M}=H_{2}-B Q_{2}+\frac{f \Delta x}{2 g D A^{2}} Q_{2}\left|Q_{2}\right| \\
& H_{P_{1}}=H_{R}+10 \sin (\pi t) \\
& Q_{P_{1}}=\left(H_{P_{1}}-C_{M}\right) / B
\end{aligned}
$$

\subsubsection{Dead end at the downstream end}

The downstream end of a pipeline that is divided into $N$ sections is the section $N S=N+1$, Figure 4-3b. If the pipeline contains a closed end, then the boundary condition is described as

$$
Q_{N S}{ }^{t+1}=0
$$


Applying this boundary condition in (4-33) and eq. (4-24), then

$$
H_{N S}^{t+1}=C_{P}^{t}+\frac{c_{g}}{s_{j}}
$$

\subsubsection{Air Pocket at the downstream end}

Considering a pocket of air trapped in a system at the downstream end. The volume of air trapped is assumed to be small compared to the volume of liquid volume in a computing reach. The air is assumed to follow the reversible polytropic relation

$$
H_{A} \forall^{n}=C
$$

Where $H_{A}$ is the absolute head equal to the gauge pressure plus barometric pressure head,

$$
H_{A}=H_{P}-z+\bar{H}
$$

$\forall$ is the volume of trapped air, $n$ is the polytropic exponent, and $C$ is a constant. The exponent $n$ depends on the thermodynamic process followed by the gas in the vessel. If a perfect gas is assumed, at the one extreme the process may be isothermal, $n=1$, or at the other limit it may be isentropic (reversible, adiabatic), $n=1.4$. The particular situation dictates the type of process, the latter being more conservative since it predicts larger pressure changes for the same volume change. For small chambers with fast response times the process may be taken as isentropic. In larger systems with a large water volume and small air mass the transformation may approach an isothermal process. In this work an average value of 1.2 was used for simulations. Since equation (4-52) applies at any instant in time it is written at the end of a time increment by introducing the continuity equation. 


$$
\left(H_{P}+\bar{H}-z\right)\left(\forall-\Delta \mathrm{t} \frac{Q_{P}-Q}{2}\right)^{n}=C
$$

In this equation $\forall$ is the volume of air at the beginning of the time increment.

Newton's Method is used to solve equations (4-9*) and (4-53) simultaneously. When combined these two equations may be written as

$$
F_{1}=\left(C_{P}-B Q_{p}-z+\bar{H}\right)\left(\forall-Q_{P} \frac{\Delta \mathrm{t}}{2}-\frac{\mathrm{Q} \Delta \mathrm{t}}{2}\right)^{n}-C=0
$$

which is a nonlinear equation in the variable $Q_{P}$. Newton's method finds a correction to an estimated value of $Q_{P}$ by use of the expression

$$
F_{1}+\frac{d F_{1}}{D Q_{P}} \Delta Q=0
$$

where after simplification

$$
\frac{d F_{1}}{D Q_{P}}=\frac{n \Delta t C}{2\left(\forall-\frac{Q_{P}-Q}{2} \Delta \mathrm{t}\right)}-B\left(\forall-\Delta \mathrm{t} \frac{Q_{P}-Q}{2}\right)^{n}
$$

The FORTRAN code for this boundary condition may take the following form, beginning with an estimated value $Q_{P}$ of at the end of the time increment.

\subsubsection{Blockage at the downstream end}

Considering a blockage to fully impede the flow in the pipeline and to be cylinder with planar ends this condition is modeled within the MMOC as a dead end. The pressure in the last computational reach, $P_{M M O C}$, is applied to a finite element model of the blockage for the duration of a fluid time step. If the applied pressure force overcomes the static frictional forces, $F_{S}$, which keep the blockage in place movement occurs during the time step the updated position of the blockage is returned to the MMOC. This axial motion of the blockage lengthens of the last computational reach thereby increasing the 
volume of the fluid simulation. Once the plug is moving the dynamic coefficient of friction is used in place of the static.

- The friction force exerted on the blockage by the pipe walls is equal to

$\circ F_{s}=\mu_{s} N$ when stationary

○ $F_{D}=\mu_{D} N$ when in motion

- The check for the onset of motion is

$\circ\left(P_{M M O C}-P_{\text {Amb. }}\right) A_{\text {Blockage }} \leq F_{S}$

- The motion of the blockage is tracked as a particle

$\circ a_{+1 \text { Blockage }}=\left(P_{M M O C}-P_{\text {Amb. }}\right) A_{\text {Blockage }}-F_{D}$

○ $v_{\text {Blockage }}=a_{-1 \text { Blockage }} d t_{M M O C}$

○ $\Delta x_{\text {Blockage }}=v_{\text {Blockage }} d t_{M M O C}+0.5 a_{+1 \text { Blockage }} d t_{M M O C}{ }^{2}$

○ $x_{\text {Blockage }}=x_{-1 \text { Blockage }}+\Delta x_{\text {Blockage }}$

- Blockage exit criteria

○ $x_{\text {Blockage }}>x_{\text {Outlet }}$

\subsection{Pipeline Characterization through Optimization}

The process of representing a pipeline as a numerical model is herein referred to as pipeline characterization. Characterizing a pipeline requires the specification of parameters for the physical arrangement of the system such as the pipe diameter, wall thickness, modulus of elasticity, and section length. Parameters representing the environmental conditions, barometric pressure and temperature, must also be specified. All of these parameters are easily observed and measured or gathered from manufacture's 
specifications. In addition to these the parameters that describe the losses which occur as a transient propagates in the system must be specified. The principle parameter used to quantify pipeline losses is the Darcy-Weisbach friction factor [38].

The Darcy-Weisbach friction factor represents the loss of energy of a fluid traveling in a pipeline due to internal viscous dissipation and friction with the pipe walls. It is well documented for steady state flow. The surging flows in transient pipeline conditions fluctuate between the laminar, transitional, and turbulent flow regimes. In the transitional and turbulent flow the relations for the friction factor are non-linear such as the Colebrook equation. These equations are often solved by iterative methods for a particular fluid velocity and Reynolds number [17].

The proposed loss coefficient, $c_{g}$, represents the loss of energy of a fluid due to partial reflection of transient pressure pulses at small radius $90^{\circ}$ elbows. The effect of an isolated small radius $90^{\circ}$ elbow on a transient's propagation have been studied by Valentine, Phillips, and Walker [14]. The study results reveal that for low frequency waves, a partial negative pressure reflection occurs at the elbow and the strength of this reflection is of the order of $15 \%$ to $30 \%$ of that of the incoming wave; the amount of the reflection depends strongly upon the ratio of the wall thickness to the mean tube radius, is somewhat dependent upon the total bend angle, but is relatively insensitive to the radius of curvature of the elbow.

It is proposed in this thesis that the effect of multiple small radius $90^{\circ}$ elbows in a pipeline is strongly dependant on their positions in the pipeline and the lengths of straight sections of pipe which connect them. 


\subsubsection{Approach}

The values of the Darcy-Weisbach friction factor, $\mathrm{f}$, and the proposed geometric loss coefficient, $\mathrm{c}_{\mathrm{g}}$, are not known a priori for transient pipeline flows. When at least a pair of pressure sensor time histories is available for a given pipeline $f$ and $\mathrm{c}_{\mathrm{g}}$ can be determined by fitting the MMOC simulation results to the time histories. The resultant Darcy-Weisbach friction factor, $\mathrm{f}$, and geometric loss coefficient, $\mathrm{c}_{\mathrm{g}}$, along with the parameters for the physical arrangement and environmental conditions are said to characterize the pipeline.

\subsubsection{Selection of optimization method}

The objective in characterizing a pipeline is to produce a model of the pipeline that accurately predicts the transient pressure heads along the pipeline. The physical and environmental parameters defining the pipeline are fixed, the values of the DarcyWeisbach friction factor, $\mathrm{f}$, and the proposed geometric loss coefficient, $\mathrm{c}_{\mathrm{g}}$, are the design variables, and the objective is to minimize the error between the peak pressure predicted by the MMOC and that observed in the experimental pressure sensor time histories.

The Darcy-Weisbach friction factor, $\mathrm{f}$, has a non-linear relationship to pressure head across the laminar, transitional, and turbulent flow regimes. The relationship of the proposed geometric loss coefficient, $\mathrm{c}_{\mathrm{g}}$, to pressure head is not known and is likely nonlinear. Local minima are likely in such a non-linear design space. Gradient-based optimization methods may return local minima rather than the true global minima. For this reason non-gradient based methods were considered. The differential evolution algorithm presented in EML5509 "Mechanical Design Optimization" by Profs. 
Dulikravich, Colaco, and Orlande in Spring 2009 and depicted below in Figure 4-4 is a robust and efficient method [39]. Differential evolution was selected over other nongradient based methods such as simulated annealing or particle swarm for its greater efficiency in domains with numerous local minima.

\subsubsection{Implementation}

The MMOC algorithm was incorporated into the differential evolution algorithm as a design evaluation as shown below in Figure 4-4.

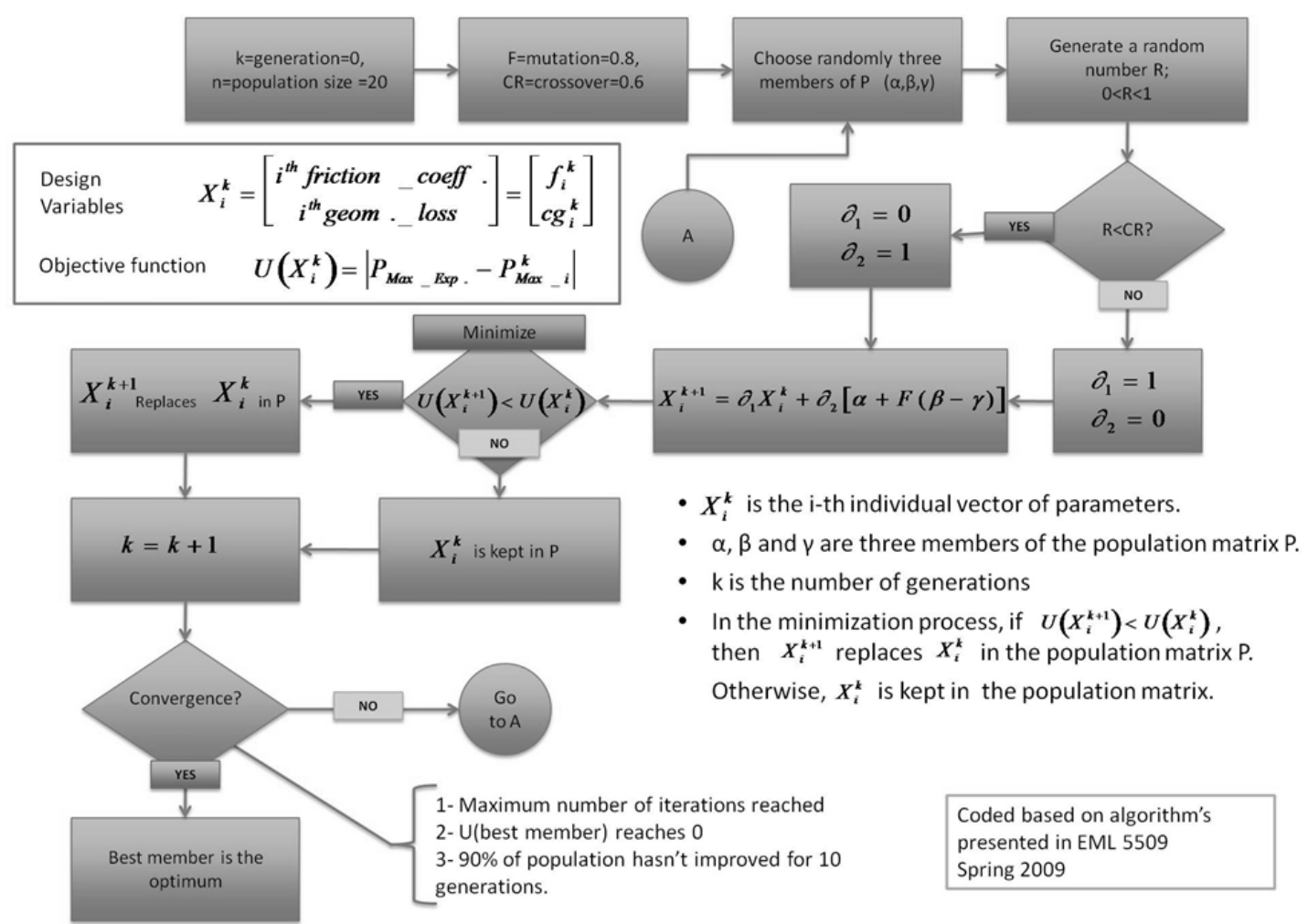

Figure 4-4: Pipeline characterization flow chart

In this implementation each design is a set of input parameters to MMOC where the majority are fixed. The only design variables are the Darcy-Weisbach friction factor, $\mathrm{f}$, and the proposed geometric loss coefficient, $\mathrm{c}_{\mathrm{g}}$. Each design is evaluated in the 
objective function for how well it predicts the maximum pressure observed in the experimental pressure sensor time histories.

Table 1: Pipeline characterization design variables

\begin{tabular}{|l|c|c|}
\hline \multicolumn{1}{|c|}{ Design Variable } & Minimum & Maximum \\
\hline Darcy-Weisbach friction factor, $\mathrm{f}$ & 0.01 & 0.05 \\
\hline Geometric loss coefficient, $\mathrm{c}_{\mathrm{g}}$ & 0.01 & 1.00 \\
\hline
\end{tabular}

As shown in Figure 4-4 optimization begins with an initial population of 20 designs of random values for Darcy-Weisbach friction factor, $f$, and geometric loss coefficient, $\mathrm{c}_{\mathrm{g}}$, within the ranges described in 
above. This first generation is evaluated in the objective function. Three members are chosen randomly to combine to form a new design in the second generation. New designs which more accurately predict the maximum pressure in the pipeline replace their predecessors in the population and the process continues until the convergence criteria are reached.

\subsection{Pump Operation Optimization}

Safe and efficient operation of a pump at the inlet to a pipeline is a clear goal in pipeline operations. Once a pipeline is characterized with MMOC as described in chapter 4.3 the model can be used to predict the transients that will be observed in the system. The objective of achieving a desired peak pressure at a specific position in the pipeline can be achieved with an appropriate pump operation schedule. The appropriate schedule for a pipeline can be determined by optimizing the pressure profile applied to the pipeline. The objective of the optimization is the minimization of the difference between the simulated peak pressure and the target peak pressure at the target location.

\subsubsection{Selection of optimization method}

The pump operation parameters, peak applied pressure and hold time, have a nonlinear relationship to the peak pressure generated in the pipeline due to the constructive and destructive wave interference between the forward and reflected pressure pulses. It is possible that over the range of the parameters shown in Table 2 that multiple local minima exist. Gradient-based optimization methods may return local minima rather than the true global minima. For this reason non-gradient based methods were considered. The differential evolution algorithm presented in EML5509 "Mechanical Design 
Optimization" by Profs. Dulikravich, Colaco, and Orlande in Spring 2009 and presented below in Figure 4-5 is a robust and efficient method. Differential evolution was selected over other non-gradient based methods such as simulated annealing or particle swarm for its greater efficiency in domains with numerous local minima.

\subsubsection{Implementation}

The MMOC algorithm was incorporated into the differential evolution algorithm as a design evaluation as shown below in Figure 4-5

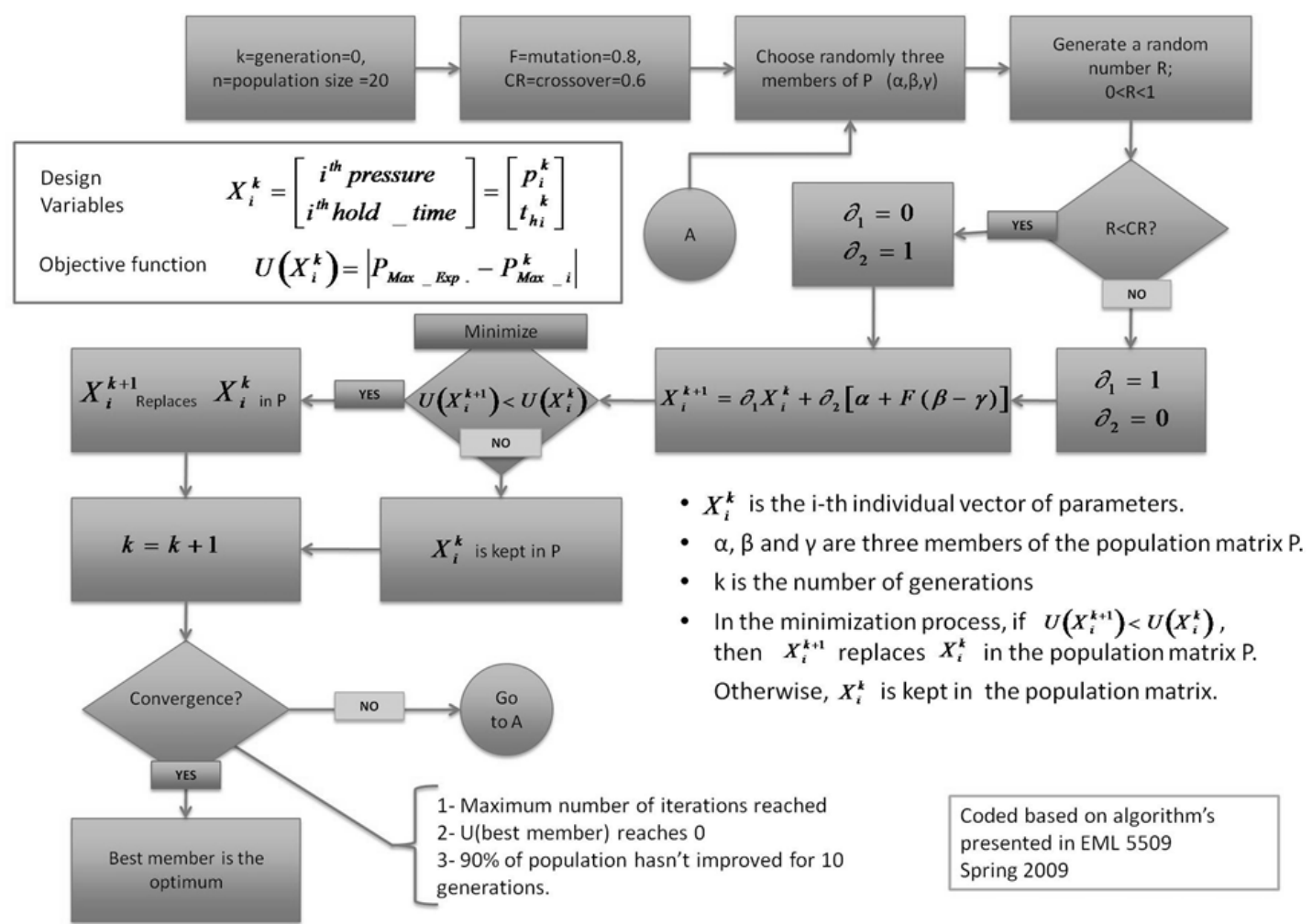

Figure 4-5: Pump Operation Optimization flow chart

In this implementation each design is a set of input parameters to MMOC where the majority are fixed. The only design variables are the peak pressure applied, $\mathrm{P}$, and the duration that the peak pressure is maintained (hold time), $\mathrm{t}_{\mathrm{h}}$. Each design is evaluated 
in the objective function for how well it predicts the maximum pressure observed in the experimental pressure sensor time histories.

Table 2: Pump Operation Design Variables

\begin{tabular}{|r|c|c|}
\hline \multicolumn{1}{|c|}{ Design Variable } & Minimum & Maximum \\
\hline 285ft and 621ft Test Cases & & 60 \\
\hline Peak pressure applied, P (Psi) & 20 & 10 \\
\hline Hold time, $\mathrm{t}_{\mathrm{h}}(\mathrm{s})$ & 0.1 & \\
\hline $1797 \mathrm{ft}$ Test Case & & 60 \\
\hline Peak pressure, $\mathrm{P}(\mathrm{Psi})$ & 20 & 15 \\
\hline Hold time, $\mathrm{t}_{\mathrm{h}}(\mathrm{s})$ & 5 & \\
\hline
\end{tabular}

As shown in Figure 4-5 optimization begins with an initial population of 20 designs of random values for peak pressure applied, $\mathrm{P}$, and hold time, $\mathrm{t}_{\mathrm{h}}$, within the ranges described in Table 2above. This first generation is evaluated in the objective function. Three members are chosen randomly to combine to form a new design in the second generation. New designs which more accurately predict the maximum pressure in the pipeline replace their predecessors in the population and the process continues until the convergence criteria are reached.

\subsection{Blockage Detection}

\subsubsection{Assumptions}

- The geometry and anchoring conditions of the pipeline is known. 
- Pipeline is fully blocked by a single cylindrically shaped elastic material with an outer diameter equal to the inner diameter of the pipeline but is otherwise clean and free of partial blockages.

- The blockage is stationary and perfectly reflects the transient pulse.

- Access to the pipeline for instrumentation is limited to the inlet of the pipeline.

- The coefficients of friction and geometric loss have been determined for the pipeline when it was unblocked.

\subsubsection{Approach}

The experimental data from the pressure sensor at the inlet is compared with the pressure predicted in the MMOC model of a pipeline with at blockage at the outlet of the pipeline. If the peak pressure at the inlet in the simulated data does not match the experimental data the last segment of the pipeline is shortened by $10 \%$ of its length and the MMOC is simulation is re-run. This progressive shortening of the last pipeline segment continues until the peak pressure at the inlet in the simulation matches the peak pressure at the inlet in the experimental data and the blockage is located or the segment is completely removed. If the blockage was not located in the last segment then the pipeline continues to be shortened in increments of $10 \%$ of the last remaining segment until the blockage is located.

This approach enables the blockage to be located with limited access to the pipeline while considering the varying wave speeds in the pipeline due to varying pipe diameters, materials, anchoring, and or elbows. 


\subsubsection{Implementation}

Considering a limited access pipeline typical of DOE transfer lines the blockage can be located by recording the pressure at the pump and at the inlet to the pipeline. For fluid and pipeline combinations with wave speeds on the order of $1400 \mathrm{~m} / \mathrm{s}$ the pump

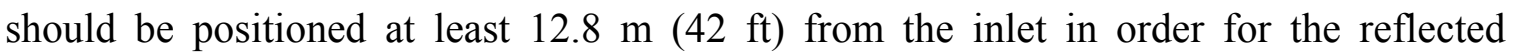
transient to be clearly distinguished from the forward transient.

A short duration transient pressure pulse is initiated at the pump, propagates through the pipeline and is reflected by the blockage. The sensors at the pump and the inlet record the time history of the pressure at each location. The data is input into the MMOC model. The experimental data from the pressure sensor at the inlet is compared with the pressure predicted in the MMOC model of a pipeline with at blockage at the outlet of the pipeline. If the peak pressure at the inlet in the simulated data does not match the experimental data the last segment of the pipeline is shortened by $10 \%$ of its length and the MMOC is simulation is re-run. This progressive shortening of the last pipeline segment continues until the peak pressure at the inlet in the simulation matches the peak pressure at the inlet in the experimental data and the blockage is located or the segment is completely removed. If the blockage was not located in the last segment then the pipeline continues to be shortened in increments of $10 \%$ of the last remaining segment until the blockage is located.

\subsection{Blockage Removal}

\subsubsection{Assumptions}

- The pipeline is rigid. 
- The pipeline is fully blocked by a cylindrical shaped elastic material with an outer diameter equal to the inner diameter of the pipeline and length of 1 meter.

- The pipeline is evacuated of fluid downstream of the blockage where ambient pressure exists.

- The pressure differential across the blockage acts to compress and dilate it.

- The upstream and downstream ends of the blockage remain planar.

- The circumferential surface of the blockage remains that of a cylinder.

- The dilation of the blockage increases the normal force the blockage exerts on the pipe in which it is lodged.

- The friction force exerted on the blockage by the pipe walls is equal to

$\circ F_{S}=\mu_{S} N$ when stationary

○ $F_{D}=\mu_{D} N$ when in motion

- The blockage moves as a single cylindrical volume.

\subsubsection{Approach}

The blockage is considered to be a collection of infinitely many particles interacting together through contact as a deformable body inside of a rigid pipe. The blockage has distributed forces acting on its boundaries; hydraulic pressure at the upstream end, ambient pressure at the downstream end and friction on the circumference. The pressure forces act normal to the upstream and downstream faces. The friction forces act to resist the motion of the blockage. 
The standard development of the equilibrium equation for a general three dimensional body can be found in the excellent introductory text by Krysl [40]. The result is

$$
\int_{V} \rho \frac{d \vec{v}}{d t} d V=\int_{V} \vec{b} d V+\int_{V} \beta^{T} \vec{\sigma} d V \Rightarrow \rho \frac{d \vec{v}}{d t}=\vec{b}+\beta^{T} \vec{\sigma}
$$

where $m$, is the mass, $V$, is the volume and $S$, is the bounding surface of the volume $V . \vec{b}$ is body force and $\vec{t}$ are the distributed forces or tractions acting on the bounding surface $S$. The six independent components of the Cauchy stress tensor. These six components are stated conveniently as a stress vector [40] in Cartesian coordinates for simplicity

$$
\vec{\sigma}=\left[\sigma_{x}, \sigma_{y}, \sigma_{z}, \tau_{x y}, \tau_{x z}, \tau_{y z}\right]^{T}
$$

$\beta^{T}$ is the stress divergence operator [40]

$$
\beta_{3 D}^{T}=\left[\begin{array}{cccccc}
\partial / \partial x & 0 & 0 & \partial / \partial y & \partial / \partial z & 0 \\
0 & \partial / \partial y & 0 & \partial / \partial x & 0 & \partial / \partial z \\
0 & 0 & \partial / \partial z & 0 & \partial / \partial x & \partial / \partial y
\end{array}\right]
$$

This statement of the dynamic equilibrium of a point particle (4-57) [40] represents the inertial force (mass times acceleration) on the left and the body force and the force generated by a stress gradient across the particle and the right. The stress term will be replaced by the measurable strains through the use of the symmetric gradient or strain displacement operator, $\beta$, which is the un-transposed stress divergence operator $\beta^{T}$.

$$
\epsilon=\beta u
$$


where $u$ is the displacement vector. The strain displacement operator, $\beta$, links displacements in terms of their components in the global Cartesian basis to strains.

The blockage material is modeled at isotropic in this work and as such has a material stiffness matrix in $3 \mathrm{D}, D_{3 D}[40]$

$$
D_{3 D}=\left[\begin{array}{cccccc}
\lambda+2 G & \lambda & \lambda & 0 & 0 & 0 \\
\lambda & \lambda+2 G & \lambda & 0 & 0 & 0 \\
\lambda & \lambda & \lambda+2 G & 0 & 0 & 0 \\
0 & 0 & 0 & G & 0 & 0 \\
0 & 0 & 0 & 0 & G & 0 \\
0 & 0 & 0 & 0 & 0 & G
\end{array}\right]
$$

Where the shear modulus, $G=\frac{E}{2(1+v)}$ and the Lamé constant is $\lambda=\frac{E v}{(1+v)(1-2 v)}$

The derivation of the dynamic equilibrium of a point particle in terms of measurable strains can be simplified for the blockage from its statement in three dimensions to two dimensions due to its axial symmetry. First the equilibrium equations are stated in cylindrical coordinates by letting $\mathrm{r}=$ radial direction, $\mathrm{z}=$ axial direction and $\theta=$ circumferential direction. In terms of shear stresses, $\tau$, normal stress, $\sigma$, body force, $\vec{b}$, mass density, $\rho$, and acceleration, $\ddot{u}$

$$
\begin{aligned}
& \frac{1}{r} \frac{\partial \sigma_{\theta}}{\partial \theta}+\frac{\partial \tau_{r \theta}}{\partial r}+\frac{2 \tau_{r \theta}}{r}+\frac{\partial \tau_{z \theta}}{\partial z}+\vec{b}_{\theta}=\rho \ddot{u}_{\theta} \\
& \frac{\partial \sigma_{r}}{\partial r}+\frac{1}{r} \frac{\partial \tau_{r \theta}}{\partial \theta}+\frac{\sigma_{r}-\sigma_{\theta}}{r}+\frac{\partial \tau_{z \theta}}{\partial z}+\vec{b}_{r}=\rho \ddot{u}_{r} \\
& \frac{\partial \sigma_{z}}{\partial z}+\frac{1}{r} \frac{\partial \tau_{z \theta}}{\partial \theta}+\frac{\partial \tau_{r z}}{\partial r}+\frac{\tau_{r z}}{r}+\vec{b}_{z}=\rho \ddot{u}_{z}
\end{aligned}
$$

The constitutive equations that relate stress to strain are 


$$
\begin{aligned}
& \sigma_{r}=\frac{E}{(v+1)(2 v-1)}\left[(v-1) \varepsilon_{r}-v\left(\varepsilon_{\theta}+\varepsilon_{z}\right)\right] \\
& \sigma_{\theta}=\frac{E}{(v+1)(2 v-1)}\left[(v-1) \varepsilon_{\theta}-v\left(\varepsilon_{r}+\varepsilon_{z}\right)\right] \\
& \sigma_{z}=\frac{E}{(v+1)(2 v-1)}\left[(v-1) \varepsilon_{z}-v\left(\varepsilon_{r}+\varepsilon_{\theta}\right)\right]
\end{aligned}
$$

Krysl provides a clear development of the standard simplifications for axisymmetric volumes [40]. The result of the simplification is (4-68) and (4-69)

$$
\begin{aligned}
& \frac{\partial \sigma_{r}}{\partial r}+\frac{\partial \tau_{r z}}{\partial r}+\frac{\sigma_{\theta}}{r}+\vec{b}_{r}=\rho \ddot{u}_{r} \\
& \frac{\partial \tau_{r z}}{\partial r}+\frac{\partial \sigma_{z}}{\partial z}+\vec{b}_{z}=\rho \ddot{u}_{z}
\end{aligned}
$$

Which can be stated in matrix form [40]

$$
\rho\left\{\begin{array}{l}
\ddot{u}_{r} \\
\ddot{u}_{z}
\end{array}\right\}=\left[\begin{array}{cccc}
\partial / \partial r & 0 & 1 / r & \partial / \partial z \\
0 & \partial / \partial z & 0 & \partial / \partial r
\end{array}\right]\left\{\begin{array}{c}
\sigma_{r} \\
\sigma_{z} \\
\sigma_{\theta} \\
\tau_{r z}
\end{array}\right\}+\left\{\begin{array}{l}
\vec{b}_{r} \\
\vec{b}_{z}
\end{array}\right\}
$$

For analysis of the blockage in this work gravity and other body forces are neglected, $\left\{\begin{array}{l}\vec{b}_{r} \\ \vec{b}_{z}\end{array}\right\}=\left\{\begin{array}{l}0 \\ 0\end{array}\right\}$. The larger time step required in the MMOC algorithm for the fluid in the pipe enables the analysis of the blockage to be static and accelerations neglected, $\left\{\begin{array}{l}\ddot{u}_{r} \\ \ddot{u}_{z}\end{array}\right\}=\left\{\begin{array}{l}0 \\ 0\end{array}\right\}$. The constitutive equation for an axisymmetric volume may then be stated as [40] 


$$
\left\{\begin{array}{c}
\sigma_{r} \\
\sigma_{z} \\
\sigma_{\theta} \\
\tau_{r z}
\end{array}\right\}=\left[\begin{array}{cccc}
\lambda+2 G & \lambda & \lambda & 0 \\
\lambda & \lambda+2 G & \lambda & 0 \\
\lambda & \lambda & \lambda+2 G & 0 \\
0 & 0 & 0 & G
\end{array}\right]\left\{\begin{array}{c}
\epsilon_{r} \\
\epsilon_{z} \\
\epsilon_{\theta} \\
\gamma_{r z}
\end{array}\right\}
$$

To solve equations (4-70) and (4-71) the variational principles in the small displacement theory of elasticity [41] will be used by letting

$\mathrm{r}, \mathrm{u}=$ radial direction, radial displacement

$\mathrm{z}, \mathrm{v}=$ axial direction, axial displacement

$\theta, \mathrm{w}=$ circumferential direction, circumferential displacement

The principle of virtual work for axisymmetric volumes may be stated as [41]

$\delta \iiint_{V} A_{A x i}(u, v) d V-\iiint_{V}\left(\vec{b}_{r} \delta u+\vec{b}_{z} \delta v\right) d V$

$$
-\iint_{S}(\bar{r} \delta u+\bar{z} \delta v) d S=0
$$

Where $\iiint_{V}\left(\vec{b}_{r} \delta u+\vec{b}_{z} \delta v\right) d V=0$ since body forces are neglected and

$$
\begin{array}{r}
A_{A x i}(u, v)=\frac{E v}{2(1+v)(1-2 v)}\left(\frac{\partial u}{\partial r}+\frac{\partial v}{\partial z}\right)^{2}+G\left[\left(\frac{\partial u}{\partial r}\right)^{2}+\left(\frac{\partial v}{\partial z}\right)^{2}\right] \\
+\frac{G}{2}\left(\frac{\partial u}{\partial z}+\frac{\partial v}{\partial r}\right)^{2}
\end{array}
$$

\subsubsection{Implementation}

The statement of the principle of virtual work for volumes [41] is implemented in MeshFree [42] through Microsoft Visual C++ 2010 Express. meshFree is a mesh-less 
method that exactly treats all given boundary conditions by utilizing the Rvachev's Function Method (RFM). The RFM utilizes approximate distance functions that vanish on the corresponding geometric boundaries of the model to exactly satisfy the boundary conditions. The approximate distance functions, boundary conditions, and basis functions are assembled into a solution structure. The solution structure contains the necessary degrees of freedom in the form of coefficients of the basis functions. Linear combinations of basis functions are used to approximate the differential equations in the problem. Any sufficiently complete system of basis functions can be used: polynomials. B-splines, functions forming a partition of unity, or even finite element functions [5]. In this work 2D B-splines are used.

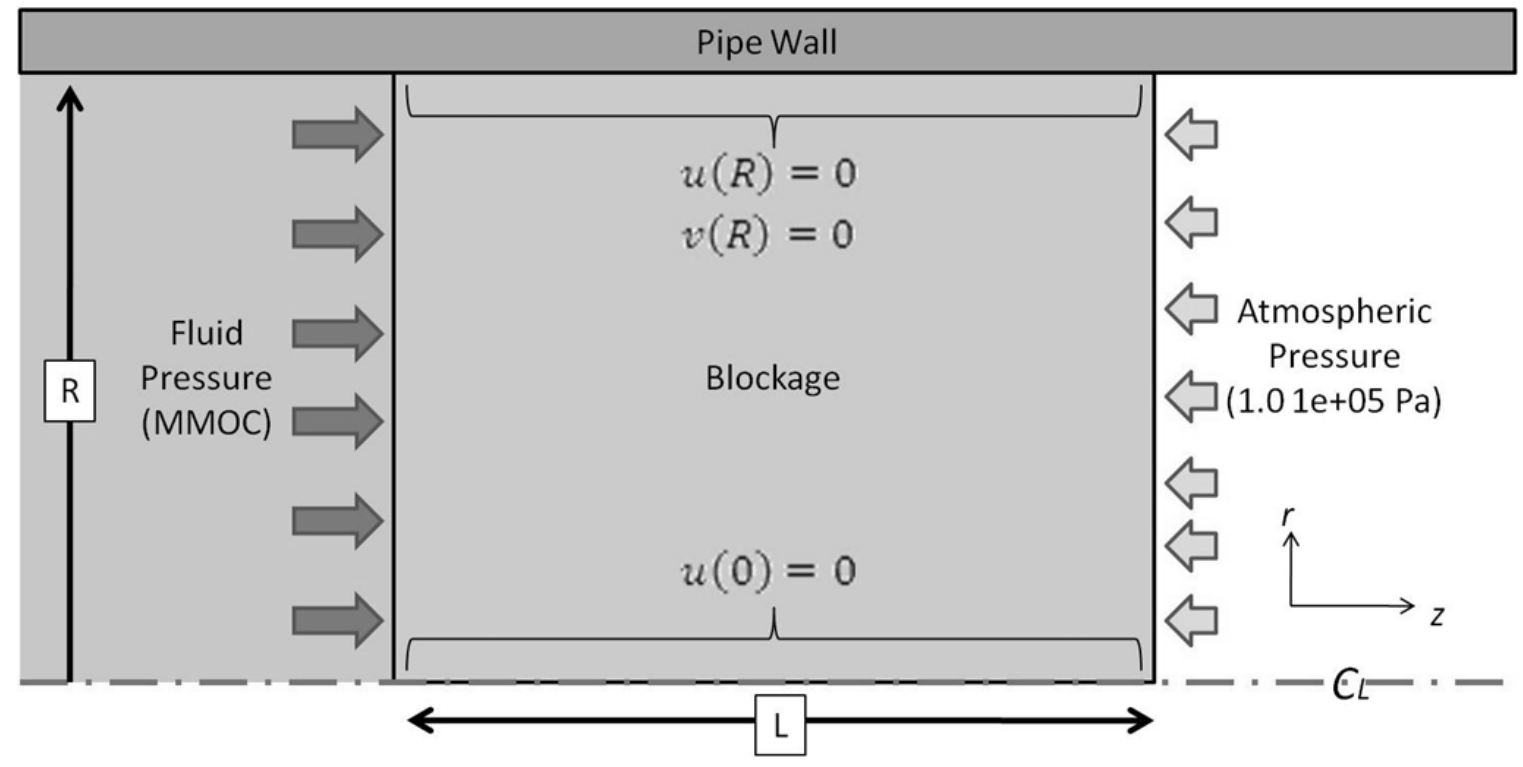

Figure 4-6: Boundary Conditions for Blockage Removal

The blockage is modeled in meshFree as an axisymmetric slice of a cylinder as shown in Figure 4-6. The blockage is constrained from radial expansion by the rigid pipe walls and is loaded by the fluid pressure on one end and the atmospheric pressure on the 
other. The boundary conditions in the radial direction are modeled as a line of symmetry along the axis of the pipe and blockage and as a Dirichlet condition at the pipe wall. The boundary conditions in the axial direction at the blockage faces are represented as natural conditions. Table 3 shows the mathematical formulation and the corresponding solution structure for the Dirichlet conditions used in this work. Where $w=0$ on the boundary and is positive elsewhere. is a linear combination of basis functions with unknown coefficients .

\section{Table 3: meshFree Solution Structures}

\begin{tabular}{|l|l|l|}
\hline Boundary Condition & Mathematical Formulation & $\begin{array}{l}\text { Corresponding Solution } \\
\text { Structure }\end{array}$ \\
\hline General Dirichlet & & \\
\hline Wall & & \\
\hline Symmetry & & \\
\hline
\end{tabular}

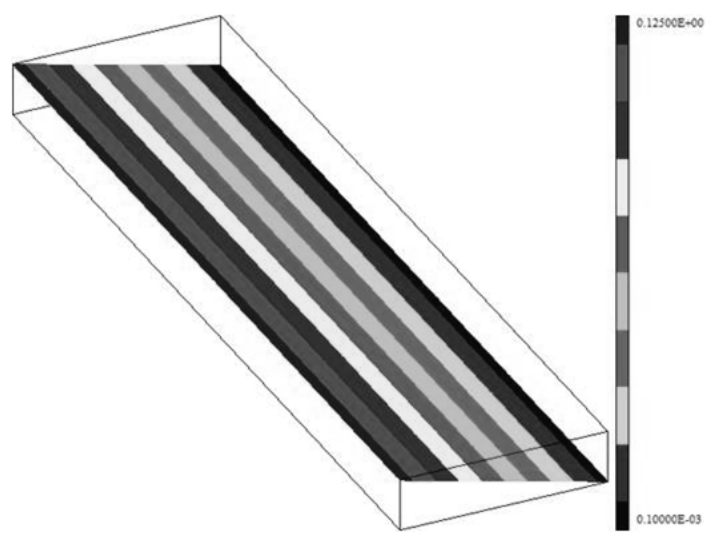

Figure 4-7: R-Function for Symmetry Boundary

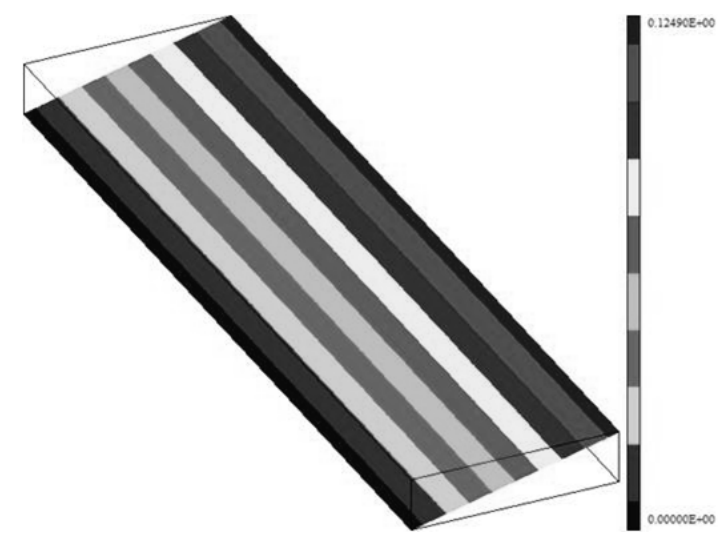

Figure 4-8: R-function for Wall Boundary 


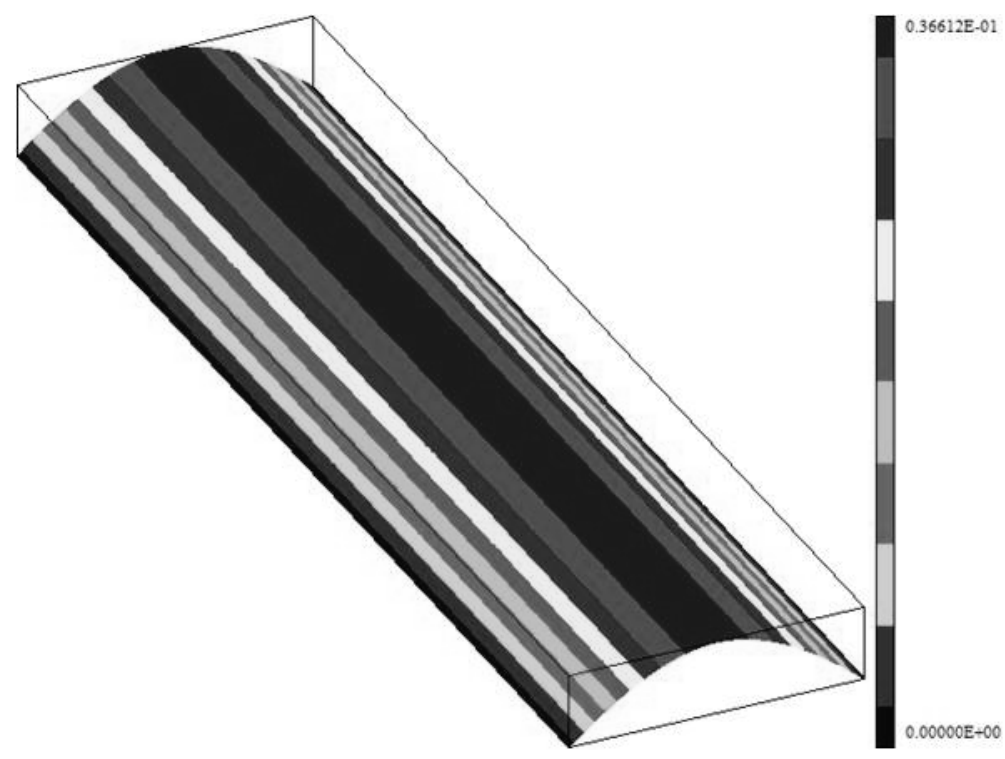

Figure 4-9: Combined R-Function for Blockage in the Radial Direction

Figure 4-7 and Figure 4-8 show the Rvachev's functions for the symmetry boundary and the wall boundary respectively. Figure 4-9 shows the intersection of the wall and symmetry boundary condition Rvachev's functions. This combined Rvachev's function is used to form the solution structure in the radial direction as

In the axial direction the blockage is free to deform along the symmetry plane but is the fixed along the pipe wall. The solution structure for the axial direction is

The stress distribution on the surface of the blockage adjacent to the pipe wall is integrated to determine the normal force applied to the pipe wall. The normal force is returned to the MMOC Blockage boundary condition algorithm as described in section 4.2.1.6. 


\subsection{Web Application Development}

\subsubsection{Resources}

The MAIDROC Laboratory hosts a high performance computing cluster, Tesla. This computing resource has $250+$ CPU's, 300+ GB RAM and 8.5+ TB of distributed and shared hard drive space. The cluster is connected to the FIU internet and the world wide web through a web portal machine, MAIDROC, which hosts the MAIDROC Laboratory website [43]. The website includes cluster monitoring pages which utilize PHP: Hypertext Preprocessor (PHP) on an Apache web server. PHP is a general-purpose scripting language originally designed for web development to produce dynamic web pages. For this purpose, PHP code is embedded into the HTML source document and interpreted by a web server with a PHP processor module, which generates the web page document. It also has evolved to include a command-line interface capability and can be used in standalone graphical applications.

\subsubsection{Approach}

The input files needed by MMOC require the specification of several parameters:

- 5 for each pipe, length, diameter, friction factor, orientation and inclination

- 2 to describe the initial condition of the flow in the pipeline, flow rate and pressure datum

- 2 selections for the boundary conditions that then may require additional parameters

- 3 for the environmental conditions, ambient pressure and temperature and gravitational acceleration 
- 3 for the solution procedure, time step, the maximum reach adjustment, and the termination time

For long pipelines the input process can be tedious and error prone. A PHP input enables each field to be checked for values within allowable ranges, for illegal characters and for being left blank. This error checking routine prompts the user to correct erroneous entries and or fill in blank fields and save valuable time by preventing simulations with poor input data from being run. The feed back to the user also expedites the trouble shooting process.

Post processing the results of a MMOC simulation requires generating plots of the pressure and flow rate time histories from the respective output files. These files are often large, several megabytes of tab-delimited floating point numbers, for long pipelines or lengthy simulations. It is common that the files are so large that Microsoft Excel lags considerably when preparing plots for them if it can at all. GNUplot [44], a freely available open-source plotting utility is capable of handling large data sets, is highly customizable, and can be utilized interactively or through script files. The ability to generate plots in an automated routine with GNUplot is ideal for rapid visualization of simulation results from MMOC.

\subsubsection{Implementation}

A password protected web site was developed to enable authorized users of the Tesla cluster in the MAIDROC Laboratory to create, load, and edit simulations, launch them on the cluster, and visualize their results in minutes. The web site is a proof of 
concept that engineering simulation software can be driven by users through a web interface. 


\section{CHAPTER V}

\section{RESULTS AND DISCUSSION}

\subsection{Pipeline Characterization}

During the technology evaluation of NuVision's Fluidic Wave Action Technology at FIU's ARC the $285 \mathrm{ft}$ test pipeline was constructed and utilized as shown in Figure 5-1. Measurements from Pressure transducers P1 to P13 positioned as shown in Figure 5-1 were recorded during the evaluation and used as the input to MMOC. A Piecewise least squares fit to the data from P1 is used as the inlet boundary condition in MOC and MMOC.

\subsubsection{5ft Test Pipeline}

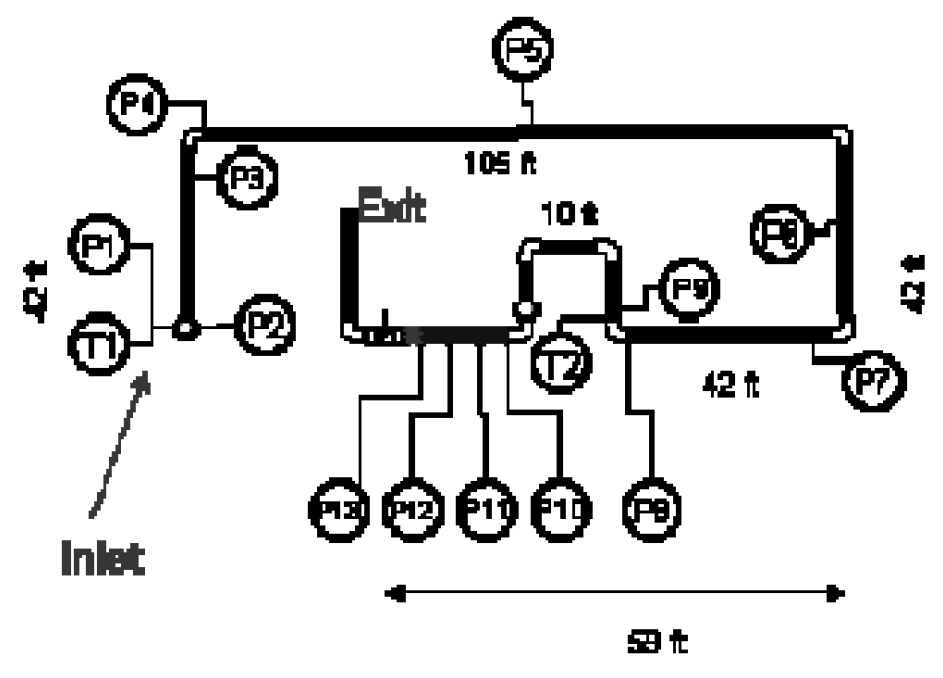

Figure 5-1: Schematic of 285ft Pipeline [3]

The MMOC model of the $285 \mathrm{ft}$ pipeline is shown in Figure 5-2. The experimental data from one trail in the technology evaluation is shown in Figure 5-3 and 
Figure 5-4. The best results attainable through optimization of the unmodified MOC, are shown in Figure 5-3 and documented in Table 4 alongside the results from MMOC.

Pipeline for MuVision 285ft Case

Nuvision $285 f t \cdots \cdots$

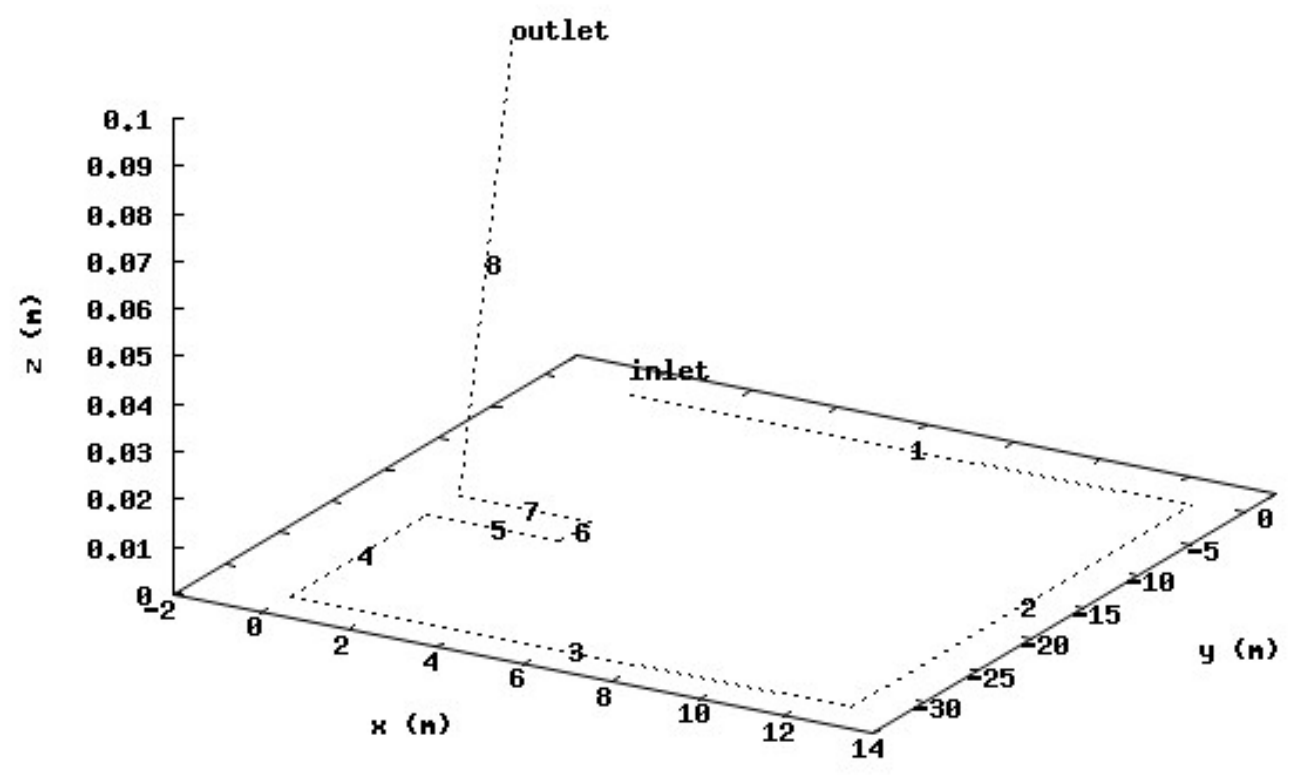

Figure 5-2: Pipeline Model for $285 \mathrm{ft}$ Case

Table 4 shows the increase in accuracy of an order of magnitude by including the proposed geometric loss coefficient to account for the effect of $90^{\circ}$ elbows in the pipeline. Figure 5-3 and Figure 5-4 show that in addition to more accurately predicting the peak pressure the addition of the geometric loss coefficient more accurately captures the transient wave form and the total energy propagating through the pipeline. The final results were obtained after 20 generations of optimization. 
Table 4: Comparison of MOC and MMOC for $285 \mathrm{ft}$ Case

\begin{tabular}{|c|c|c|}
\hline & Pressure Error (Psi) & Time Error (s) \\
\hline Method of Characteristics & & \\
\hline Friction Coefficient, $f=0.021$ & 8.6 & 0.8 \\
\hline Modified Method of Characteristics & & 0.03 \\
\hline Friction Coefficient, $f=0.0232$ & \multirow{2}{*}{0.2} & \\
\hline Geometric Loss Coeff., $c_{g}=0.282$ & & \\
\hline
\end{tabular}

Figure 5-5 displays the difference in pressure between the MMOC simulation and the experimental data. Figure 5-6 displays the difference in time of occurrence of the peak pressure between the MMOC simulation and the experimental data. In both figures the results are shown for the range of friction coefficients and geometric loss coefficients which were available to the genetic algorithm during optimization. Examination of Figure 5-5 reveals the family of values for friction coefficients and geometric loss coefficients which produce accurate pressure predictions from the MMOC simulation. Examination of Figure 5-6 reveals the family of values for friction coefficients and geometric loss coefficients which produce accurate time of peak pressure predictions from the MMOC simulation. It is notable that the families of values for accurate pressure and time predictions overlap substantially for this test pipeline. 

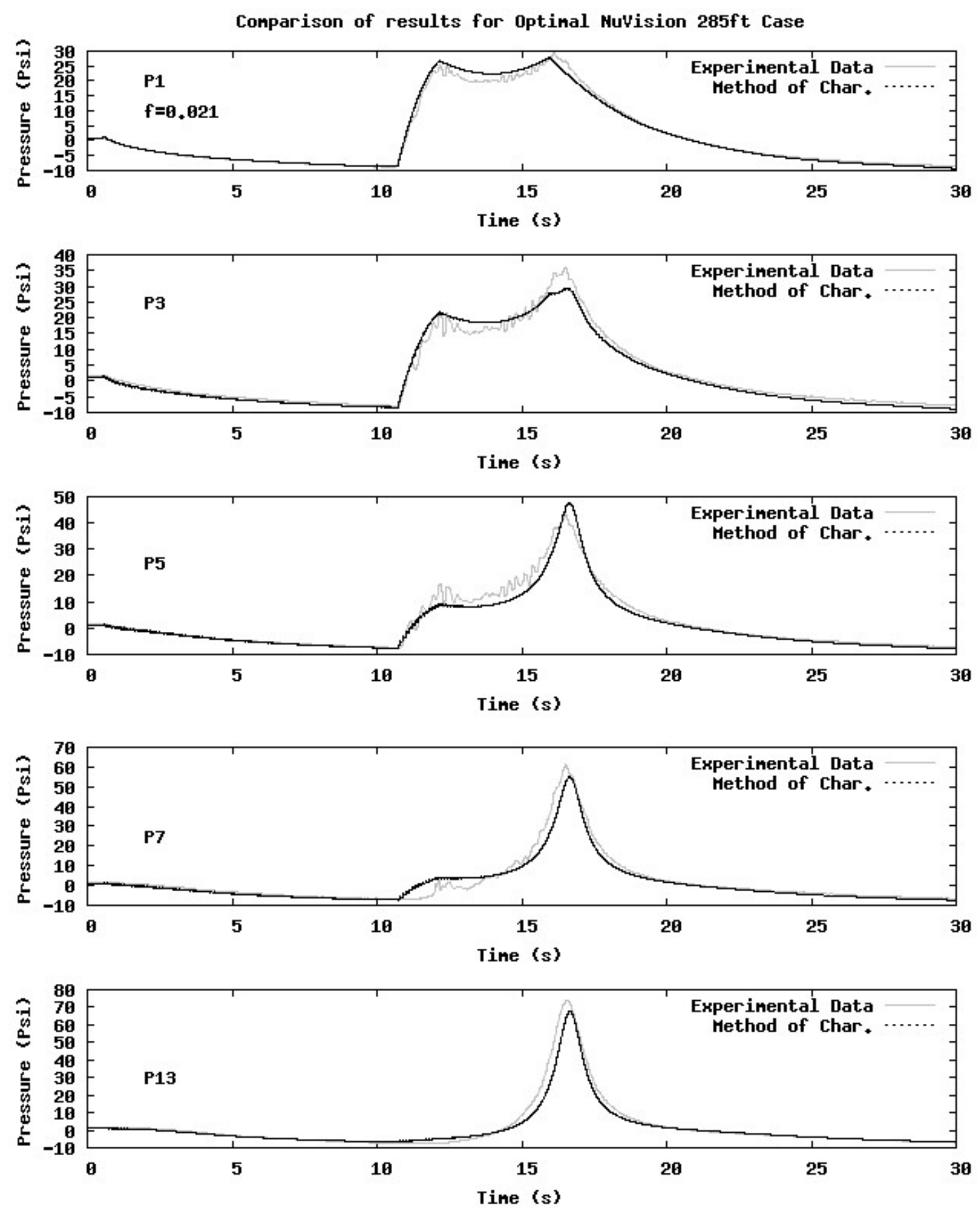

Figure 5-3: Comparision of MOC and Experimental Results for NuVision 285ft Pipeline 

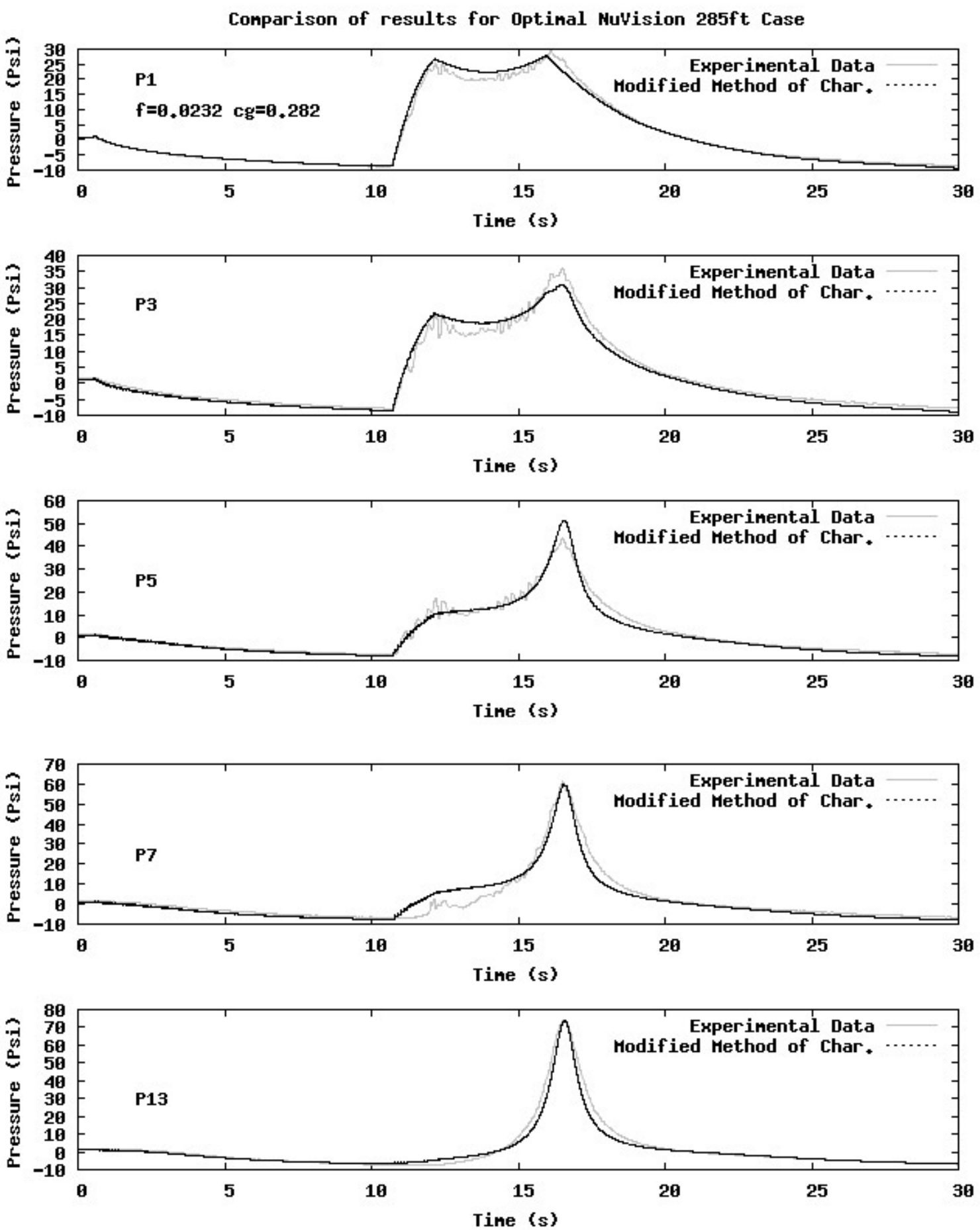

Figure 5-4:Comparision of MMOC and Experimental Results for NuVision $285 \mathrm{ft}$ Pipeline 


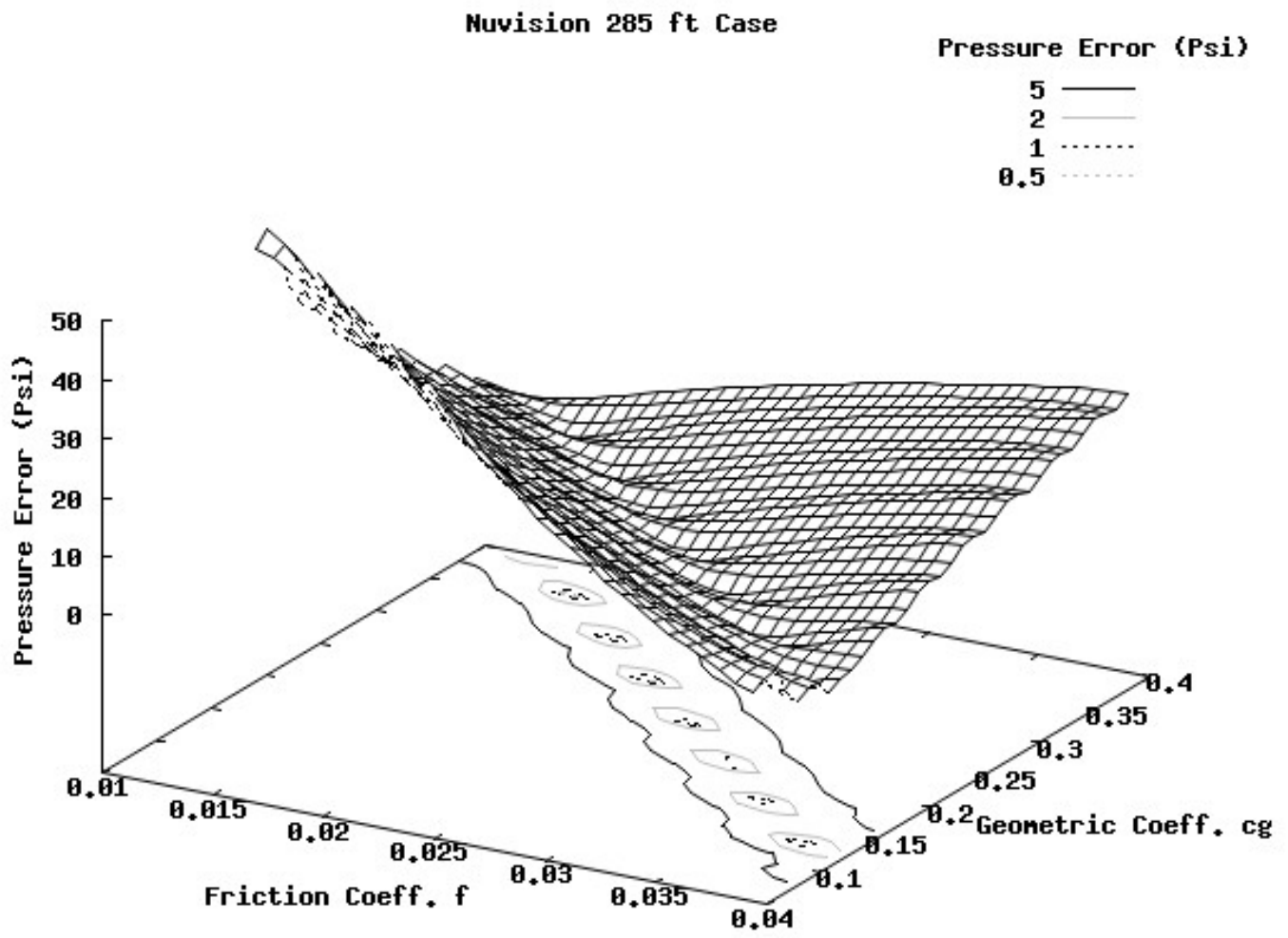

Figure 5-5: Characterization of NuVision 285ft Pipeline by Pressure 

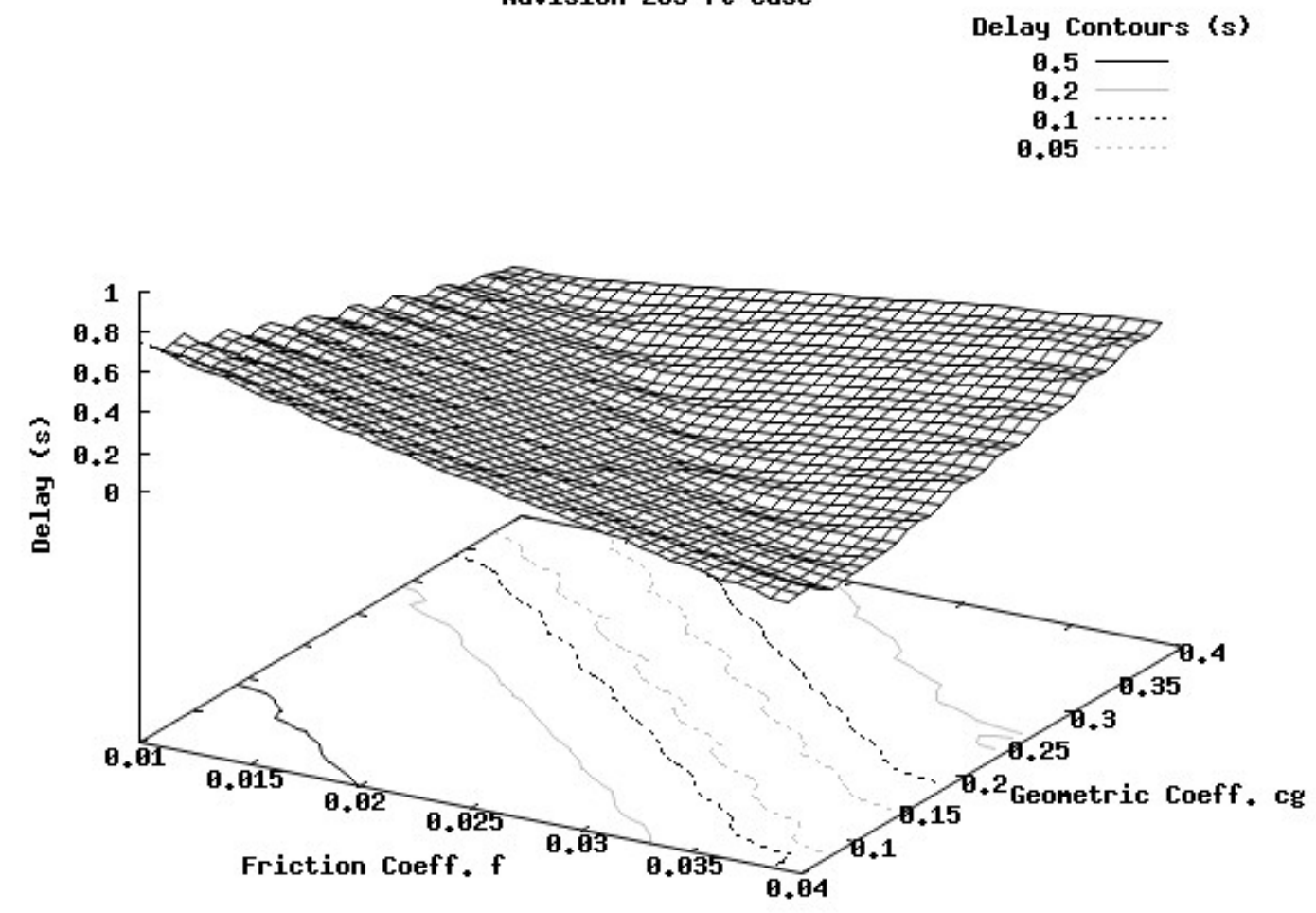

Figure 5-6: Characterization of NuVision 285ft Pipeline by Time Error

\subsubsection{1ft Test Pipeline}

During the technology evaluation of NuVision's Fluidic Wave Action Technology at FIU's ARC the $621 \mathrm{ft}$ test pipeline was constructed and utilized as shown in Figure 5-7. Measurements from Pressure transducers P1 to P13 positioned as shown in Figure 5-7 were recorded during the evaluation and used as the input to MMOC. A Piecewise least squares fit to the data from P1 is used as the inlet boundary condition in MOC and MMOC. 


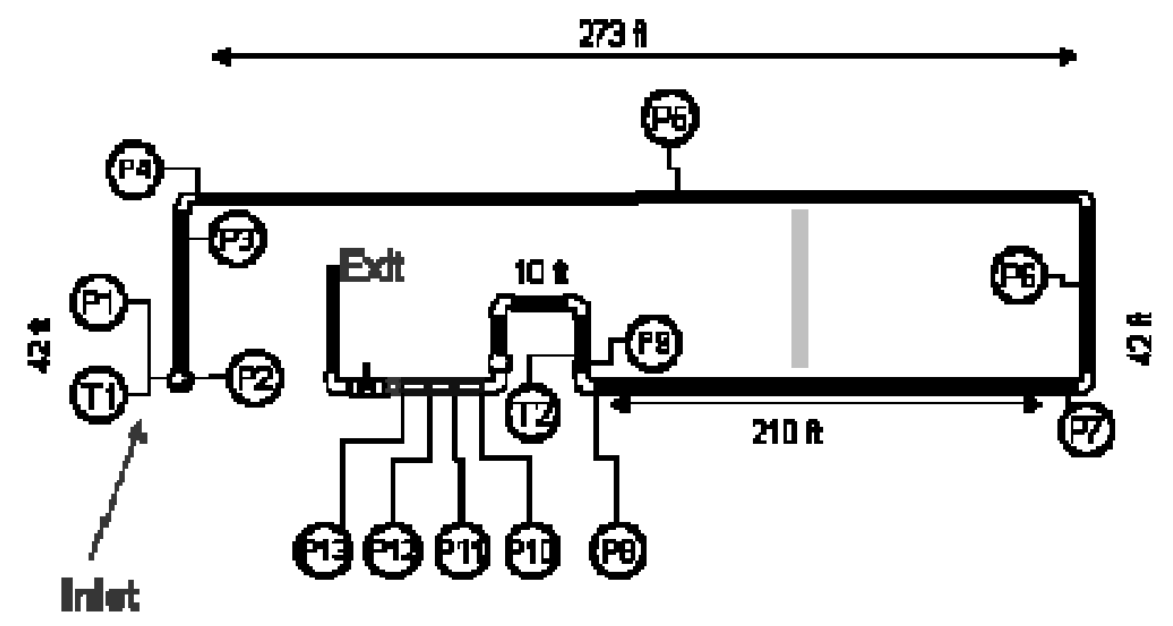

Figure 5-7: Schematic of 621ft Pipeline [3]

The MMOC model of the $621 \mathrm{ft}$ pipeline is shown in Figure 5-8. This case differs from the $285 \mathrm{ft}$ test configuration in two significant ways. Firstly, pipes 2 and 4 are significantly longer than those in the $285 \mathrm{ft}$ test case. Secondly, the pump operation schedule has a greater peak pressure and a duration that is $150 \%$ of the $285 \mathrm{ft}$ case. The experimental data from one trail in the technology evaluation is shown in Figure 5-9 and Figure 5-10. The best results attainable through optimization of the unmodified MOC, are shown in Figure 5-9 and documented in 
Table 5 alongside the results from MMOC.

Pipeline for MuVision 621ft Case

Nuvision 621ft $\cdots \cdots$

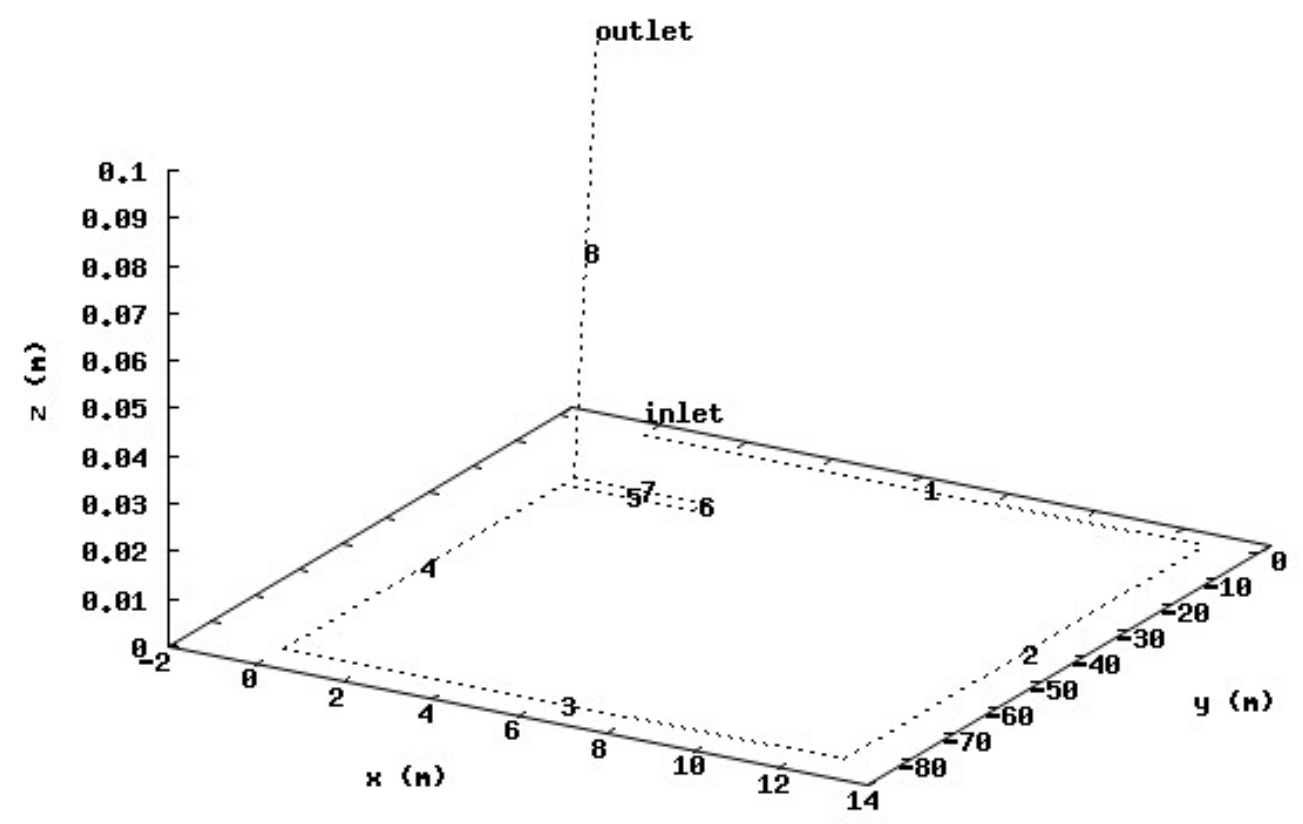

Figure 5-8: Pipeline Model for $621 \mathrm{ft}$ Case 
Table 5 shows the increase in accuracy of an order of magnitude by including the proposed geometric loss coefficient to account for the effect of $90^{\circ}$ elbows in the pipeline. Figure 5-9 and Figure 5-10 show that in addition to more accurately predicting the peak pressure the addition of the geometric loss coefficient more accurately captures the transient wave form and the total energy propagating through the pipeline. 
Table 5: Comparison of MOC and MMOC for $621 \mathrm{ft}$ Case

\begin{tabular}{|c|c|c|}
\hline & Pressure Error (Psi) & Time Error (s) \\
\hline Method of Characteristics & & \\
\hline Friction Coefficient, $f=0.021$ & 10.4 & 1.2 \\
\hline Modified Method of Characteristics & & \\
\hline Friction Coefficient, $f=0.0334$ & \multirow{2}{*}{0.6} & 0.12 \\
\hline Geometric Loss Coeff., $c_{g}=0.15$ & & \\
\hline
\end{tabular}

Figure 5-11 displays the difference in pressure between the MMOC simulation and the experimental data. Figure 5-12 displays the difference in time of occurrence of the peak pressure between the MMOC simulation and the experimental data. In both figures the results are shown for the range of friction coefficients and geometric loss coefficients which were available to the genetic algorithm during optimization. Examination of Figure 5-11 reveals the family of values for friction coefficients and geometric loss coefficients which produce accurate pressure predictions from the MMOC simulation. Examination of Figure 5-12 reveals the family of values for friction coefficients and geometric loss coefficients which produce accurate time of peak pressure predictions from the MMOC simulation. It is notable that the families of values for accurate pressure and time predictions overlap less substantially for this test pipeline than they do for the $285 \mathrm{ft}$ pipeline. 

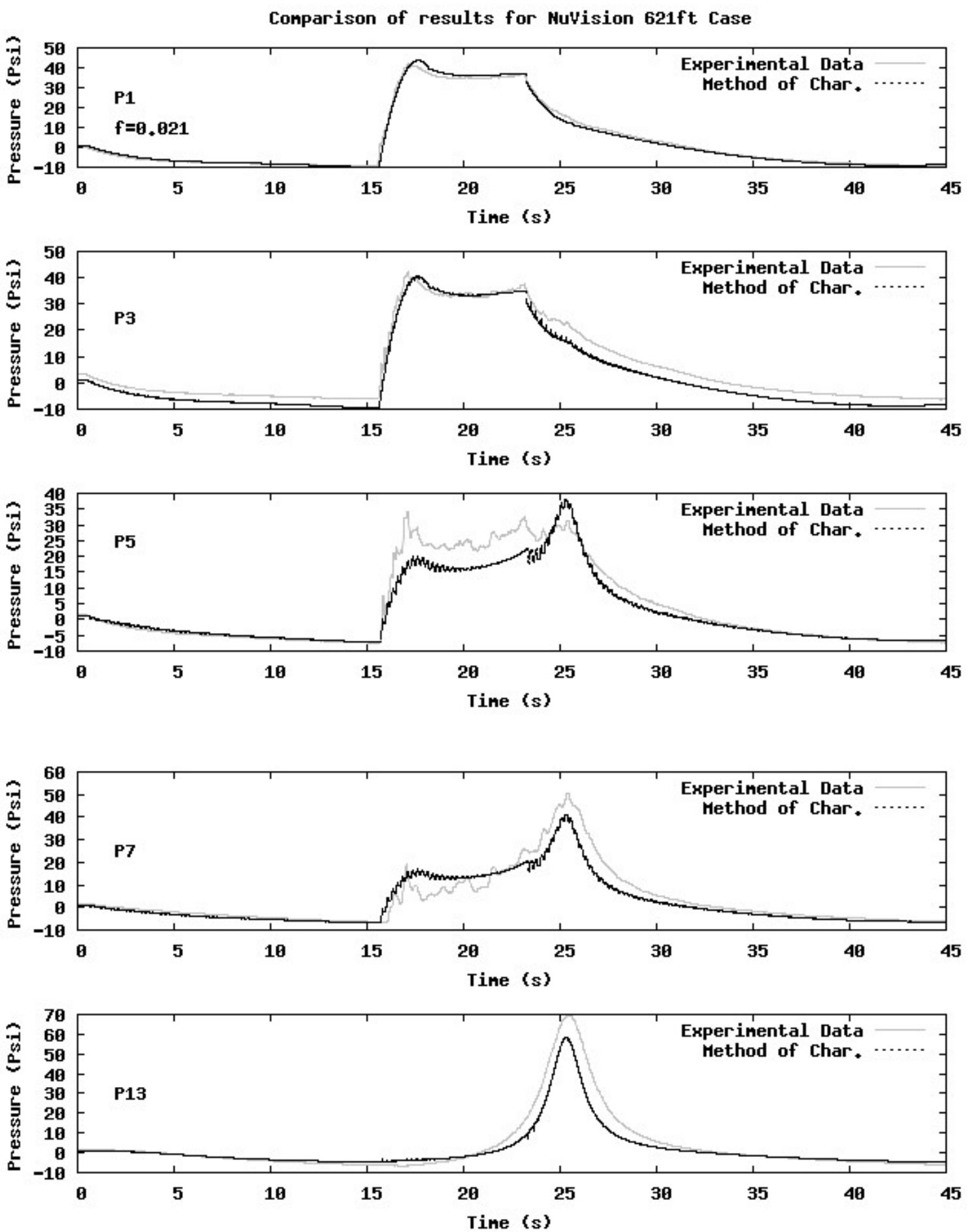

Figure 5-9: Comparision of MOC and Experimental Results for NuVision 621ft Pipeline 

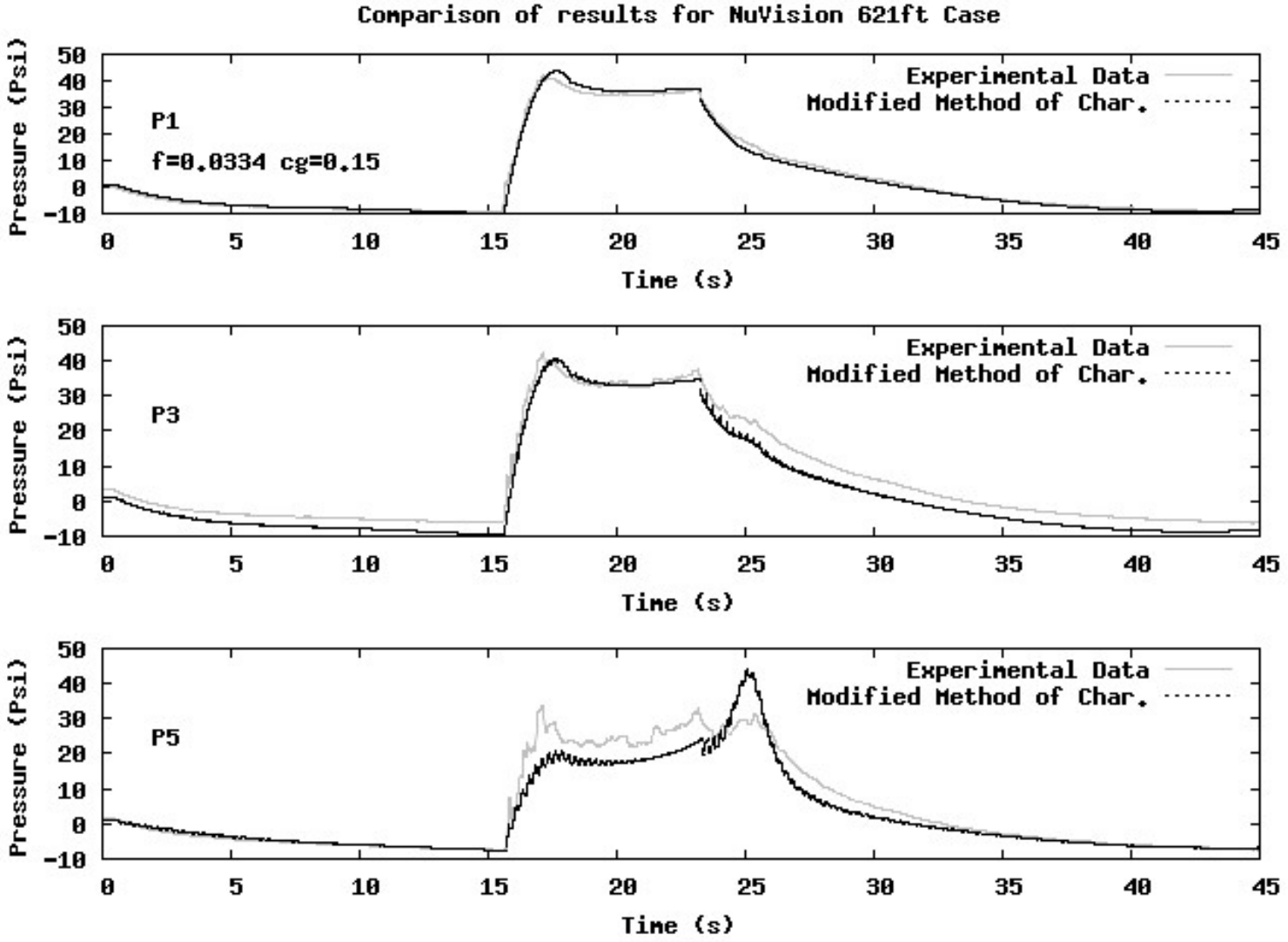

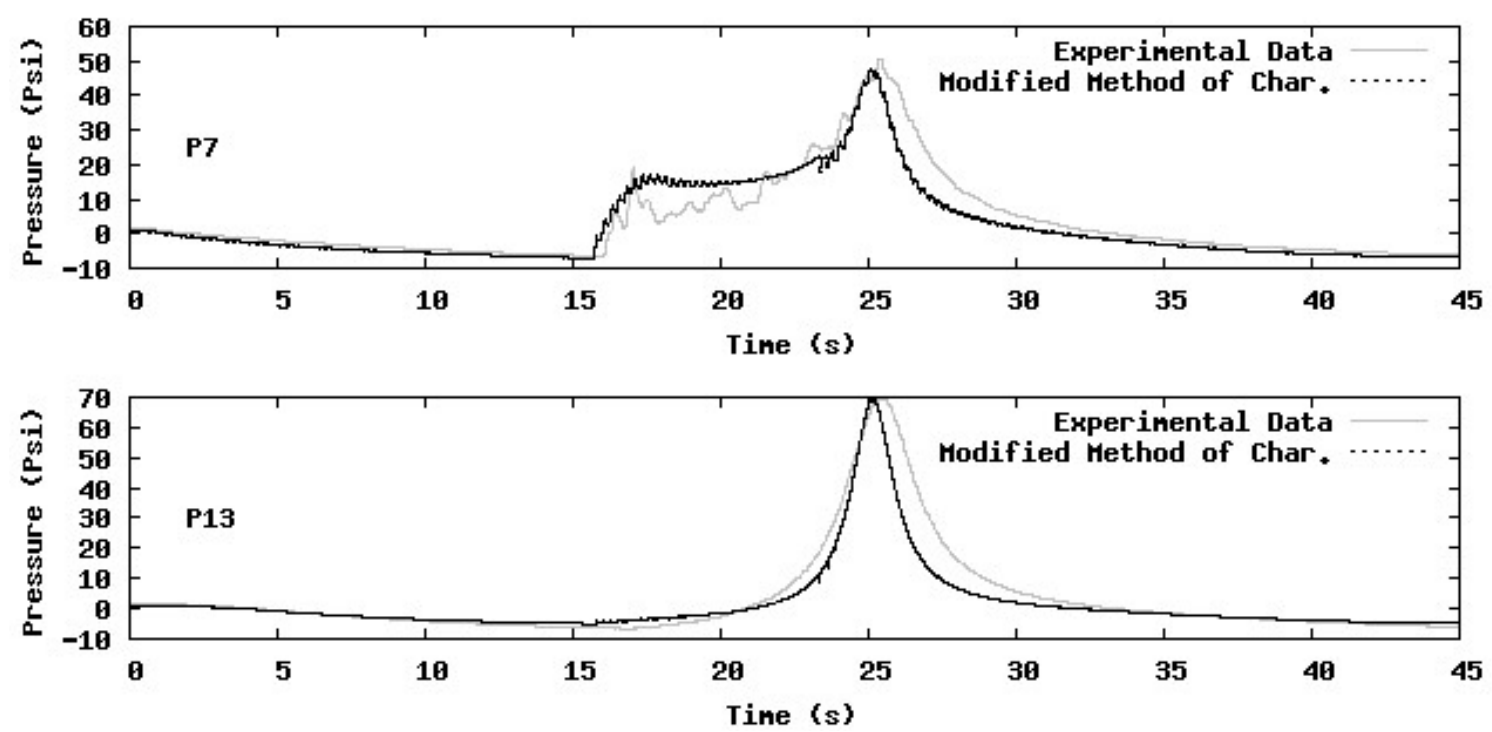

Figure 5-10:Comparision of MMOC and Experimental Results for NuVision 621ft Pipeline

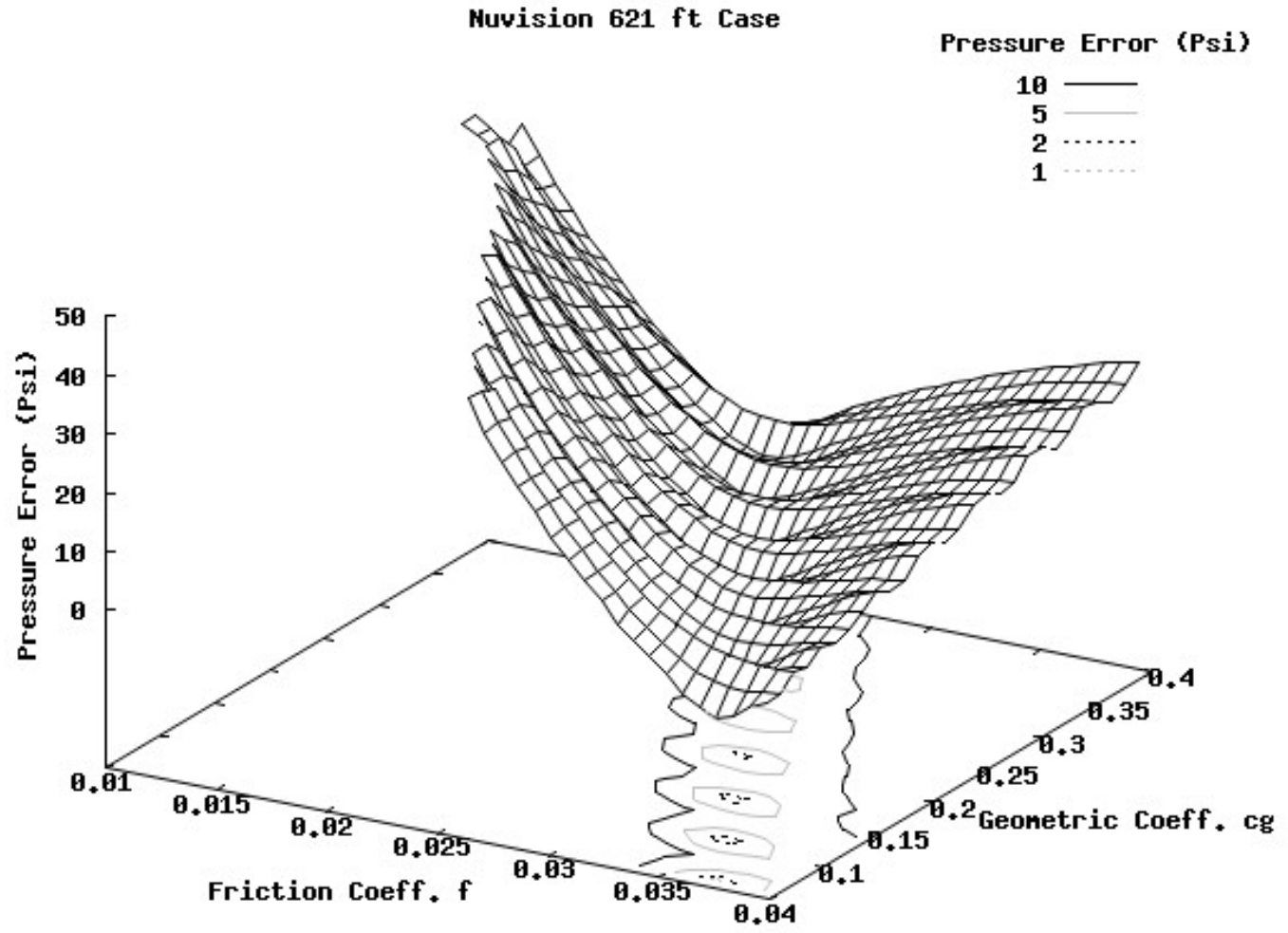

Figure 5-11: Characterization of NuVision 621ft Pipeline by Pressure 


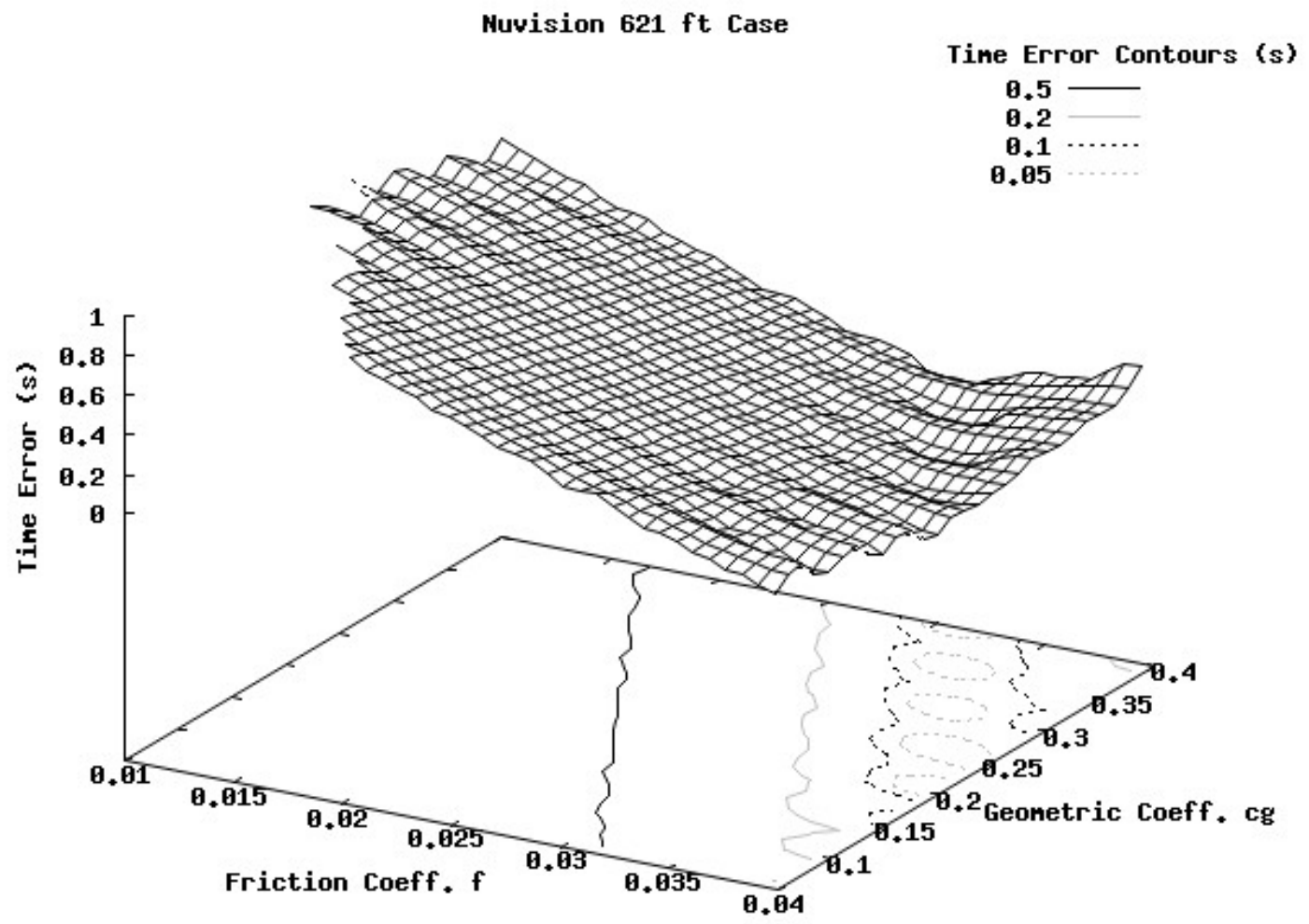

Figure 5-12: Characterization of NuVision 621ft Pipeline by Time Error

\subsubsection{7ft Test Pipeline}

During the technology evaluation of NuVision's Fluidic Wave Action Technology at FIU's ARC the $1797 \mathrm{ft}$ test pipeline was constructed and utilized as shown in Figure 5-13. Measurements from Pressure transducers P1 to P13 positioned as shown in Figure 5-13 were recorded during the evaluation and used as the input to MMOC. A Piecewise least squares fit to the data from P1 is used as the inlet boundary condition in MOC and MMOC. 


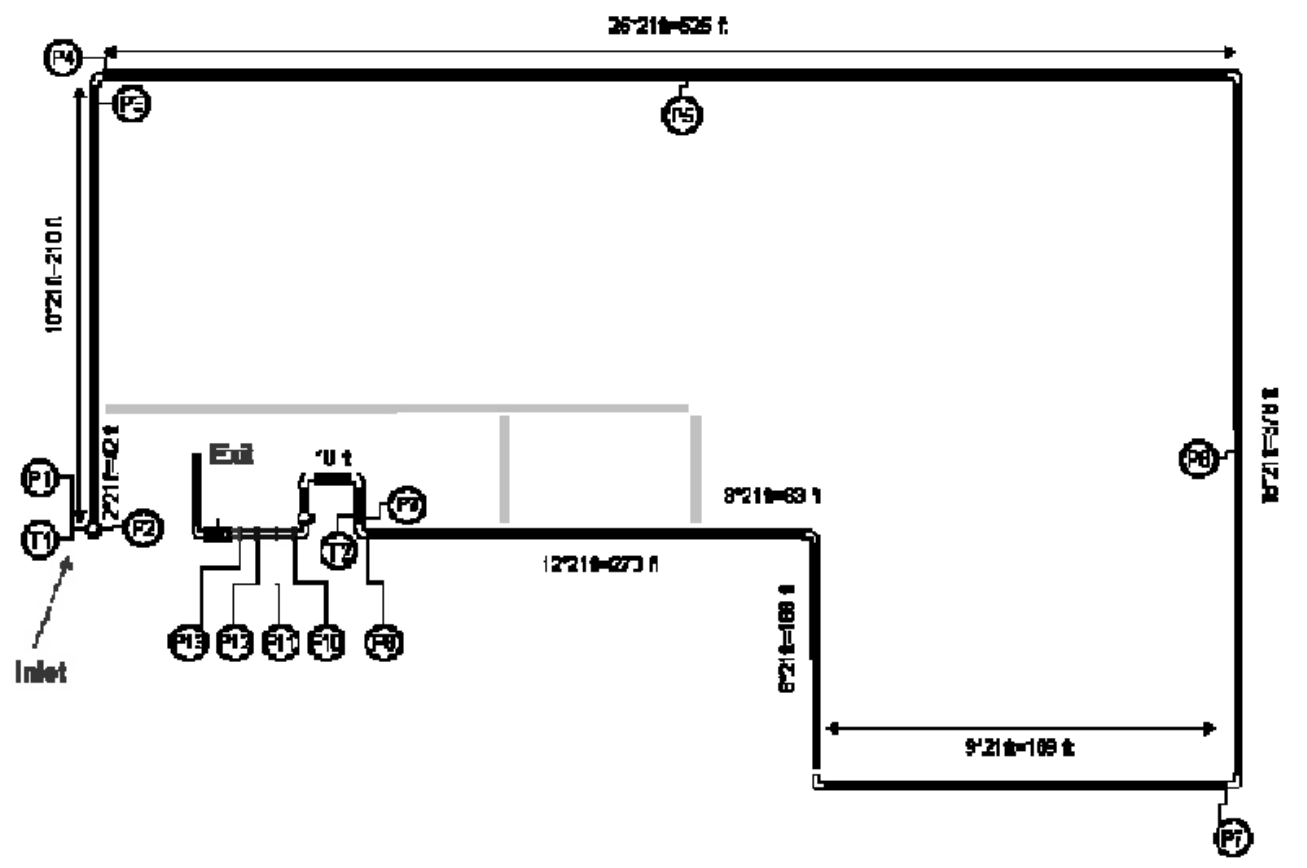

Figure 5-13: Schematic of $1797 \mathrm{ft}$ pipeline [3]

The MMOC model of the $1797 \mathrm{ft}$ pipeline is shown in Figure 5-14. This case differs from both the $285 \mathrm{ft}$ and $621 \mathrm{ft}$ test configurations in three significant ways. First, there are two additional pipes and the two additional $90^{\circ}$ elbows. Second, pipes 1 through 6 are approximately 5 times longer. Third, the pump operation schedule is distinct and has the greatest applied pressure of the three test cases. The experimental data from one trail in the technology evaluation is shown in Figure 5-15 and Figure 5-16. The best results attainable through optimization of the unmodified MOC, are shown in Figure 5-15 and documented in Table 6 alongside the results from MMOC. 


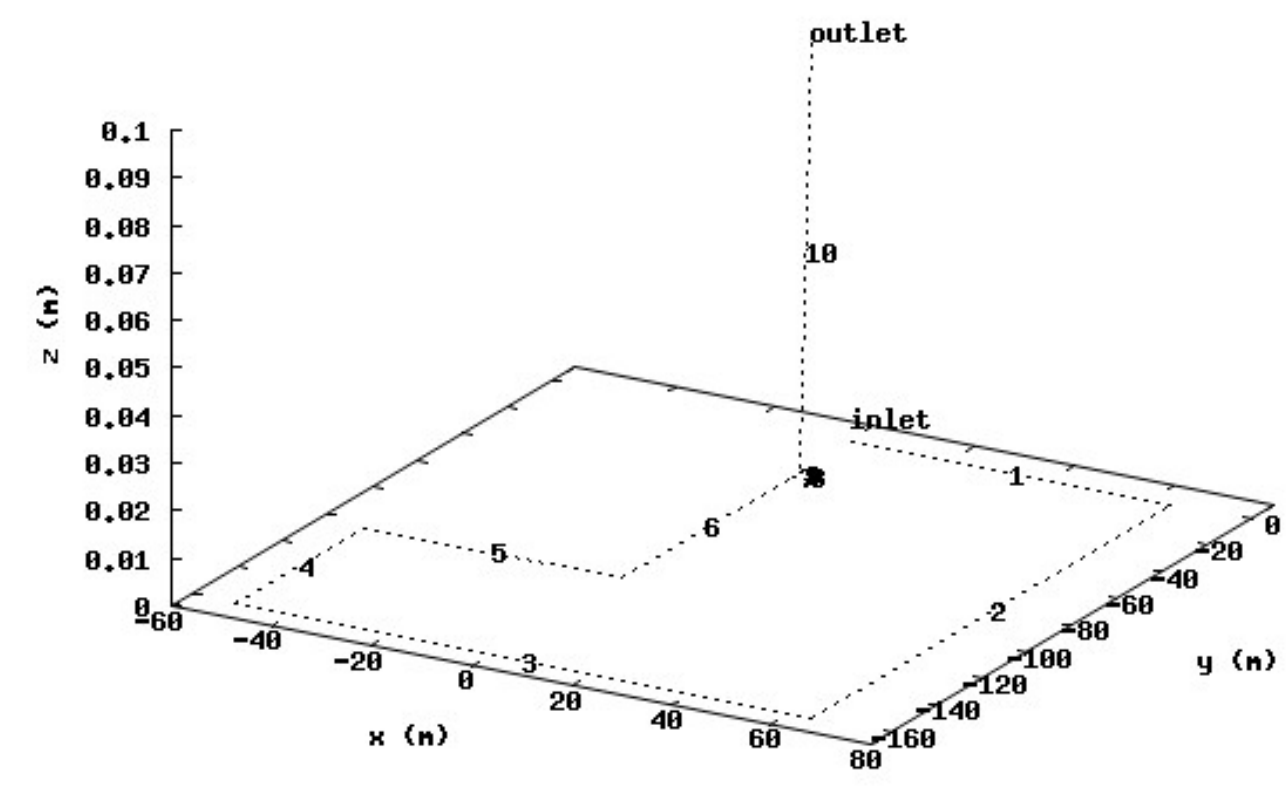

Figure 5-14: Pipeline Model for 1797ft Case

Table 6 shows the increase in accuracy of an order of magnitude by including the proposed geometric loss coefficient to account for the effect of $90^{\circ}$ elbows in the pipeline. Figure 5-15 and Figure 5-16 show that in addition to more accurately predicting the peak pressure the addition of the geometric loss coefficient more accurately captures the transient wave form and the total energy propagating through the pipeline.

Table 6: Comparison of MOC and MMOC for $1797 \mathrm{ft}$ Case

\begin{tabular}{|c|c|c|}
\hline & Pressure Error (Psi) & Time Error (s) \\
\hline Method of Characteristics & & \\
\hline Friction Coefficient, $f=0.021$ & 8.6 & 0.8 \\
\hline Modified Method of Characteristics & & \\
\hline
\end{tabular}




\begin{tabular}{|r|c|c|}
\hline Friction Coefficient, $f=0.0232$ & \multirow{2}{*}{0.2} & 0.03 \\
\cline { 1 - 1 } Geometric Loss Coeff., $c_{g}=0.282$ & & \\
\hline
\end{tabular}

Figure 5-17 displays the difference in pressure between the MMOC simulation and the experimental data. Figure 5-18 displays the difference in time of occurrence of the peak pressure between the MMOC simulation and the experimental data. In both figures the results are shown for the range of friction coefficients and geometric loss coefficients which were available to the genetic algorithm during optimization. Examination of Figure 5-17 reveals the family of values for friction coefficients and geometric loss coefficients which produce accurate pressure predictions from the MMOC simulation. Examination of Figure 5-18 reveals the limited islands of values for friction coefficients and geometric loss coefficients which produce accurate time of peak pressure predictions from the MMOC simulation. It is notable that the families of values for accurate pressure and time predictions overlap less substantially for this test pipeline than they do for the $285 \mathrm{ft}$ pipeline or for the $621 \mathrm{ft}$ pipeline. 

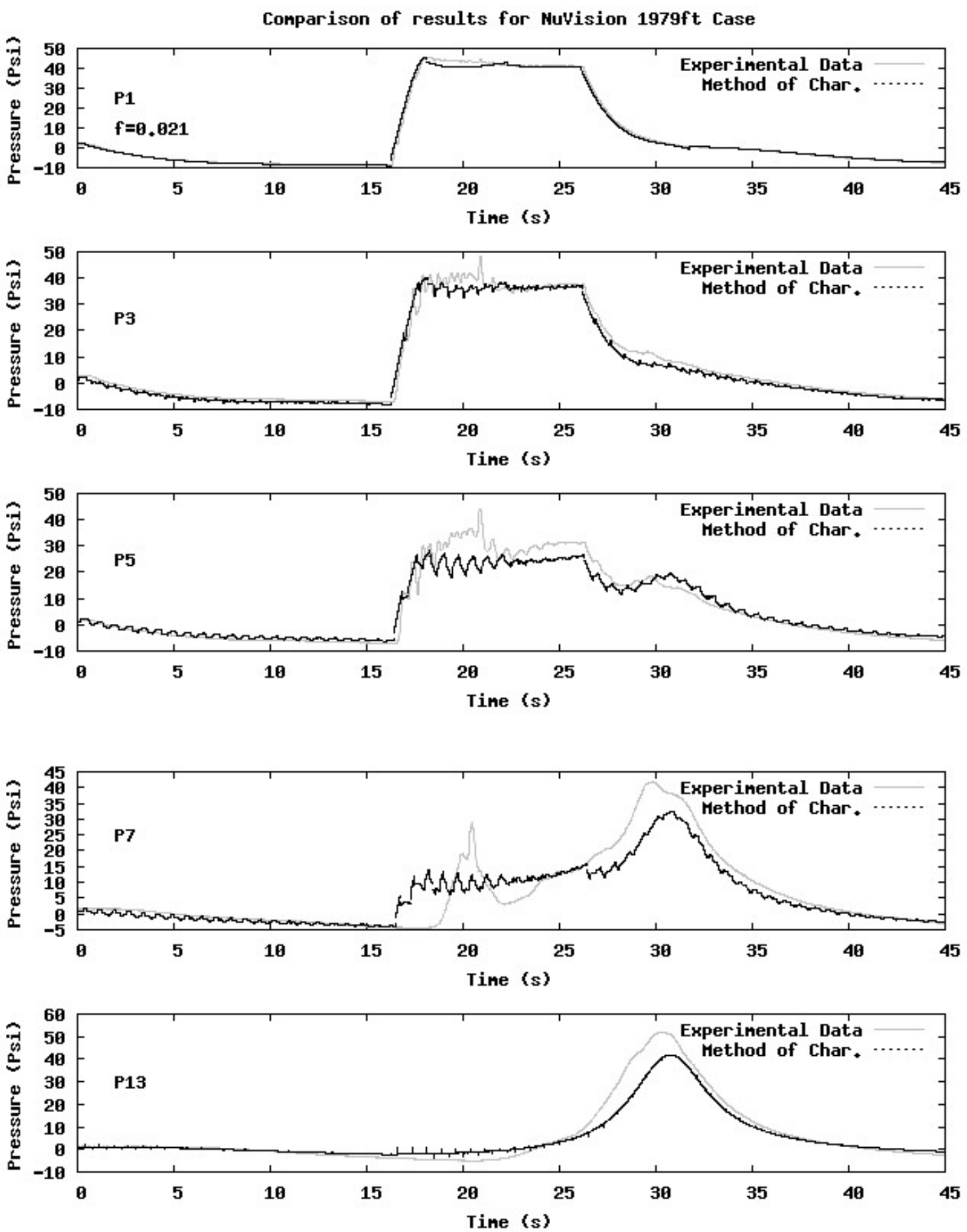

Figure 5-15:Comparision of MOC and Experimental Results for NuVision 1797ft Pipeline 
Conparison of results for Muvision $1979 \mathrm{ft}$ Case
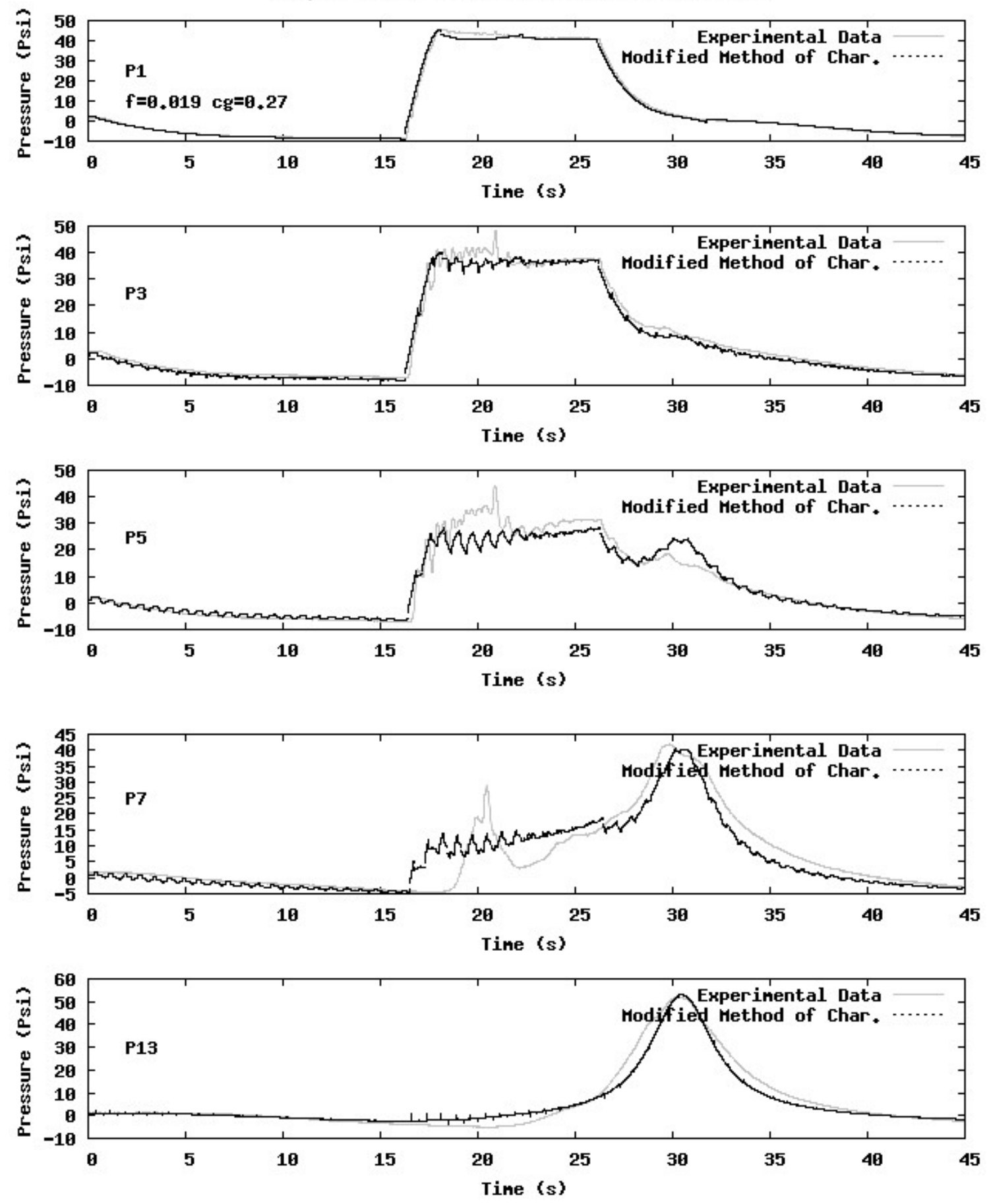

Figure 5-16:Comparision of MMOC and Experimental Results for NuVision 1797ft Pipeline 


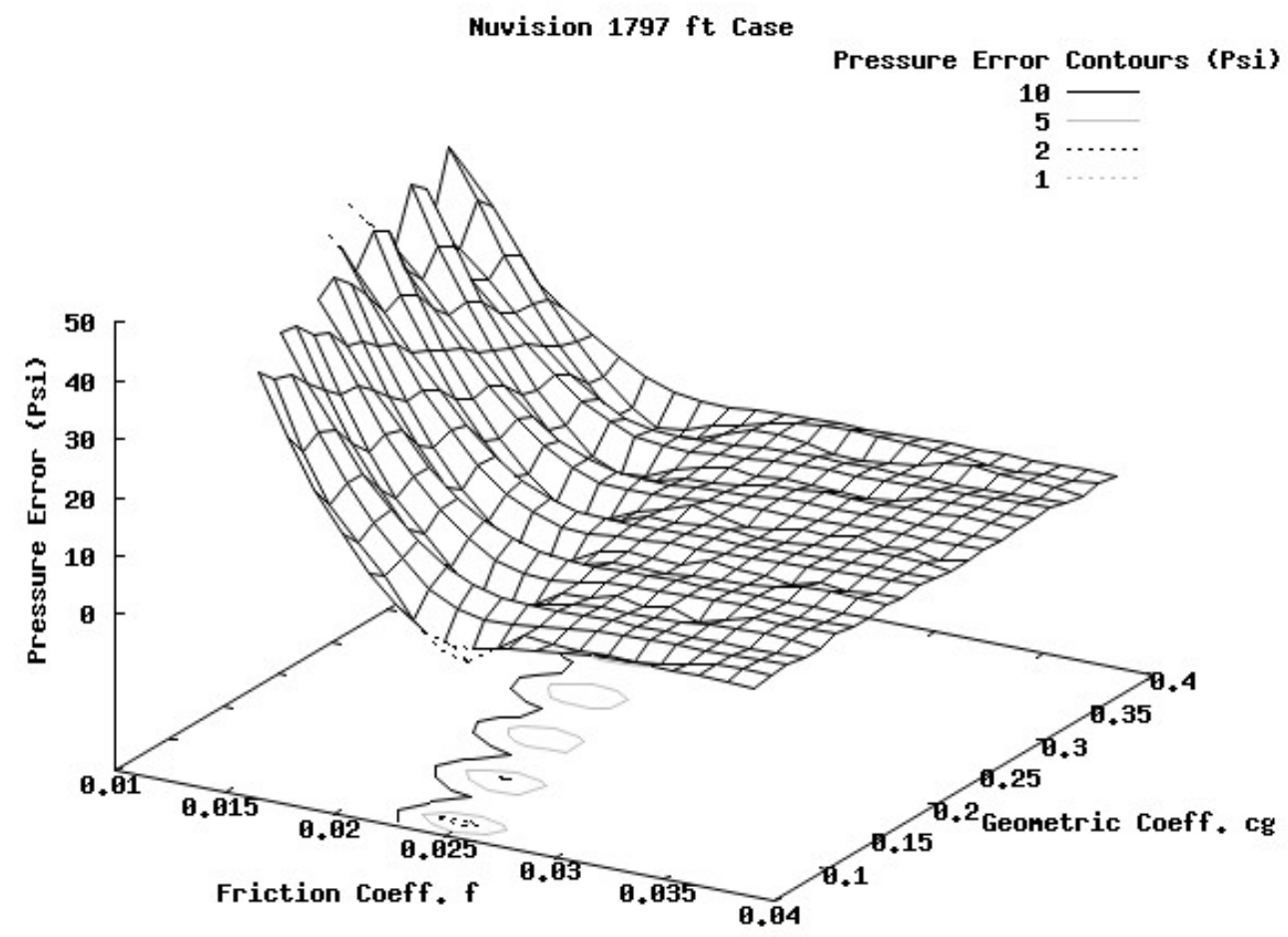

Figure 5-17: Characterization of NuVision 1797ft Pipeline by Pressure Error

Muvision $1797 \mathrm{ft}$ Case

Tine Error Contours (s)
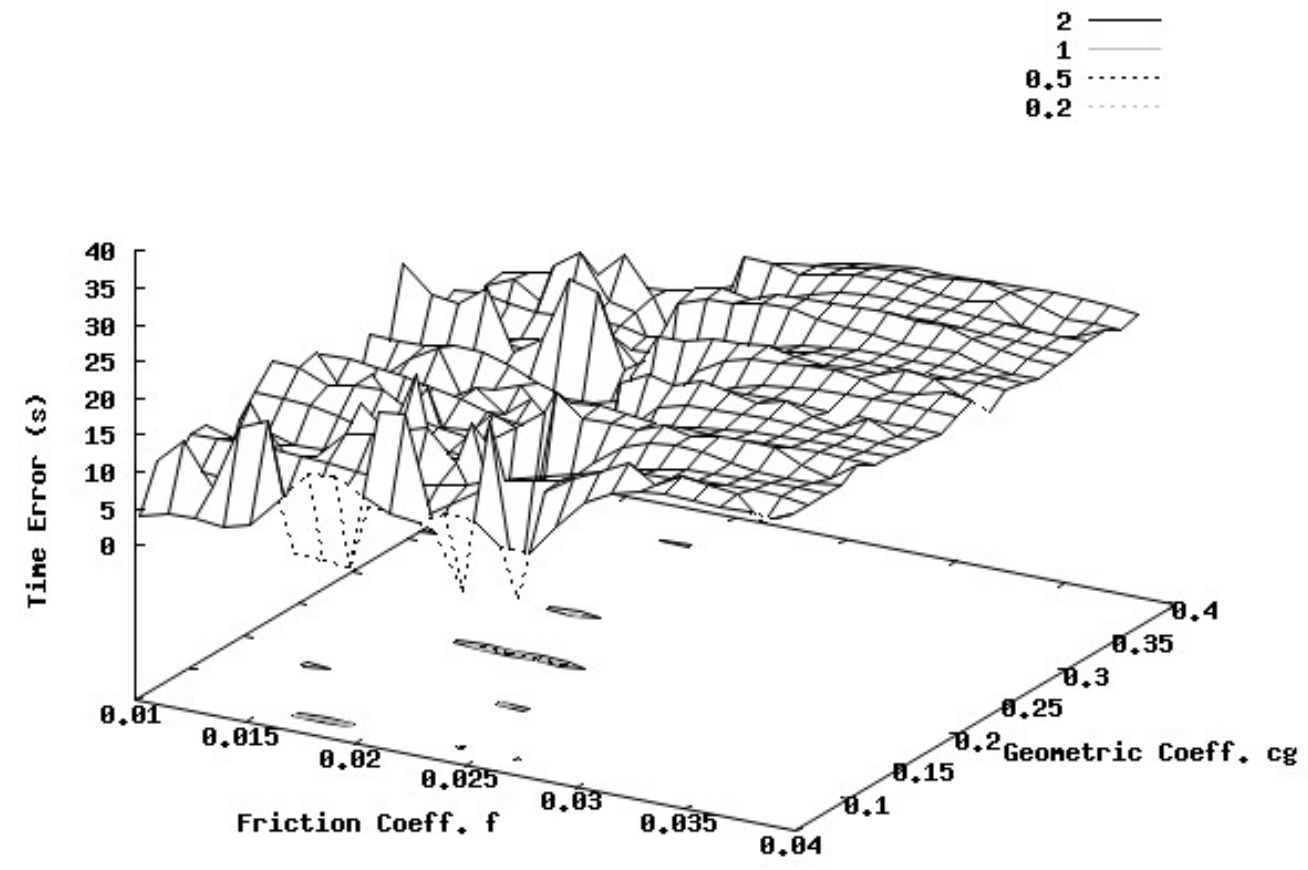

Figure 5-18: Characterization of NuVision 1797ft Pipeline by Time Error 


\subsection{Web Based Implementation}

PHP was successfully utilized to create a password protected web interface for MMOC. The visual organization made possible by the web form greatly facilitates the accurate entry of the input parameters. The error checking provided by the form improves the utility of MMOC by pre-empting the need for time consuming trouble shooting.

\begin{tabular}{|c|c|c|c|c|c|c|c|c|}
\hline \multicolumn{2}{|c|}{$\begin{array}{l}\text { Case Name NuVision285 } \\
1\end{array}$} & \# of pipes 8 & \multicolumn{2}{|c|}{ Friction factor 0.021} & \multicolumn{2}{|c|}{ Elbow Loss Coefficient 0.3} & \\
\hline & \multicolumn{3}{|c|}{ Dimentions } & \multicolumn{2}{|c|}{ Pipe Material } & \multicolumn{3}{|c|}{ Position } \\
\hline & $\begin{array}{l}\text { Length } \\
(\mathrm{m})\end{array}$ & $\begin{array}{l}\text { Diameter } \\
(\mathrm{m})\end{array}$ & $\begin{array}{l}\text { Wall Thk. } \\
\text { (m) }\end{array}$ & $\begin{array}{l}\text { Elastic Modulus } \\
\left(\mathrm{N} / \mathrm{m}^{\wedge} 2\right)\end{array}$ & Poison Ratio & $\begin{array}{l}\text { Orientation } \\
\text { (degrees) }\end{array}$ & & $\begin{array}{l}\text { Inclination } \\
\text { (degrees) }\end{array}$ \\
\hline Pipe 1 & 12.8016 & 0.0762 & 0.0063 & $200 \mathrm{e} 9$ & 0.3 & 0 & 0 & \\
\hline Pipe 2 & 32.004 & 0.0762 & 0.0063 & $200 \mathrm{e} 9$ & 0.3 & 270 & 0 & \\
\hline Pipe 3 & 12.8016 & 0.0762 & 0.0063 & $200 \mathrm{e} 9$ & 0.3 & 180 & 0 & \\
\hline Pipe 4 & 12.8016 & 0.0762 & 0.0063 & $200 \mathrm{e} 9$ & 0.3 & 90 & 0 & \\
\hline Pipe 5 & 3.0248 & 0.0762 & 0.0063 & $200 \mathrm{e} 9$ & 0.3 & 0 & 0 & \\
\hline Pipe 6 & 3.048 & 0.0762 & 0.0063 & $200 \mathrm{e} 9$ & 0.3 & 90 & 0 & \\
\hline Pipe 7 & 3.048 & 0.0762 & 0.0063 & $200 \mathrm{e} 9$ & 0.3 & 180 & 0 & \\
\hline Pipe 8 & 5.1816 & 0.0762 & 0.0063 & $200 \mathrm{e} 9$ & 0.3 & 90 & 1 & \\
\hline
\end{tabular}

Figure 5-19: Web Form for Pipeline configuration

Figure 5-19 and Figure 5-20 show the web forms for the pipeline configuration and boundary conditions for MMOC. Figure 5-21 shows a typical input file which by comparison is far less readable and offers no error checking for inputs by the users. 


\title{
Boundary Conditions
}

\author{
Inlet \\ Constant Reservoir \\ Variable Reservoir $\nabla$ \\ Outlet \\ Valve \\ Air Pocket $\square$ \\ Dead End
}

Simulate Transients $\sqrt{\square}$

Submit

\section{Figure 5-20: Web Form for Boundary Condition Selection}

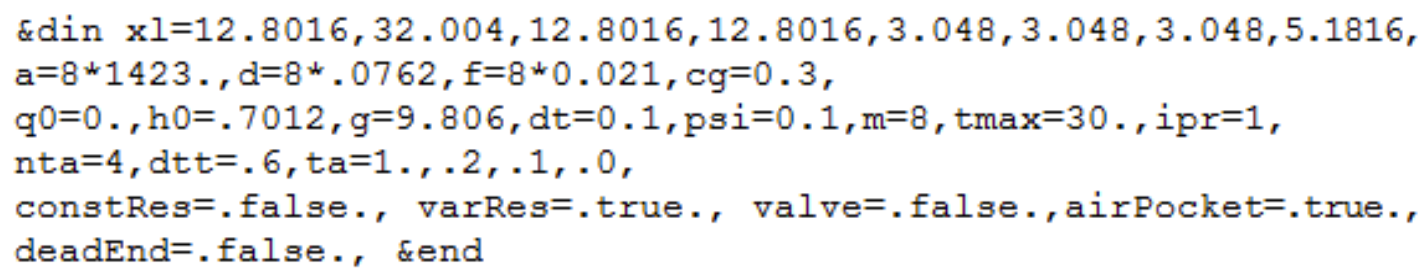

Figure 5-21: Input file

Figure 5-22 displays typical output that is generated by the web interface to MMOC in seconds following the submission of a MMOC job to Tesla. 


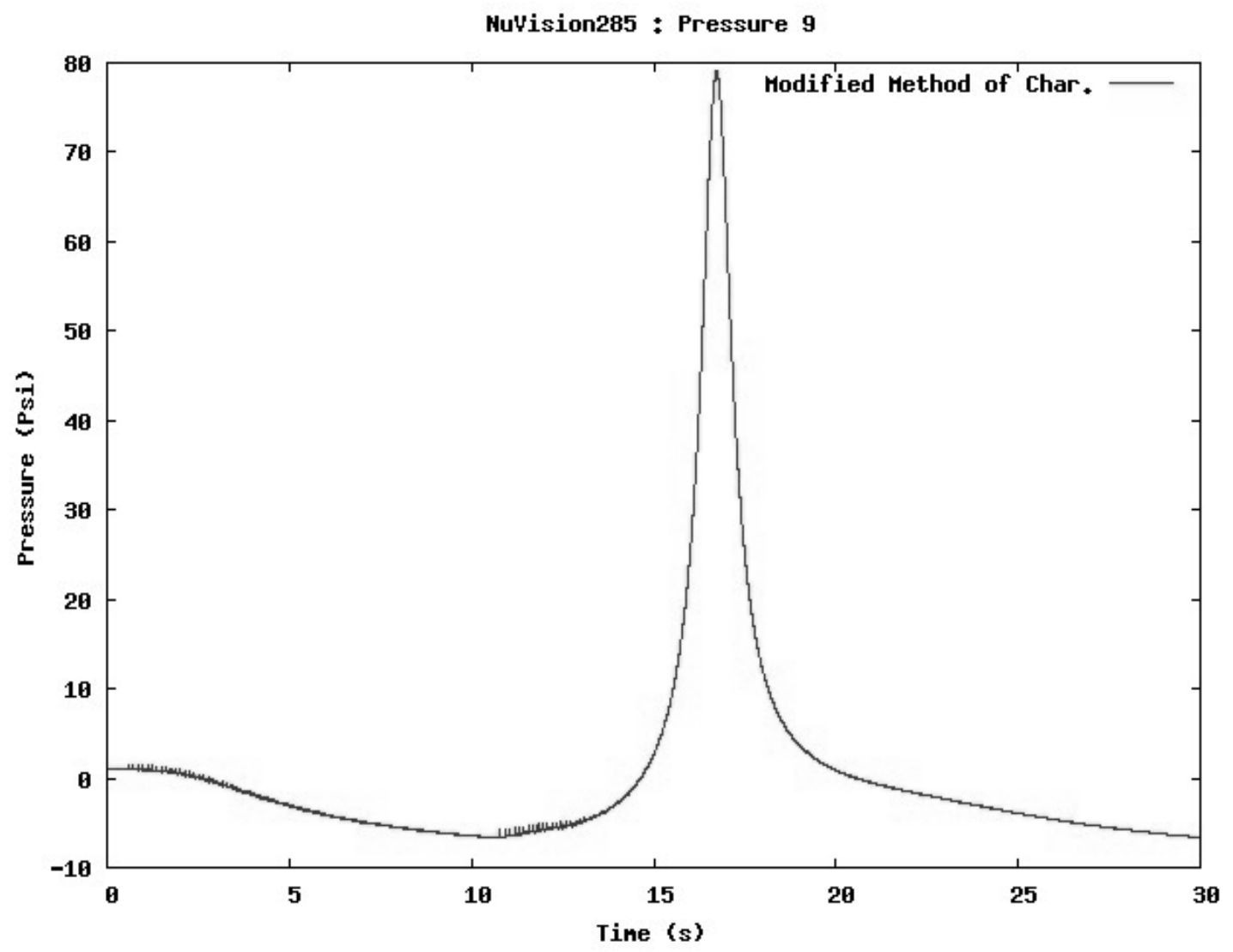

Figure 5-22: Sample Web MMOC Plot

\subsection{Optimization of the Inlet Hydraulic Unit Operation Schedule}

After a pipeline has been characterized as detailed in section 5.1 the operating schedule of the pump at the inlet can be optimized with the goal of producing a peak pressure at a specified location in the pipeline. 


\subsubsection{5ft Test Pipeline}

The objective of the following pump operation optimization for the $285 \mathrm{ft}$ pipeline is to produce a peak pressure of 73 Psi at pressure sensor P13 at the end of pipe segment 8. Figure 5-23 and Figure 5-24 display the pressure error and time error respectively for the ranges of hold time and applied pressure detailed in Table 2.

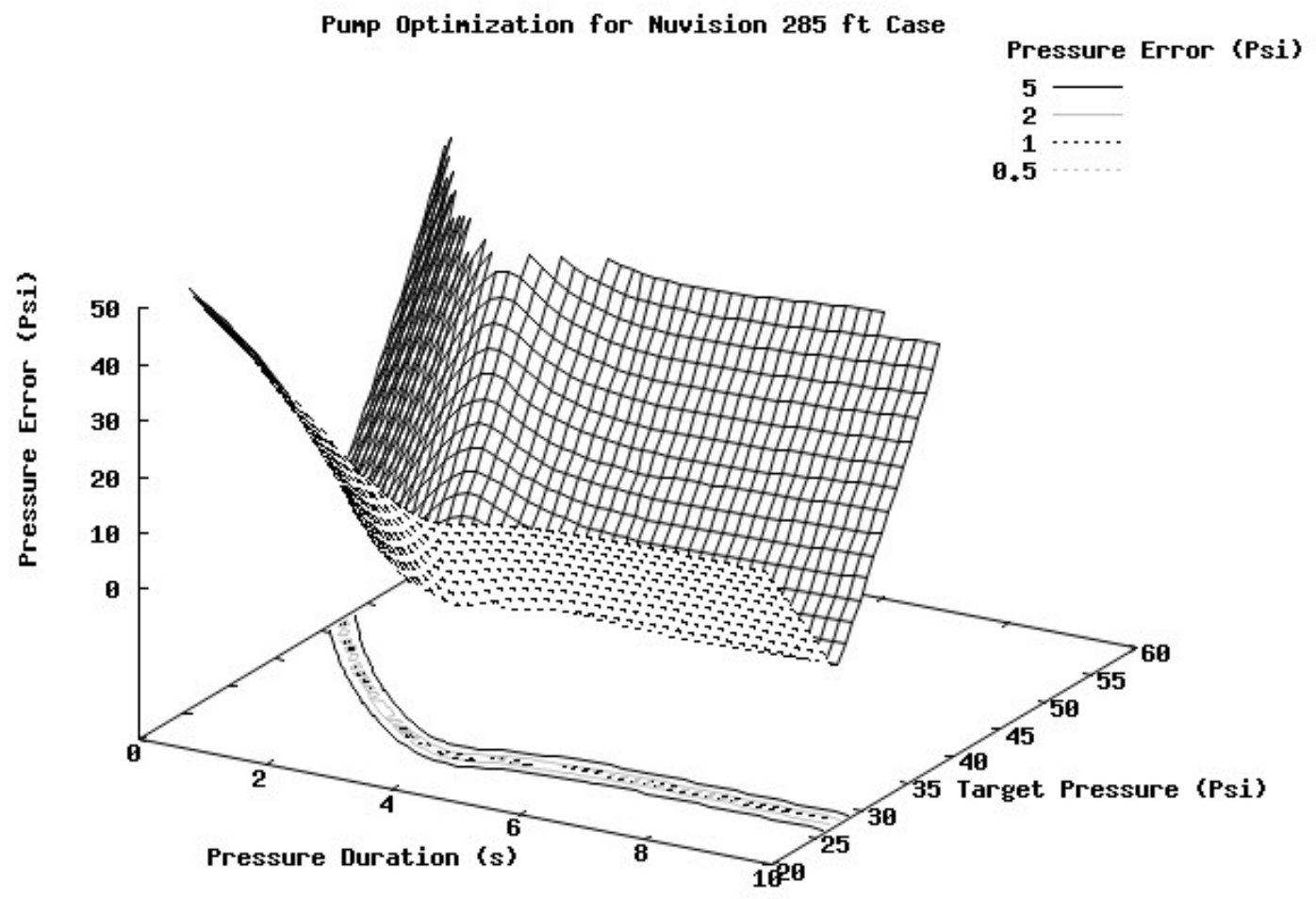

Figure 5-23: Pump Operation Optimization for NuVision 285ft Pipeline by Pressure Error 


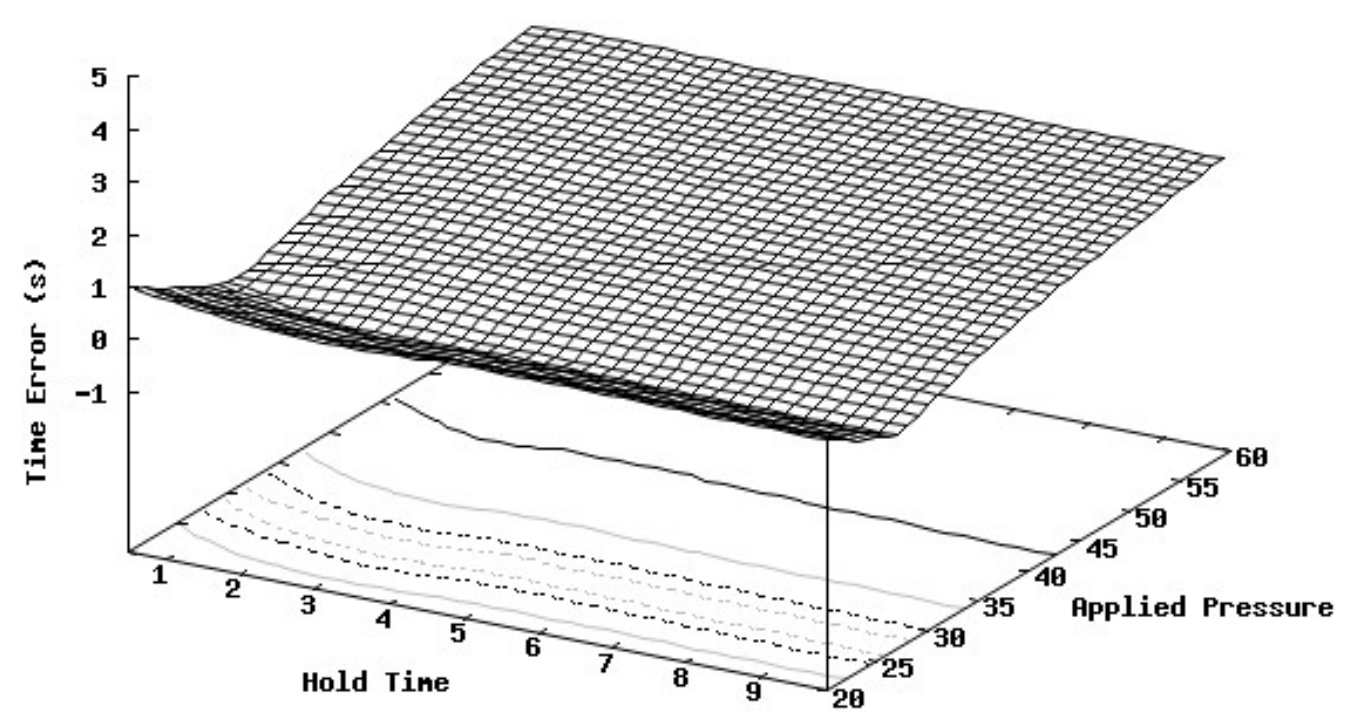

Figure 5-24: Pump Operation Optimization for NuVision 285ft Pipeline by Time Error

Examination of Figure 5-23 and Figure 5-24 reveals that it is possible to achieve peak pressures of 73 Psi at pressure sensor P13 for applied pressures between 25 and 40 psi by varying the duration the pressure is applied by the pump.

Figure 5-25, Figure 5-26 and Figure 5-27 display the pressure time histories for the initial, an intermediate and the final sets of pump optimization parameters as specified in Table 7.

The results show that the pump operation optimization by a genetic algorithm can provide a specific recommendation for a pumping schedule to achieve a desired pressure 
at a particular position in the $285 \mathrm{ft}$ pipeline. Optimization also reveals the response of the system over the range of the input parameters.

Table 7: Pump Operation Optimization Summary for $285 \mathrm{ft}$ Case

\begin{tabular}{|l|l|l|l|l|}
\hline & Hold Time (s) & $\begin{array}{c}\text { Applied Pressure } \\
(\text { Psi) }\end{array}$ & $\begin{array}{c}\text { Pressure Error } \\
(\mathrm{Psi})\end{array}$ & $\begin{array}{c}\text { Time Error } \\
(\mathrm{s})\end{array}$ \\
\hline Initial & 5.1 & 25.6 & 6.07 & 0.2 \\
\hline Interim & 3.1 & 45.6 & 86.92 & 1.1 \\
\hline Final & 1.3 & 35.2 & 0.14 & 0.5 \\
\hline
\end{tabular}



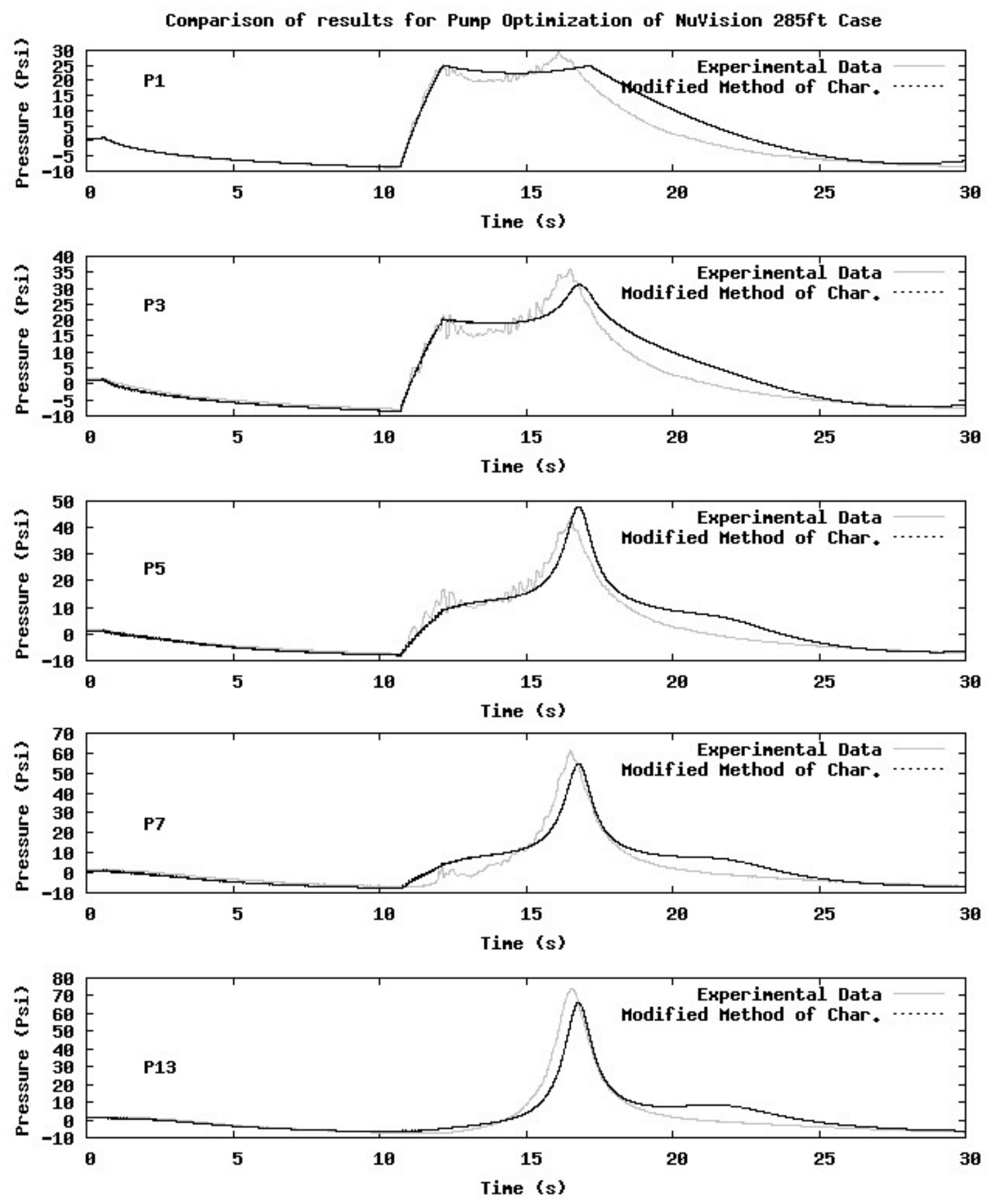

Figure 5-25: Initial Pump Optimization Results for 285ft Case 
Conparison of results for Punp Optinization of Nuvision 285ft Case
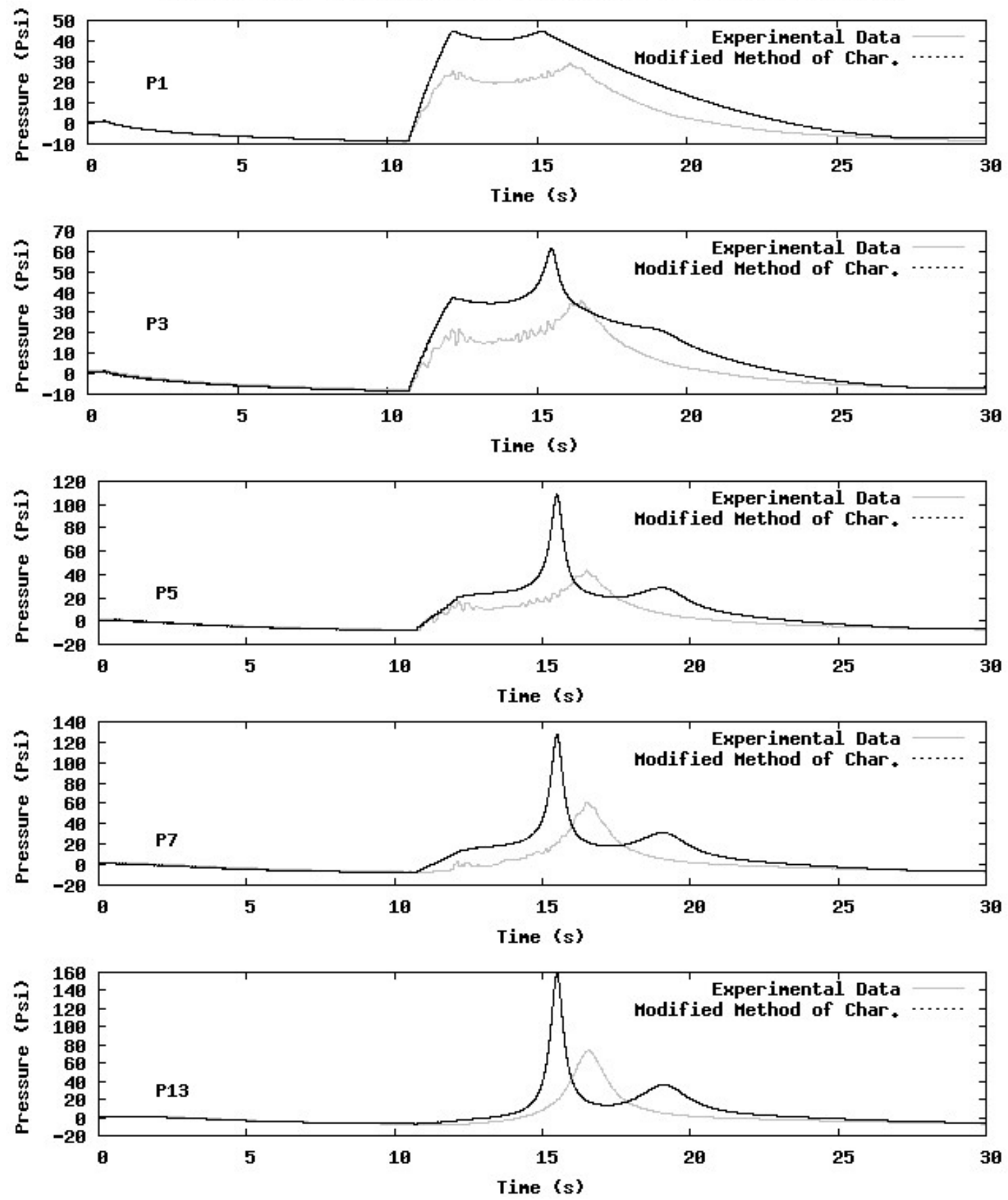

Figure 5-26: Interim Pump Optimization Results for 285ft Case 

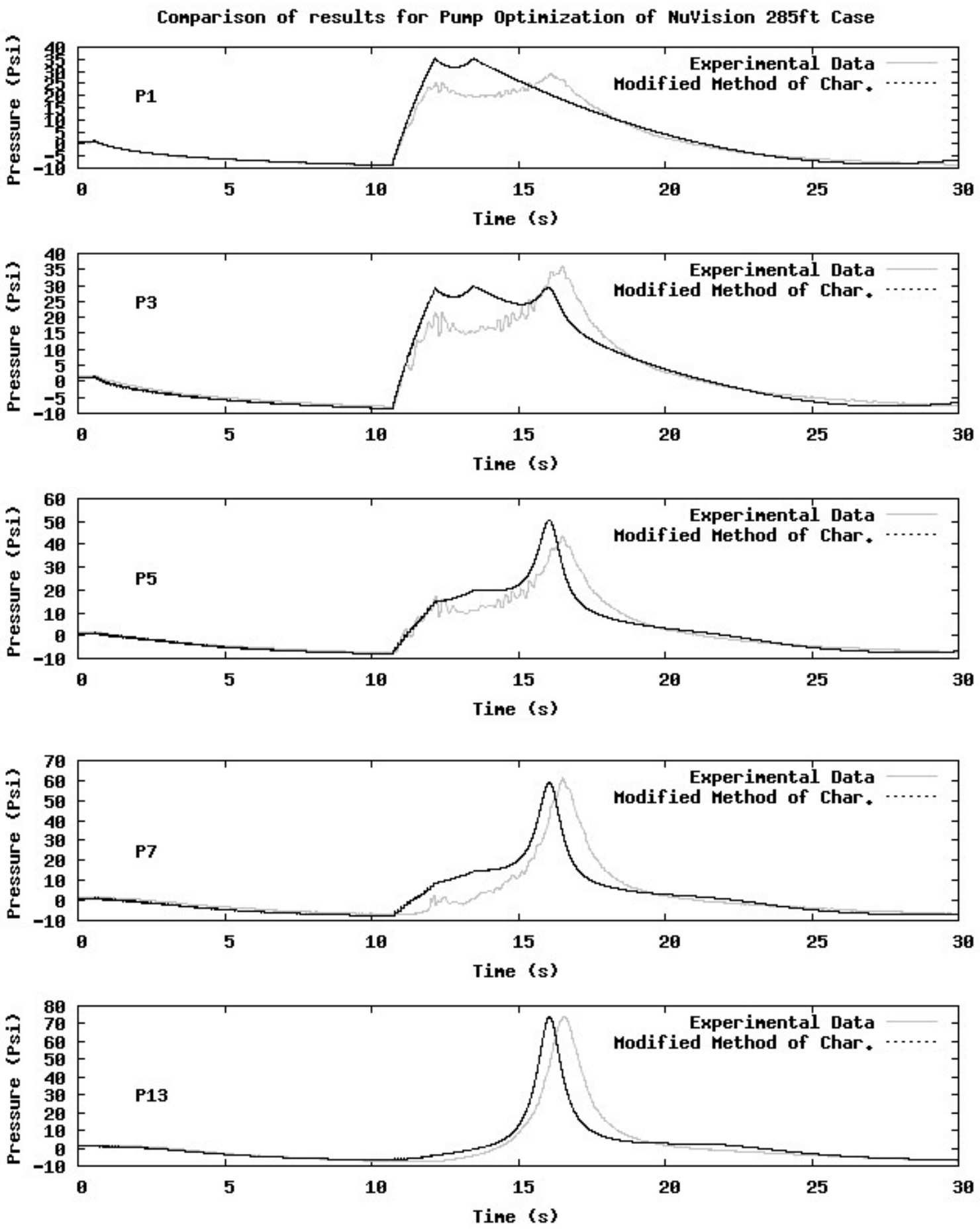

Figure 5-27: Final Pump Optimization Results for $285 \mathrm{ft}$ Case 


\subsubsection{1ft Test Pipeline}

The objective of the following pump operation optimization for the $621 \mathrm{ft}$ pipeline is to produce a peak pressure of 70 Psi at pressure sensor P13 at the end of pipe segment 8. Figure 5-28 and Figure 5-29 display the pressure error and time error respectively for the ranges of hold time and applied pressure detailed in Table 2.

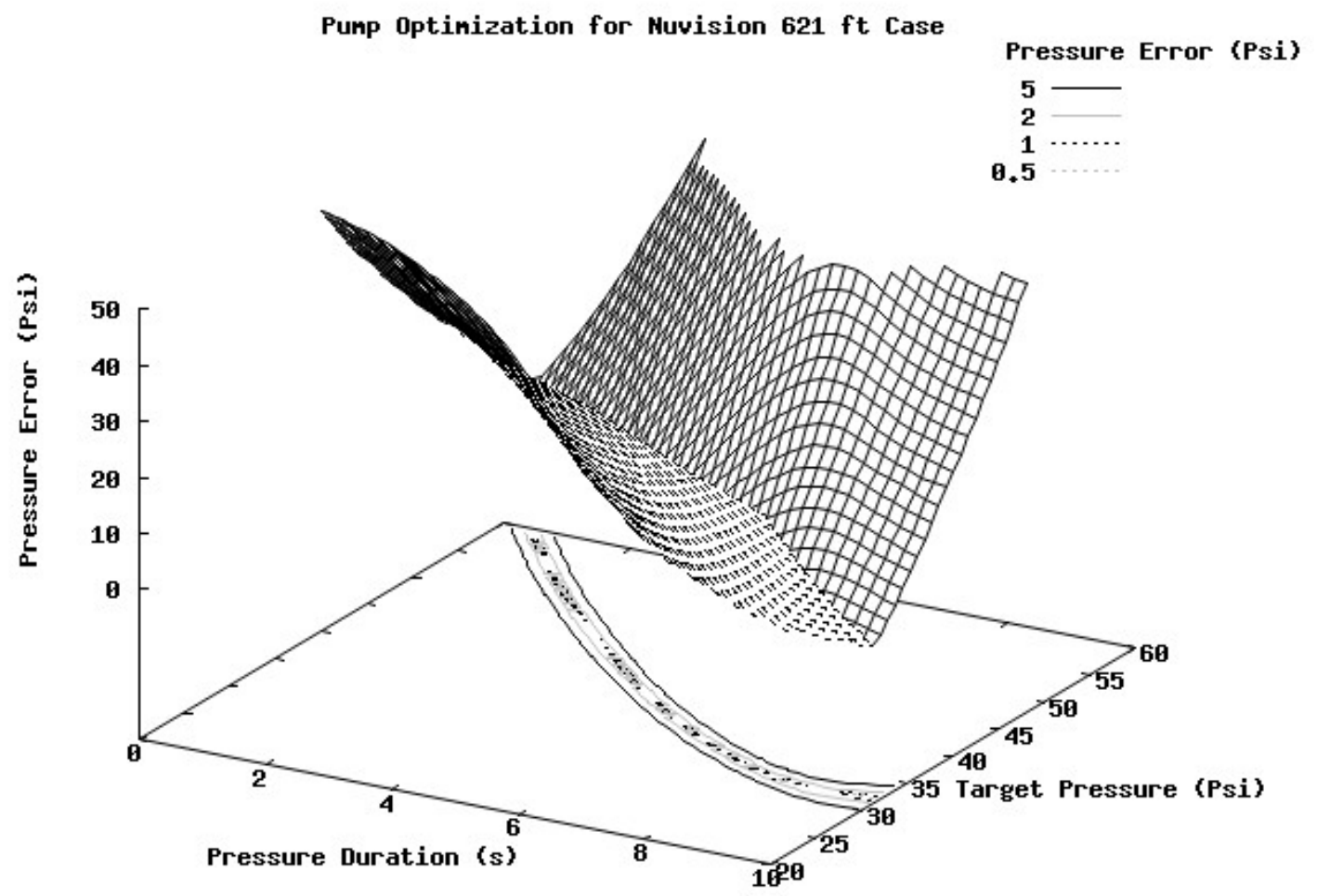

Figure 5-28: Pump Operation Optimization for NuVision 621ft Pipeline by Pressure Error 


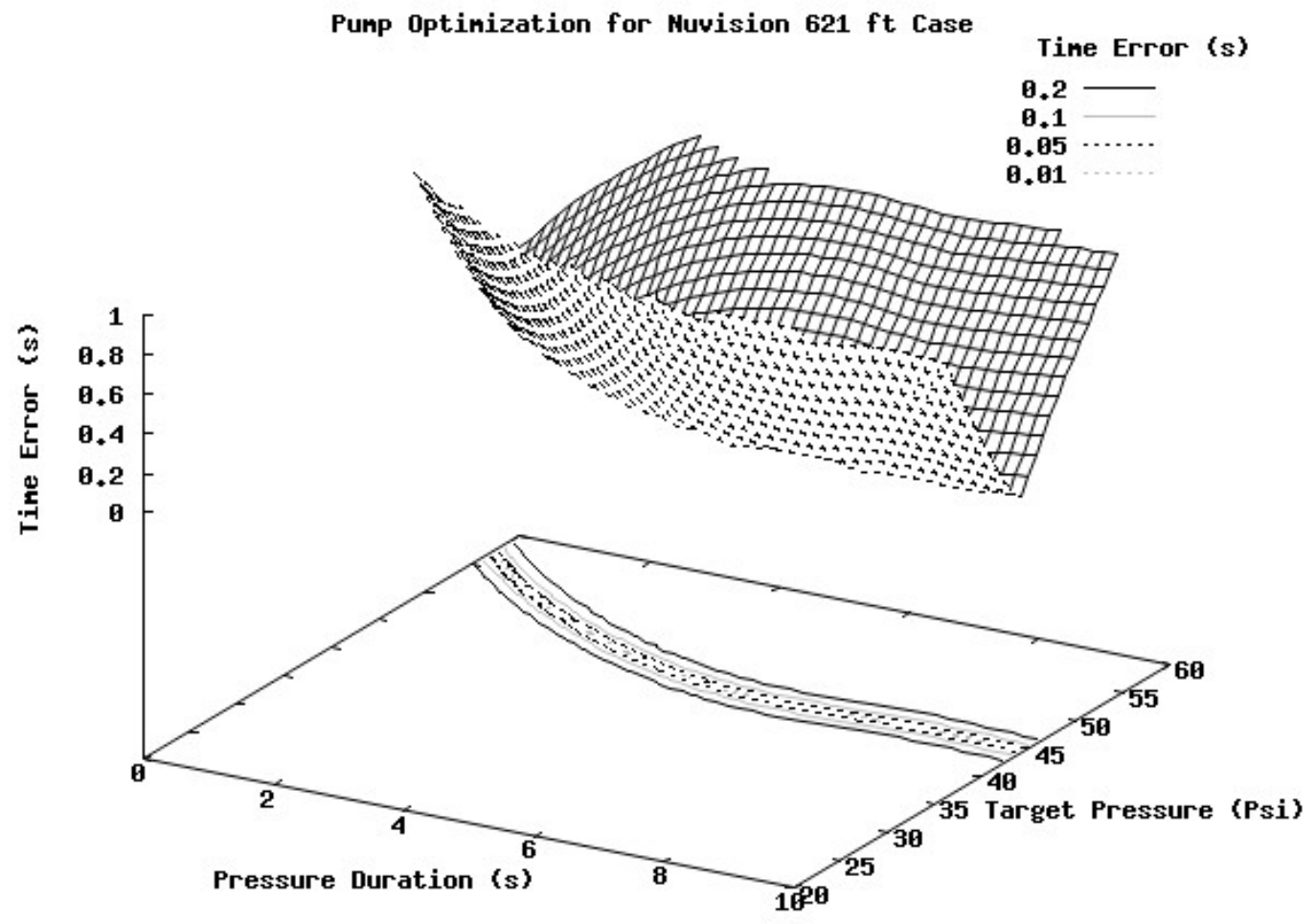

Figure 5-29: Pump Operation Optimization for NuVision 621ft Pipeline by Time Error

Examination of Figure 5-28 and Figure 5-29 reveals that it is possible to achieve peak pressures of 70 Psi at pressure sensor P13 for applied pressures between 30 and 60 psi by varying the duration the pressure is applied by the pump.

Figure 5-30, Figure 5-31 and Figure 5-32 display the pressure time histories for the initial, an intermediate and the final sets of pump optimization parameters as specified in Table 8.

The results show that the pump operation optimization by a genetic algorithm can provide a specific recommendation for a pumping schedule to achieve a desired pressure 
at a particular position in the $621 \mathrm{ft}$ pipeline. Optimization also reveals the response of the system over the range of the input parameters.

Table 8: Pump Operation Optimization Summary for 621ft Case

\begin{tabular}{|l|l|l|l|l|}
\hline & $\begin{array}{c}\text { Hold Time } \\
(\mathrm{s})\end{array}$ & $\begin{array}{c}\text { Applied Pressure } \\
(\mathrm{Psi})\end{array}$ & $\begin{array}{c}\text { Pressure Error } \\
(\mathrm{Psi})\end{array}$ & $\begin{array}{c}\text { Time Error } \\
(\mathrm{s})\end{array}$ \\
\hline Initial & 2.1 & 30.4 & 51.97 & 2.7 \\
\hline Interim & 5.3 & 50.4 & 47.95 & 0.53 \\
\hline Final & 4.5 & 42.4 & 0.001 & 0.37 \\
\hline
\end{tabular}



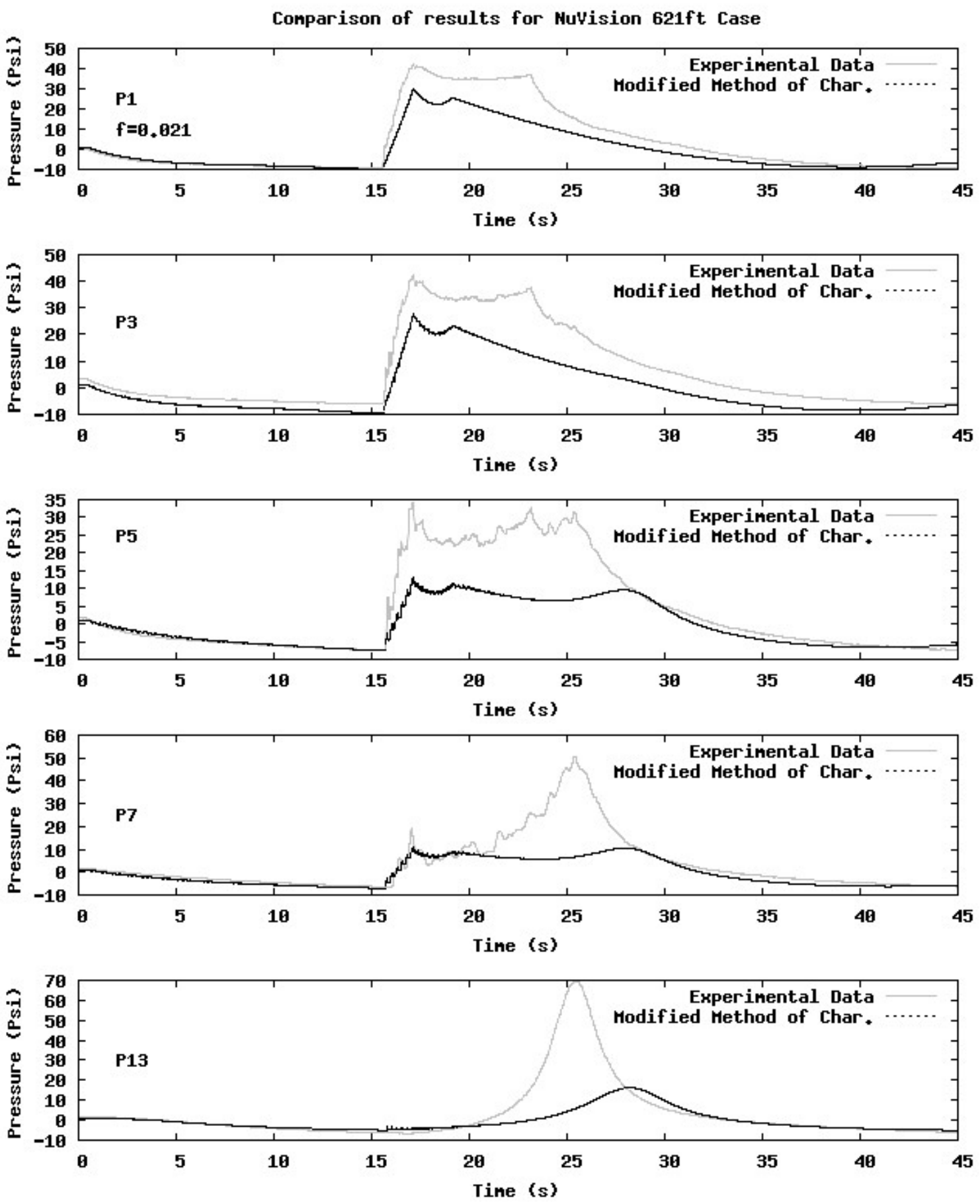

Figure 5-30: Initial Pump Optimization Results for 621ft Case 
Comparison of results for MuVision 621ft Case
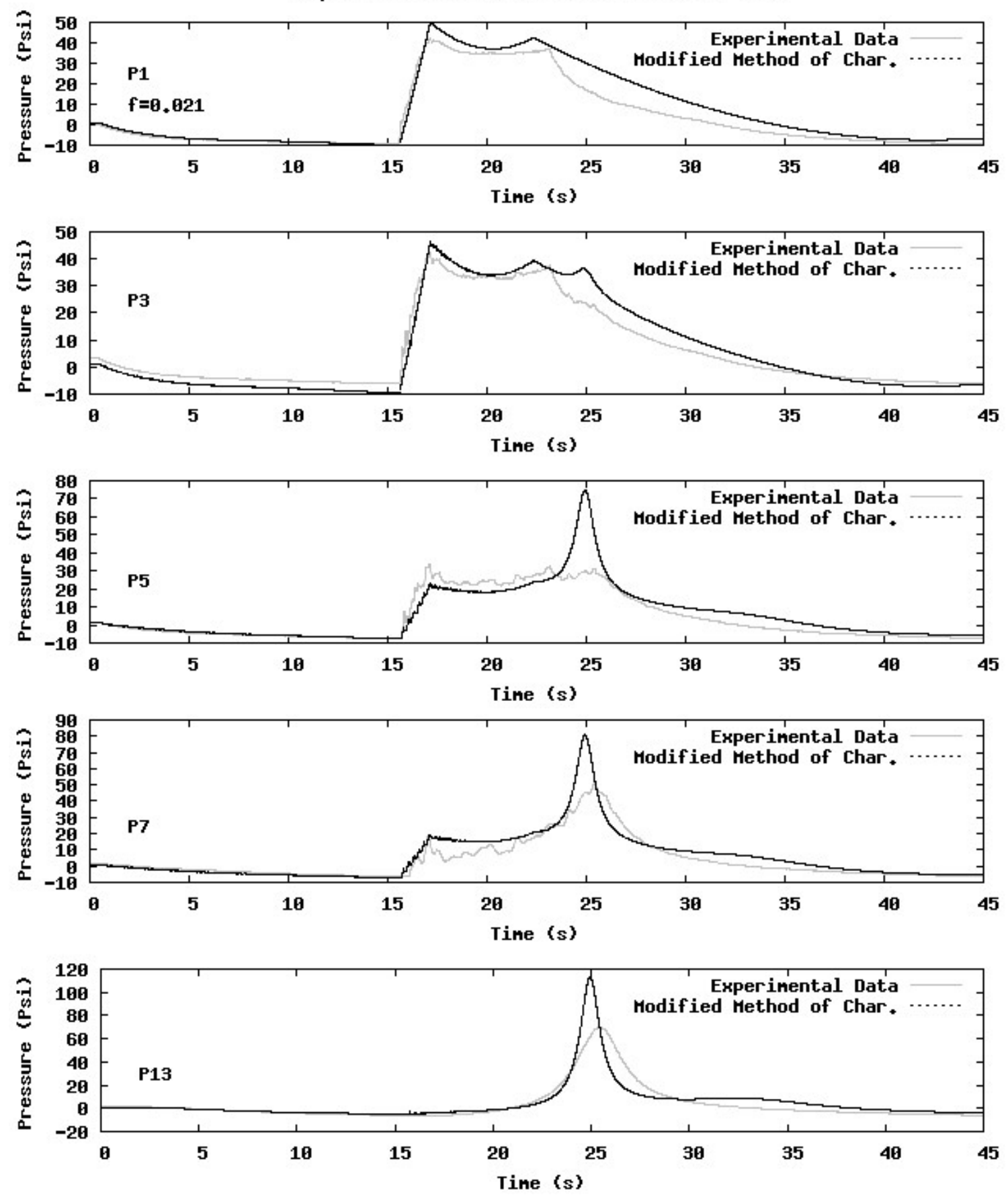

Figure 5-31: Interim Pump Optimization Results for 621ft Case 

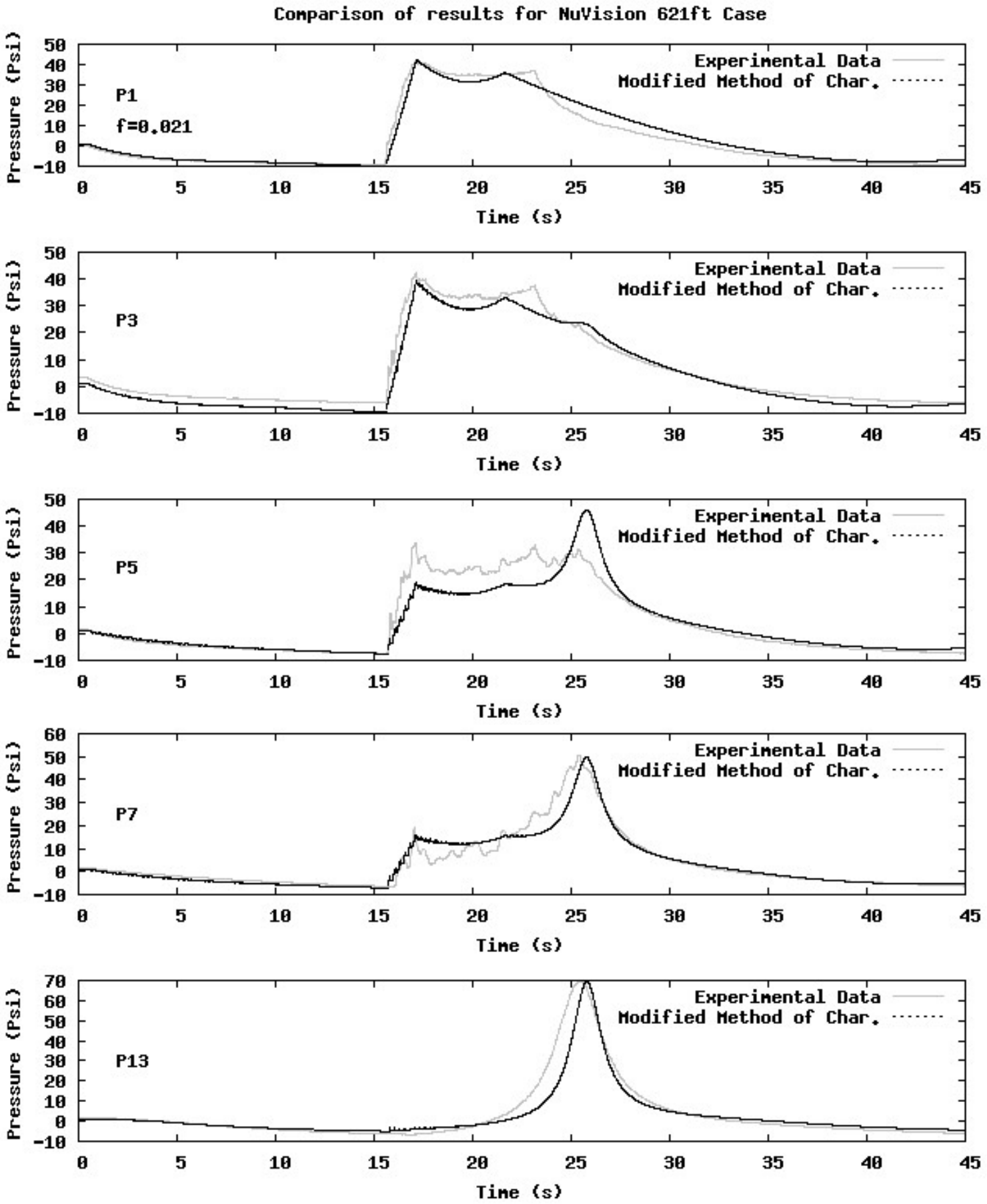

Figure 5-32: Final Pump Optimization Results for 621ft Case 


\subsubsection{7ft Test Pipeline}

The objective of the following pump operation optimization for the $1797 \mathrm{ft}$ pipeline is to produce a peak pressure of 48 Psi at pressure sensor P13 at the end of pipe segment 10. Figure 5-33 and Figure 5-34 display the pressure error and time error respectively for the ranges of hold time and applied pressure detailed in Table 2.

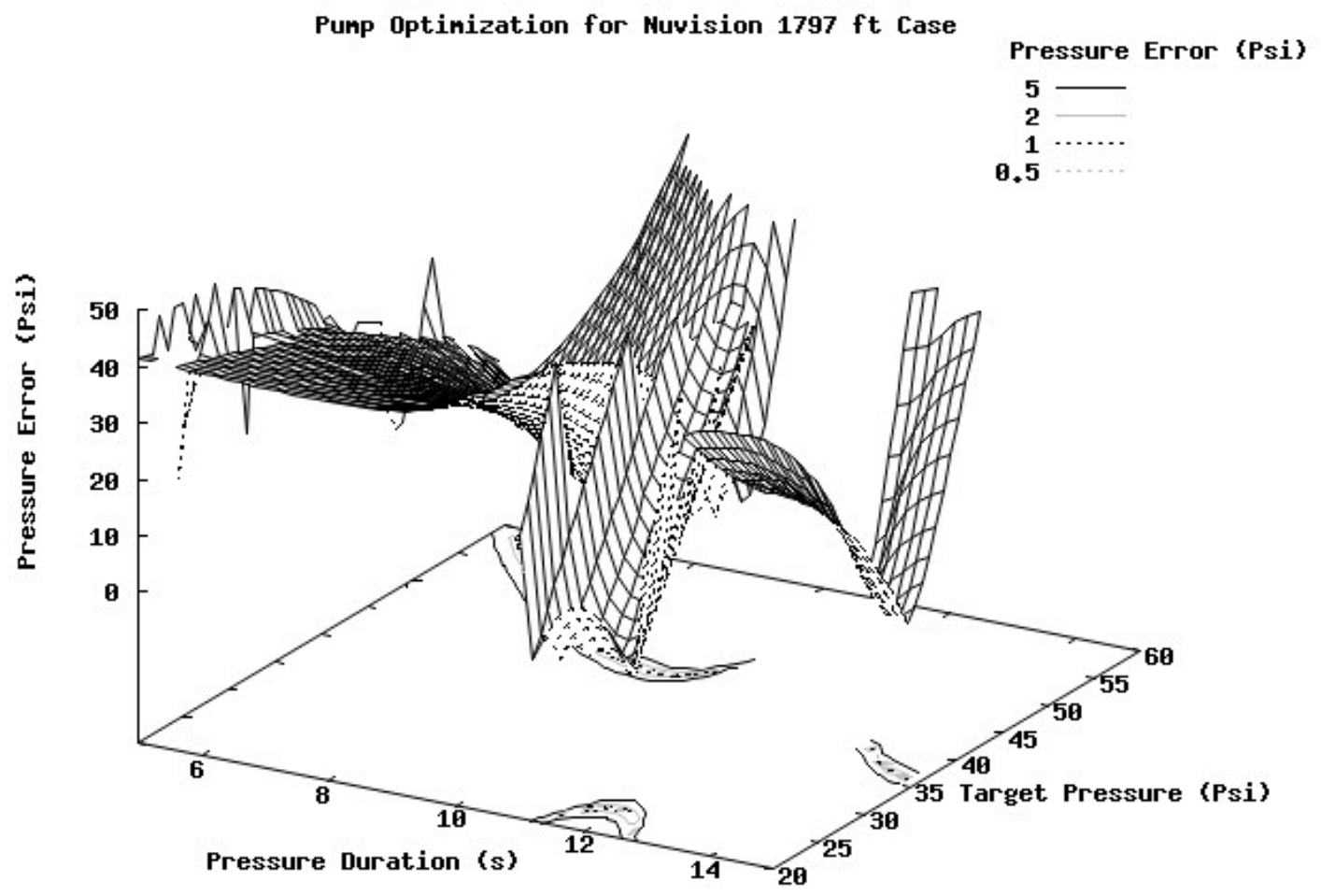

Figure 5-33: Pump Operation Optimization for NuVision 1797ft Pipeline by Pressure Error 


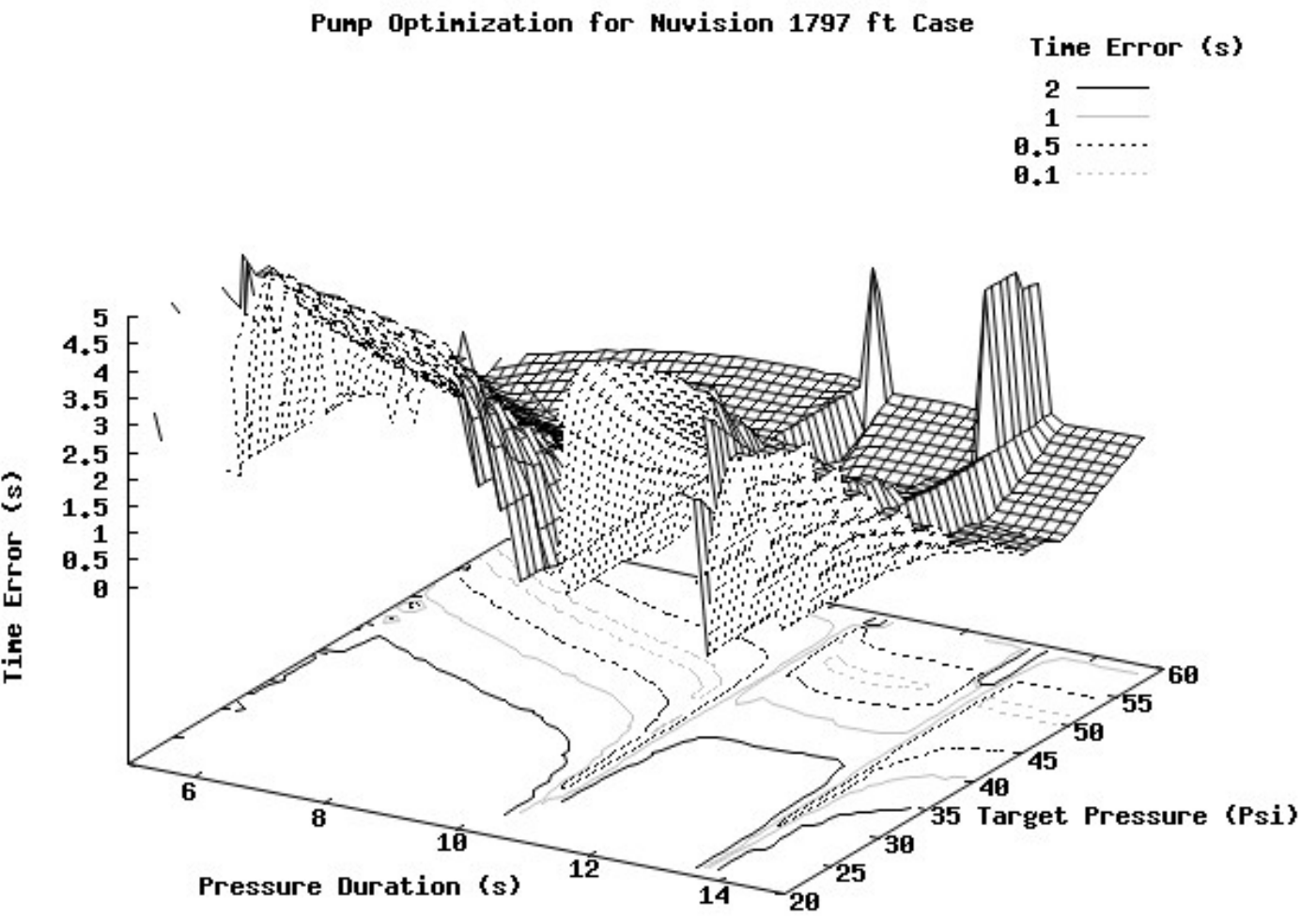

Figure 5-34: Pump Operation Optimization for NuVision 1797ft Pipeline by Time Error

Examination of Figure 5-33 and Figure 5-34 reveals that it is possible to achieve peak pressures of 73 Psi at pressure sensor P13 for applied pressures between 20 and 60 psi by maintaining the applied pressure for specific intervals for particular pressures. This test case reveals the highly non-linear behavior of transients in a long pipeline with $90^{\circ}$ elbows.

Figure 5-35, Figure 5-36 and Figure 5-37 display the pressure time histories for the initial, an intermediate and the final sets of pump optimization parameters as specified in Table 9. 
The results show that the pump operation optimization by a genetic algorithm can provide a specific recommendation for a pumping schedule to achieve a desired pressure at a particular position in the $1797 \mathrm{ft}$ pipeline. Optimization also reveals the response of the system over the range of the input parameters.

Table 9: Pump Operation Optimization Summary for $1797 \mathrm{ft}$ Case

\begin{tabular}{|l|l|l|l|l|}
\hline & $\begin{array}{c}\text { Hold Time } \\
(\mathrm{s})\end{array}$ & $\begin{array}{c}\text { Applied Pressure } \\
(\mathrm{Psi})\end{array}$ & $\begin{array}{c}\text { Pressure Error } \\
(\mathrm{Psi})\end{array}$ & $\begin{array}{c}\text { Time Error } \\
(\mathrm{s})\end{array}$ \\
\hline Initial & 7.6 & 40.0 & 19.5 & 1.9 \\
\hline Interim & 10.0 & 38.4 & 19.7 & 1.0 \\
\hline Final & 5.6 & 57.6 & 0.22 & 0.22 \\
\hline
\end{tabular}



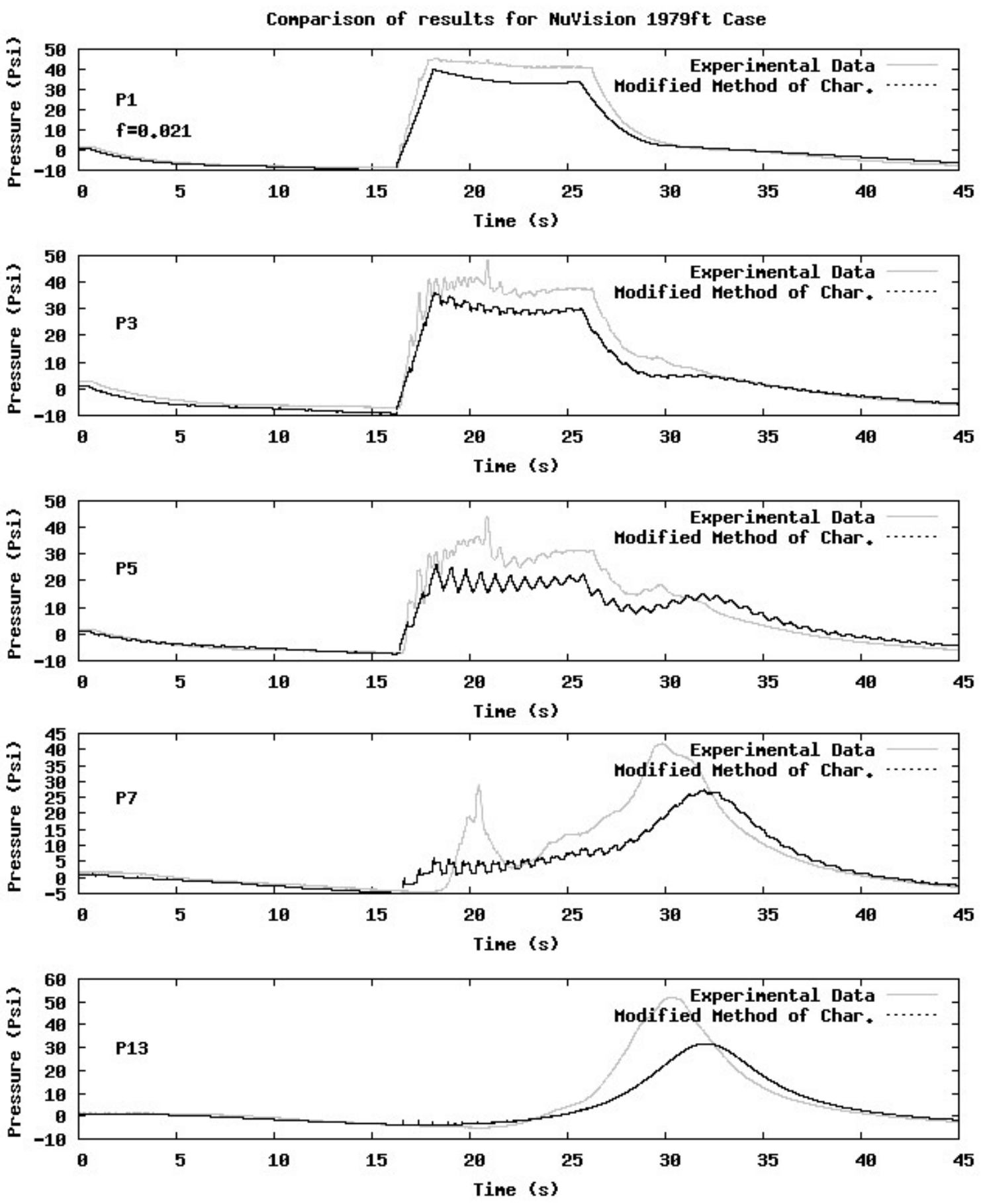

Figure 5-35: Initial Pump Optimization Results for 1797ft Case 

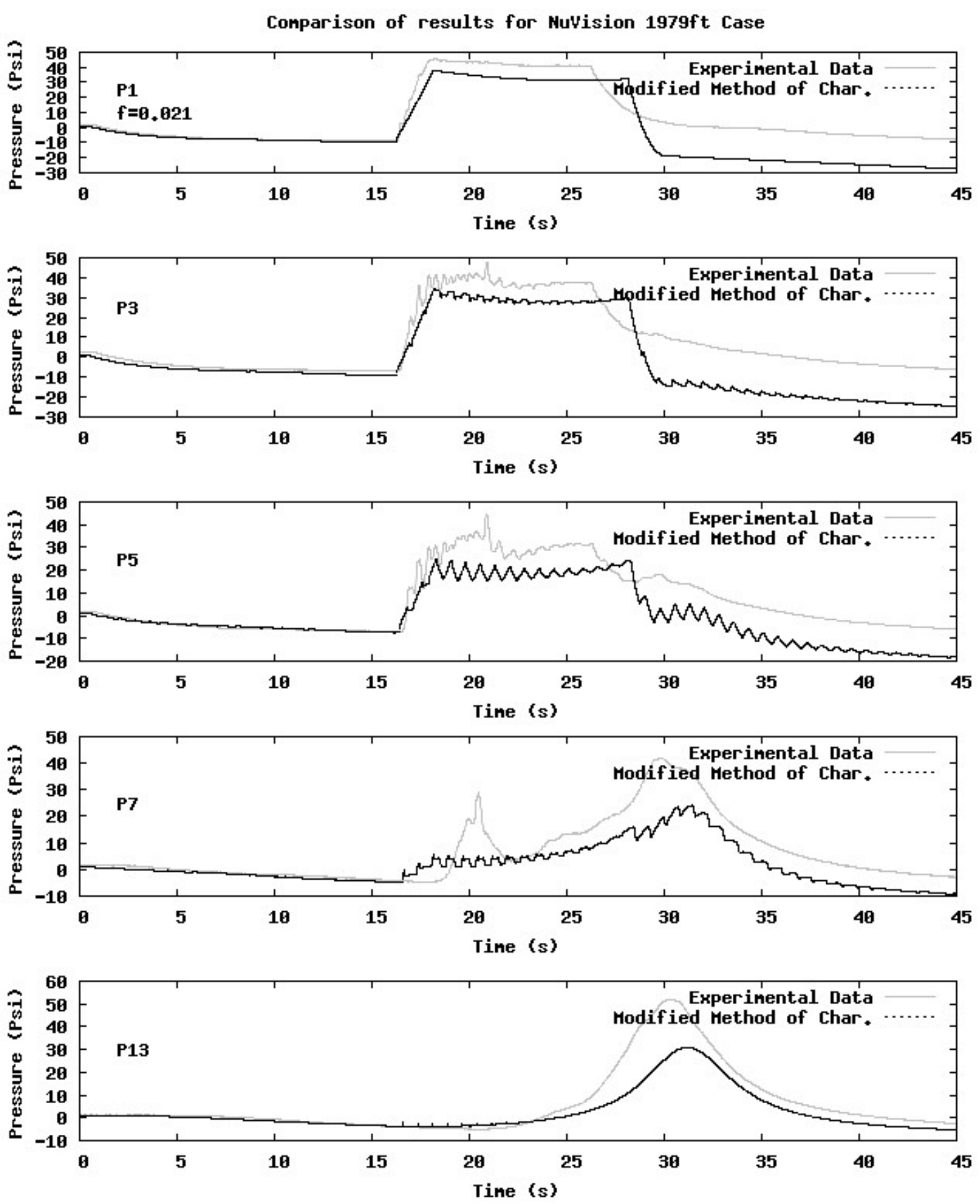

Figure 5-36: Interim Pump Optimization Results for $1797 \mathrm{ft}$ Case 

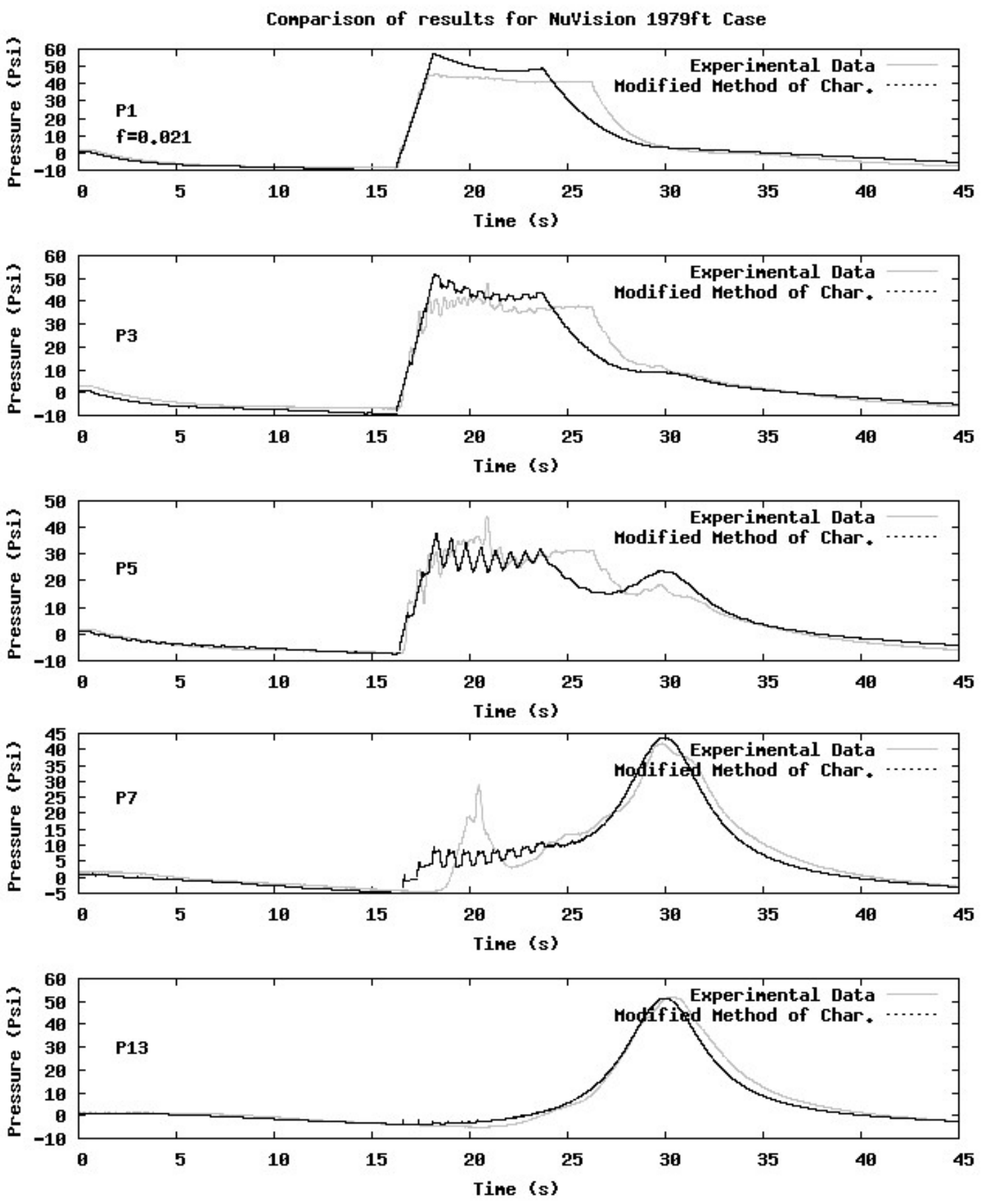

Figure 5-37: Final Pump Optimization Results for $1797 \mathrm{ft}$ Case 


\subsection{Blockage Detection}

After a pipeline has been characterized as detailed in section 4.3 the MMOC model can be utilized for blockage detection as explained in section 4.5.

\subsubsection{5ft Test Pipeline}

Initial Pipeline for Blockage Detection in MuVision $285 \mathrm{ft}$ Case

MuVision $285 f t$.......

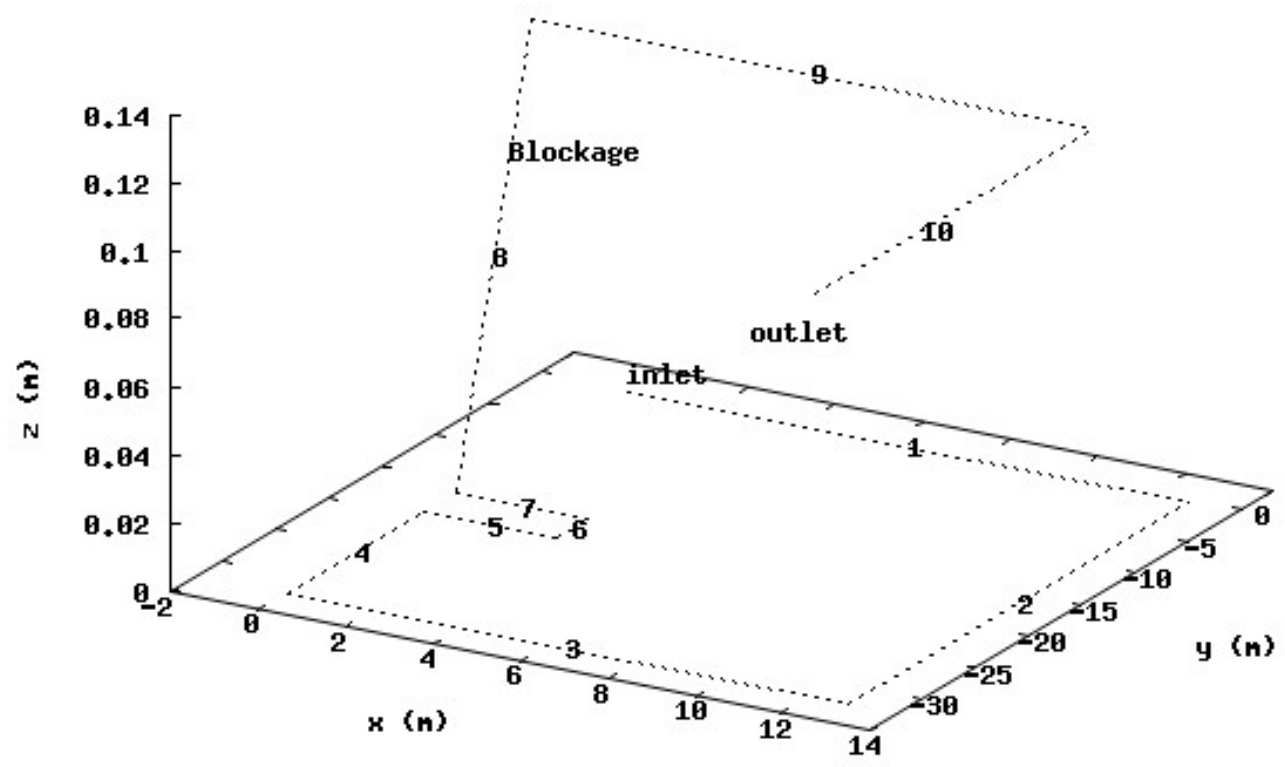

Figure 5-38: Initial Pipeline for Blockage Detection in 285ft Case

The MMOC model of the initial pipeline utilized in detecting a blockage by matching the pressure trace at the inlet is shown in Figure 5-38. The model pipeline is progressively shortened to locate the blockage as detailed in section 4.5. At each length the peak pressure and its time of occurrence is compared with the pressure time history 
from the inlet. The pressure error and time error are displayed in Figure 5-39 as the function of pipeline length.

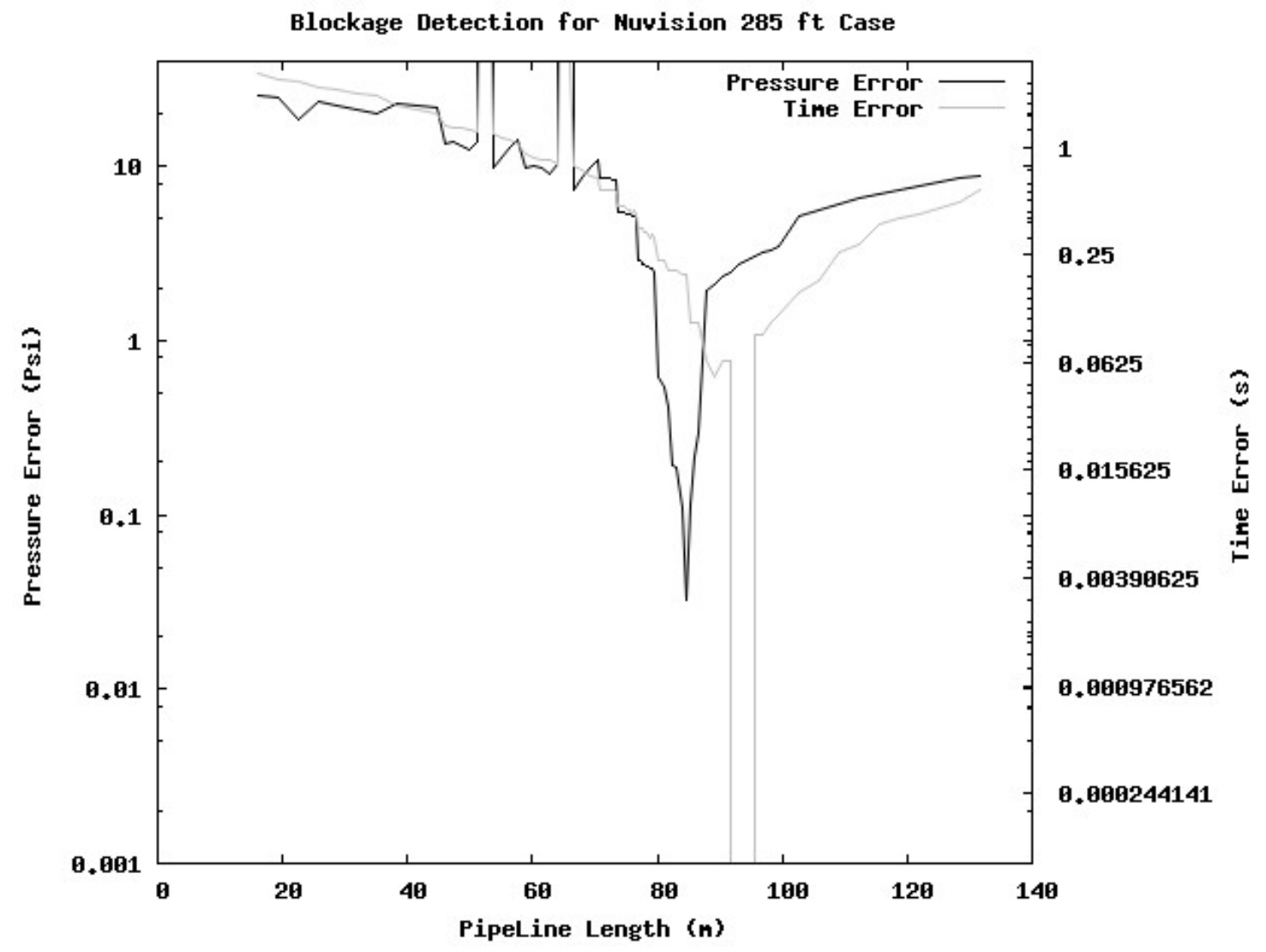

Figure 5-39: Blockage Detection Results for $285 f$ Case

The data shown in Figure 5-39 shows blockage detection by progressively shortening a MMOC pipeline model has great sensitivity for both pressure and time. The indicated minimums of pressure error and time error bracket the actual location of the blockage at $285 \mathrm{ft}(86.9 \mathrm{~m})$ between $83 \mathrm{~m}$ and $90 \mathrm{~m}$. 


\subsubsection{1ft Test Pipeline}

Initial Pipeline for Blockage Detection in MuVision 621ft Case MuVision 621ft

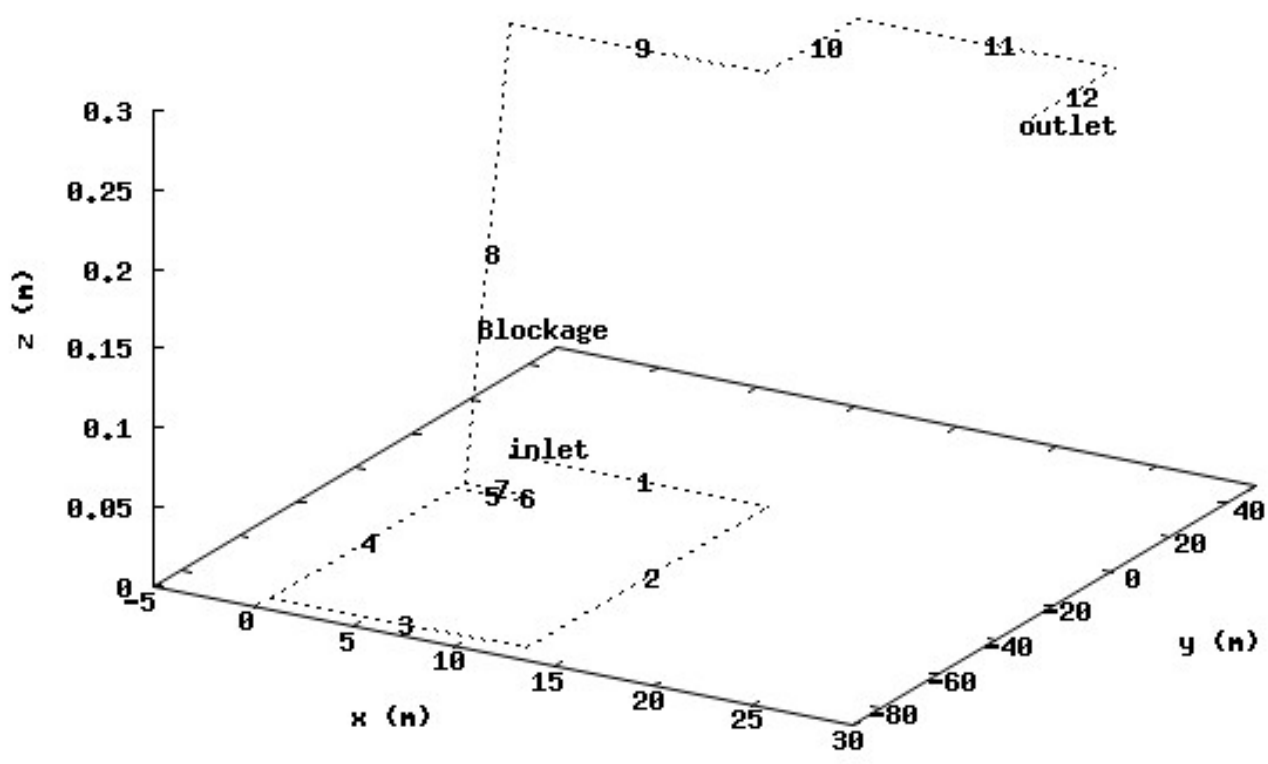

Figure 5-40: Initial Pipeline for Blockage Detection in $621 \mathrm{ft}$ Case

The MMOC model of the initial pipeline utilized in detecting a blockage by matching the pressure trace at the inlet is shown in Figure 5-40. The model pipeline is progressively shortened to locate the blockage as detailed in section 4.5. At each length the peak pressure and its time of occurrence is compared with the pressure time history from the inlet. The pressure error and time error are displayed in Figure 5-41 as the function of pipeline length. 


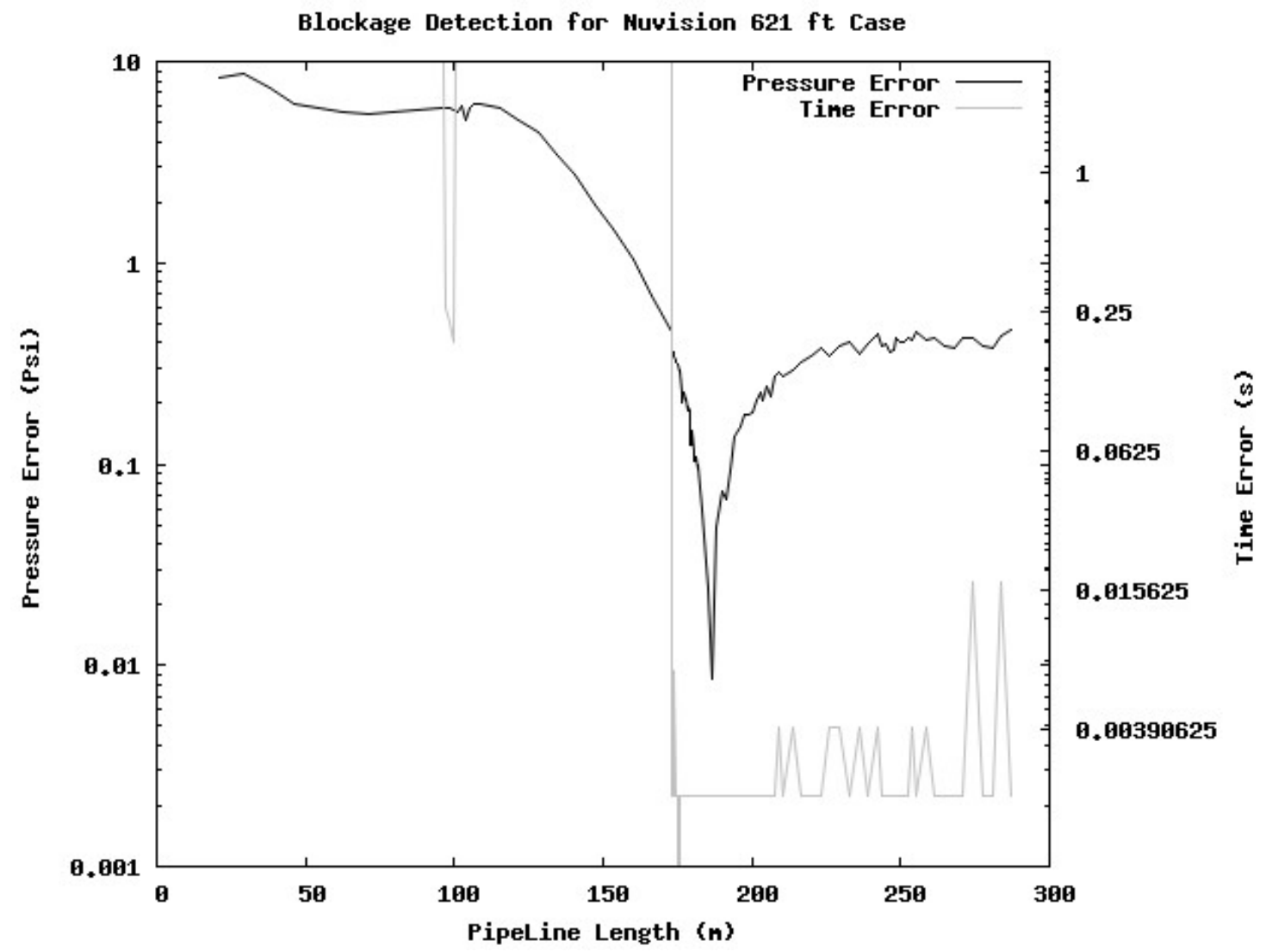

Figure 5-41: Blockage Detection Results for 621ft Case

The data shown in Figure 5-40 shows blockage detection by progressively shortening a MMOC pipeline model has great sensitivity for both pressure and time. The indicated minimums of pressure error and time error bracket the actual location of the blockage at $621 \mathrm{ft}(189.3 \mathrm{~m})$ between $180 \mathrm{~m}$ and $191 \mathrm{~m}$. 


\subsubsection{7ft Test Pipeline}

Initial Pipeline for Blockage Detection in NuVision $1797 \mathrm{ft}$ Case

MuYision $1797 \mathrm{ft}$

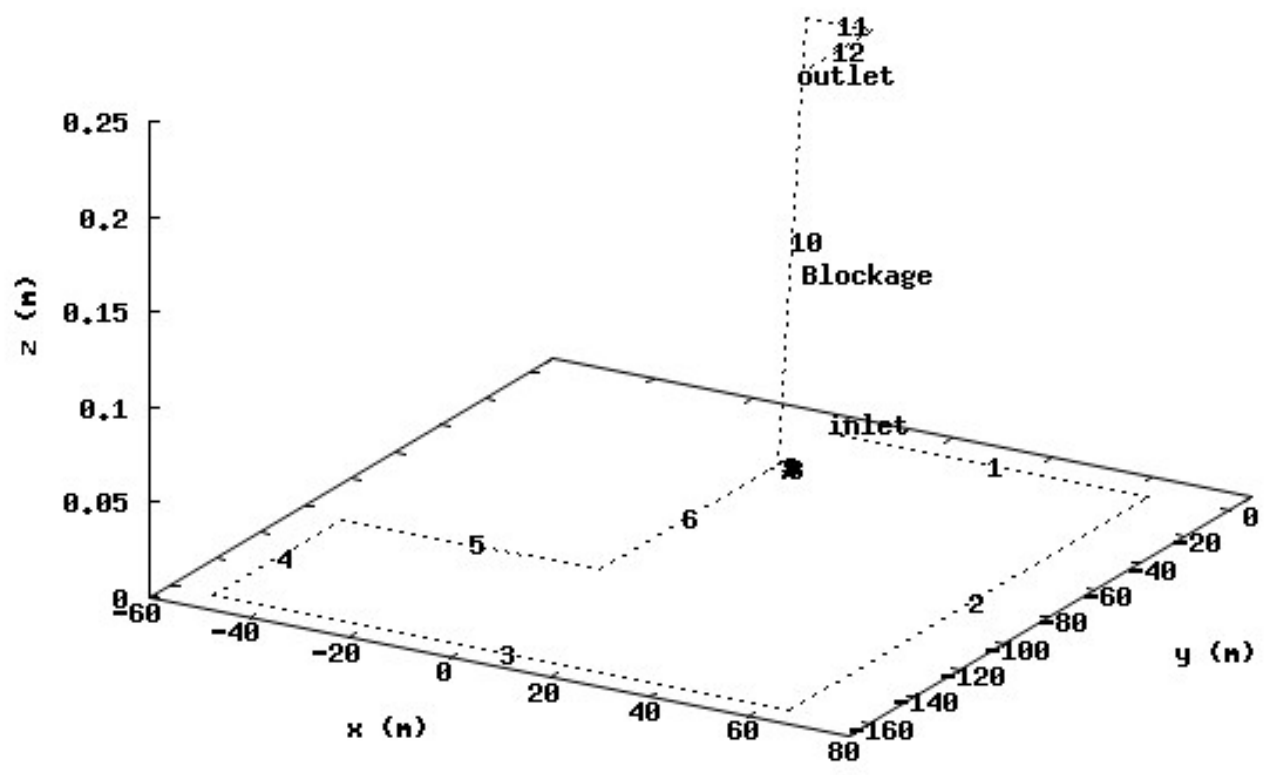

Figure 5-42: Initial Pipeline for Blockage Detection in 1797ft Case

The MMOC model of the initial pipeline utilized in detecting a blockage by matching the pressure trace at the inlet is shown in Figure 5-42. The model pipeline is progressively shortened to locate the blockage as detailed in section 4.5. At each length the peak pressure and its time of occurrence is compared with the pressure time history from the inlet. The pressure error and time error are displayed in Figure 5-43 as the function of pipeline length. 


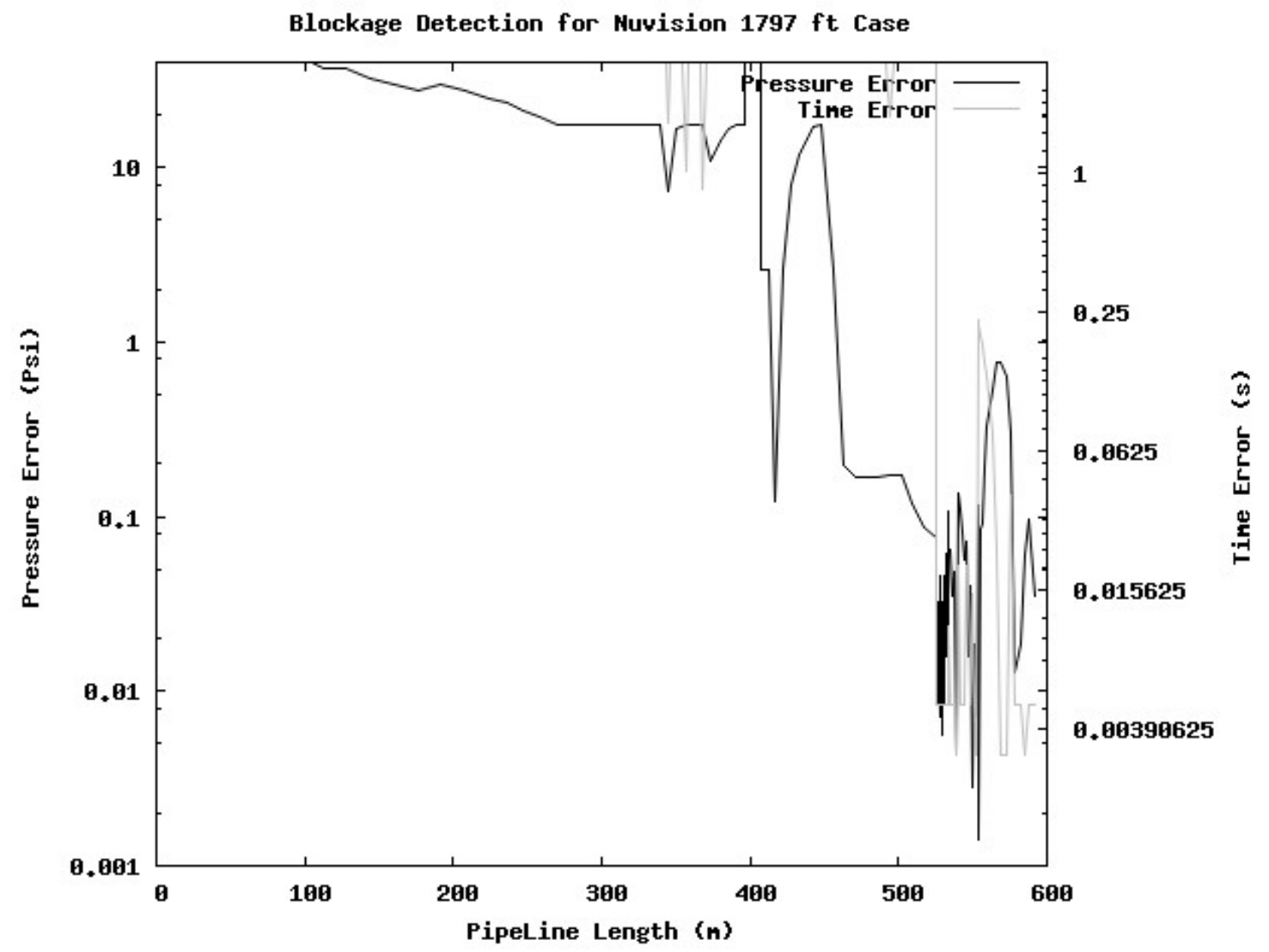

Figure 5-43: Blockage Detection Results for $1797 \mathrm{ft}$ Case

The data shown in Figure 5-43 shows blockage detection by progressively shortening a MMOC pipeline model has great sensitivity for both pressure and time. The indicated minimums of pressure error and time error bracket the actual location of the blockage at $1797 \mathrm{ft}(547.7 \mathrm{~m})$ between $530 \mathrm{~m}$ and $560 \mathrm{~m}$.

\subsection{Blockage Removal}

After a pipeline has been characterized as detailed in section 4.3 and the blockage has been detected as explained in section 4.5 the MMOC model for blockage removal can be applied as described in section 4.6. 


\subsubsection{Verification}

Figure 5-44 and Figure 5-46 show the good agreement of the axisymmetric model implemented in the MeshFree method [5, 42] on the left with the full 3D model analyzed in SolidWorks on the right. Figure 5-45 and Figure 5-47 show the radial displacement distribution and axial displacement distribution in elevation respectively. Table 10 summarizes the results of the displacement convergence study.

Table 10: Convergence of Displacement

\begin{tabular}{|l|l|l|l|}
\hline SolidWorks & meshFree \\
\hline Medium Resolution & 6,784 elements & Medium Resolution & $10,000 \mathrm{DoF}$ \\
\hline Max radial displacement & $1.79 \mathrm{e}-07 \mathrm{ml}$ & Max radial displacement & $1.79 \mathrm{e}-07 \mathrm{ll}$ \\
\hline Max axial displacement & $1.08 \mathrm{e}-06 \mathrm{~m}$ & Max axial displacement & $1.09 \mathrm{e}-06 \mathrm{~m}$ \\
\hline \multicolumn{2}{|c|}{ Agreement } \\
\hline Max radial displacement & \multicolumn{3}{|c|}{$\mathbf{0 . 4 2 4 \%}$} \\
\hline Max axial displacement & $\mathbf{0 . 0 7 4 \%}$ \\
\hline
\end{tabular}

\begin{tabular}{|c|c|c|c|}
\hline \multicolumn{2}{|l|}{ SolidWorks } & \multicolumn{2}{|l|}{ meshFree } \\
\hline F'ine Resolution & $\$ 2,229$ elements & F'ine Resolution & $80,000 \mathrm{DoH}$ \\
\hline Max radial displacement & $1.83 \mathrm{e}-07 \mathrm{~m}$ & Max radial displacement & $1.84 \mathrm{e}-07 \mathrm{~m}$ \\
\hline Max axial displacemenl & $1.09 \mathrm{e}-06 \mathrm{ml}$ & Mis axial displacement & $1.09 \mathrm{e}-06 \mathrm{mll}$ \\
\hline \multicolumn{4}{|c|}{ Agreement } \\
\hline \multicolumn{2}{|l|}{ Max radial displacement } & \multicolumn{2}{|l|}{$0.346 \%$} \\
\hline \multicolumn{2}{|l|}{ Max axial displacement } & \multicolumn{2}{|l|}{$0.054 \%$} \\
\hline
\end{tabular}



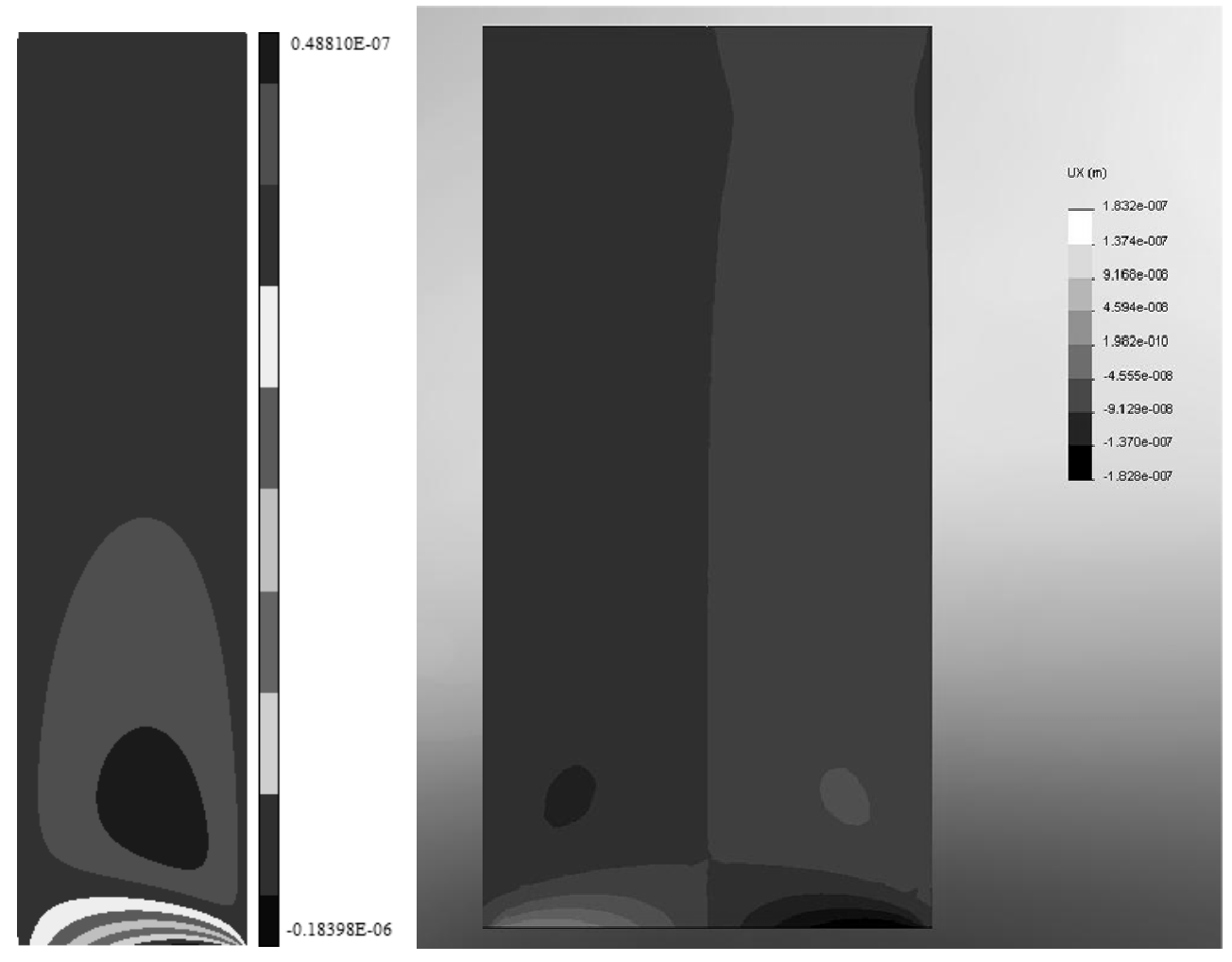

Figure 5-44: Verification of Radial Displacement Distribution 


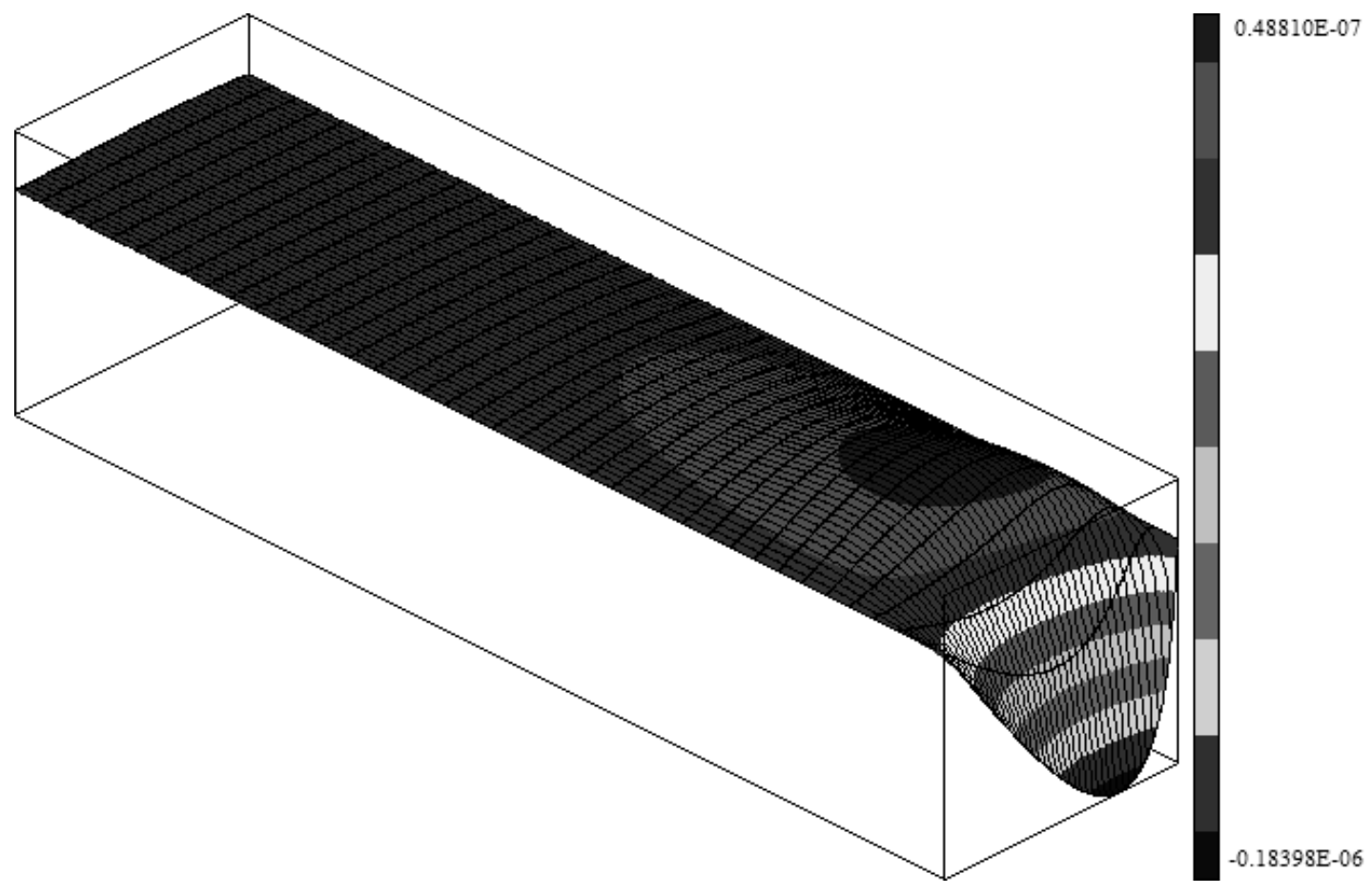

Figure 5-45: Verification of Radial Displacement Distribution shown in elevation
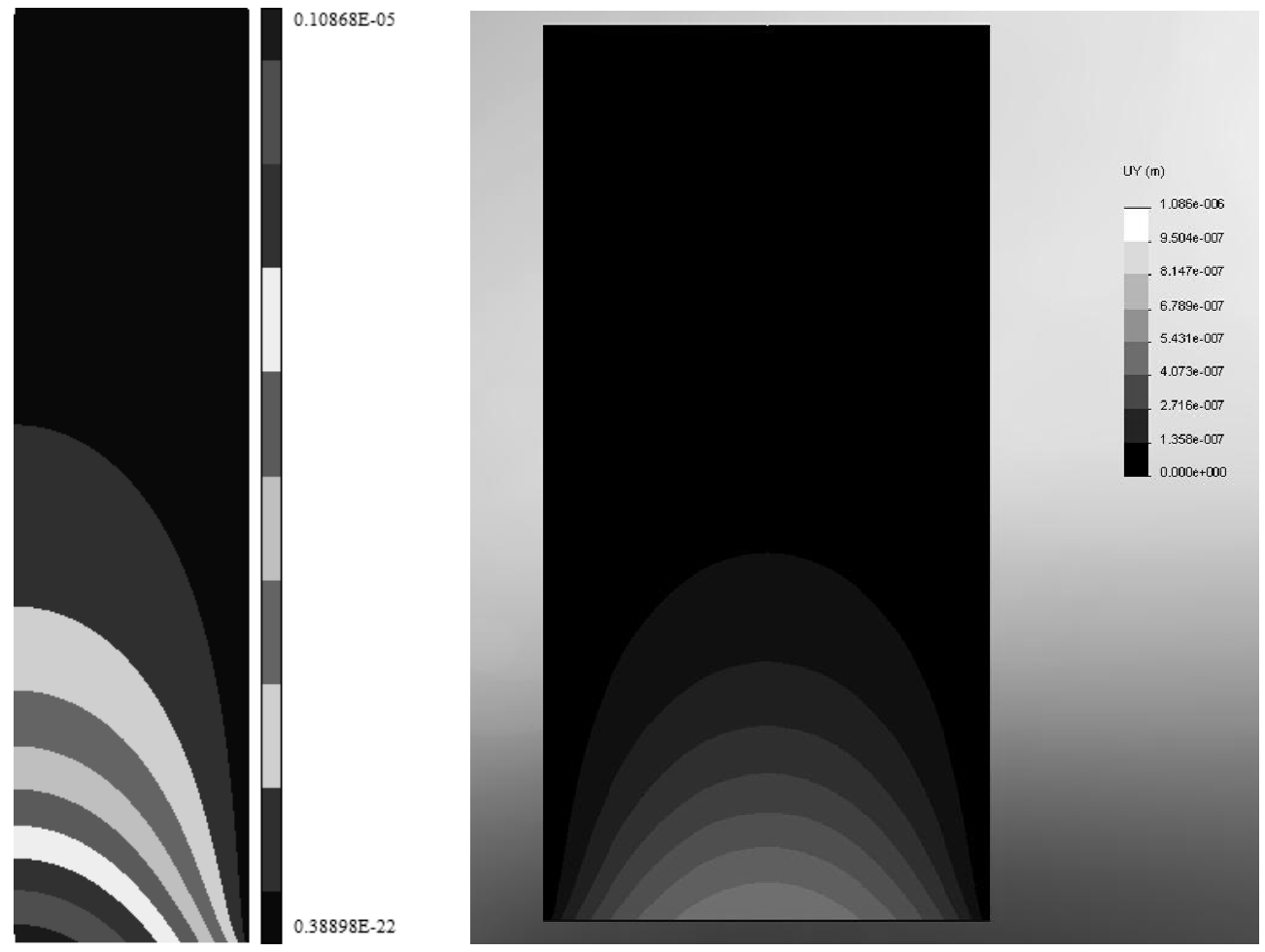

Figure 5-46: Verification of Axial Displacement Distribution 


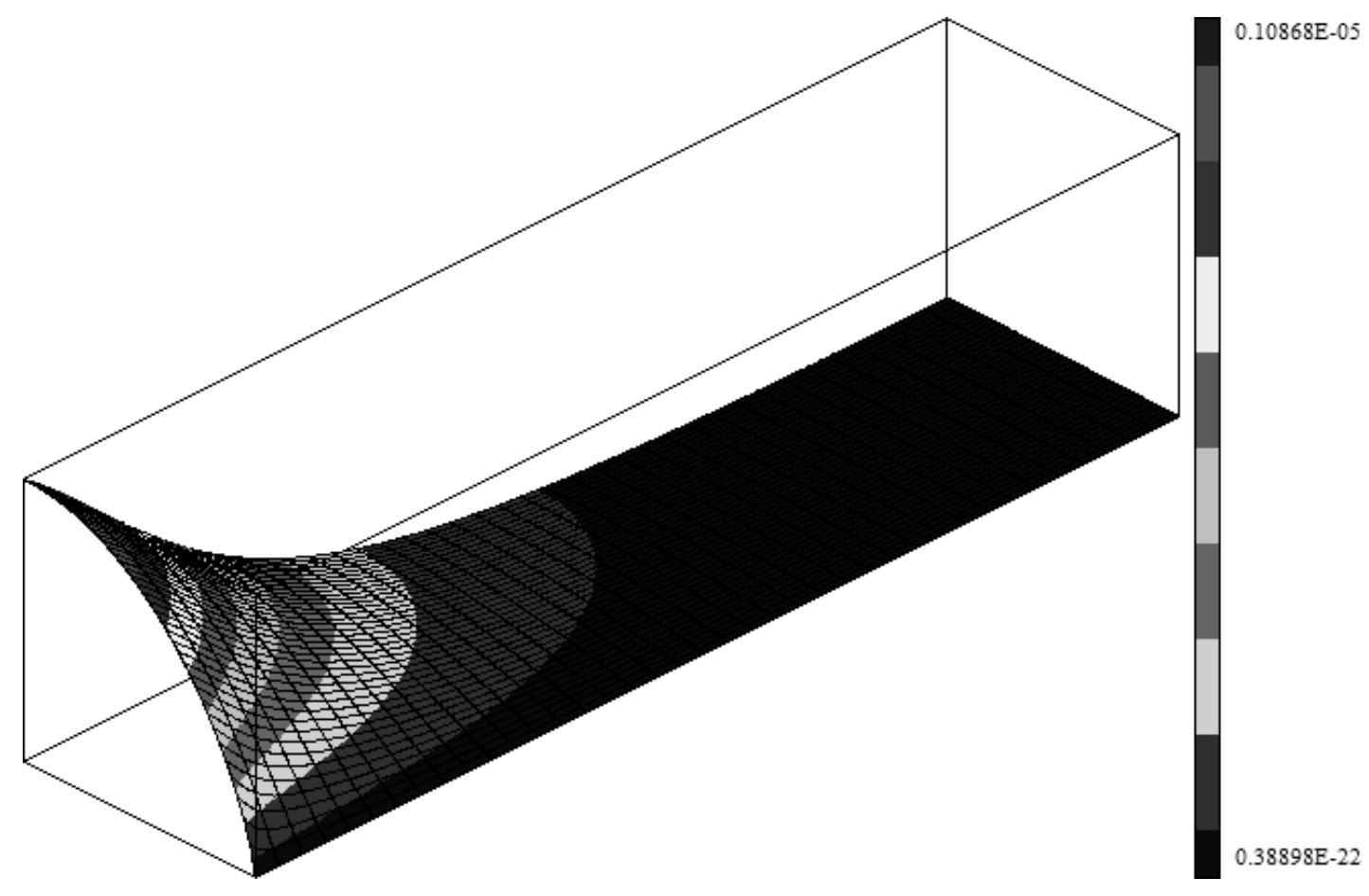

Figure 5-47: Verification of Axial Displacement Distribution shown in elevation

Table 11 presents the relative percentages of improvement gained by increasing the refinement of the solid works and meshFree simulations by a factor of eight between the medium and fine resolution studies. This barely perceptible change indicates that the medium resolution meshFree study adequately resolves the deformation of the blockage as modeled in this work. This resolution is used for integration with the MMOC.

Table 11: Relative Improvement of Displacement Study

\begin{tabular}{|l|l|l|}
\hline Improvement (medium to fine) & SolidWorks & meshFree \\
\hline Max radial displacement & $0.002 \%$ & $0.003 \%$ \\
\hline Max axial displacement & $0.009 \%$ & $0.001 \%$ \\
\hline
\end{tabular}




\subsubsection{Integration with MMOC}

The pipeline model used in MMOC for the $285 \mathrm{ft}$ blockage removal case is shown in Figure 5-48. The blockage is $4 \mathrm{ft}$ in length and completely fills the 3 inch diameter pipe and is located at the end of pipe 8. Pipes 9 and 10 extend for a total of 20 meters beyond the blockage. The parameters utilized in this case are listed in Table 12.

Table 12: Parameters for 285ft Blockage Removal Case

\begin{tabular}{|l|c|}
\hline Blockage length & $1.22 \mathrm{~m}$ \\
\hline Blockage mass & $5 \mathrm{~kg}$ \\
\hline Blockage static friction factor & 0.5 \\
\hline Blockage dynamic friction factor & 0.3 \\
\hline Length to pipeline outlet & $20 \mathrm{~m}$ \\
\hline
\end{tabular}


Pipeline for MuVision 285ft Blockage Renoval Case

MuVision 285ft

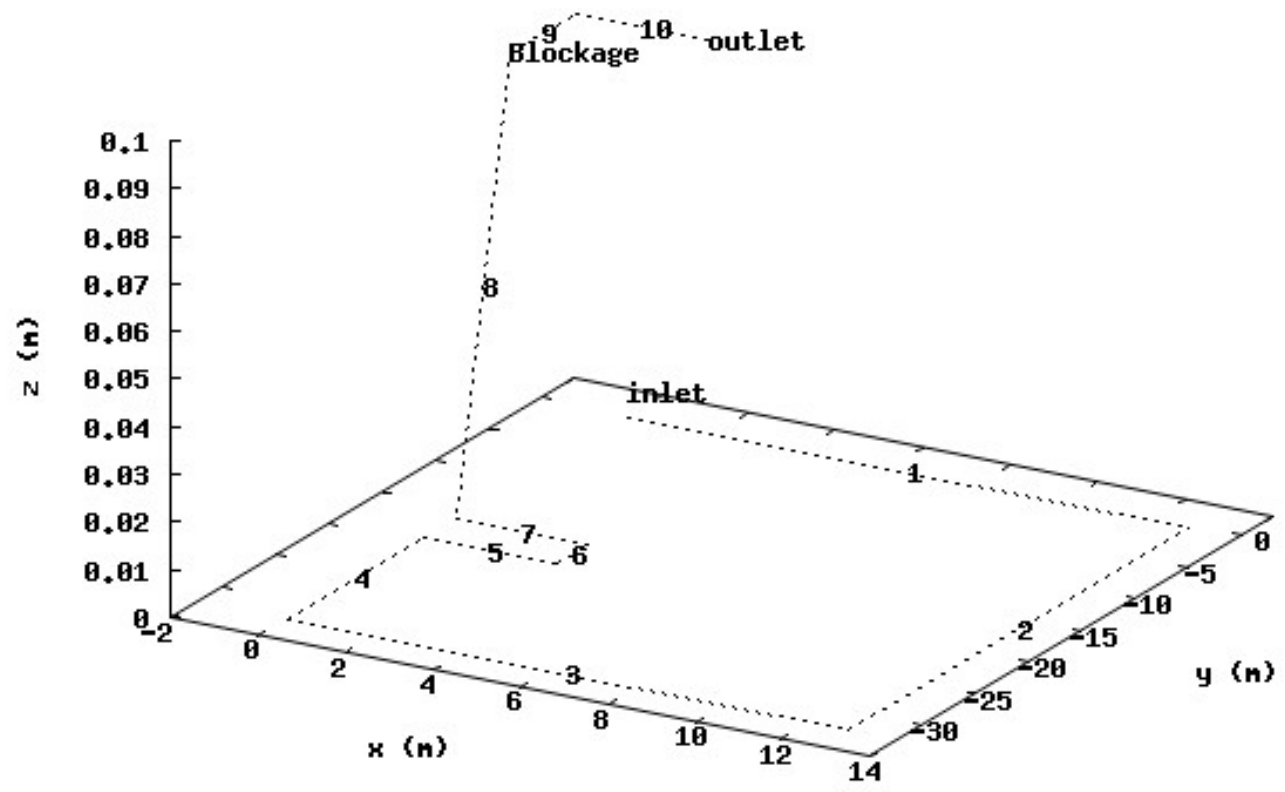

Figure 5-48: MMOC Pipeline Model for Blockage Removal for $285 \mathrm{ft}$ Case

Figure 5-49 shows the pressures predicted by MMOC at pressure sensors P1, P3, $\mathrm{P} 5, \mathrm{P} 7$, and P13. It can be noted from these pressure histories that the blockage is predicted to exit the pipeline at approximately 16 seconds. This correlates with the predicted plug motion show in Figure 5-50. 
Blockage Renoval for MuVision 285ft Case
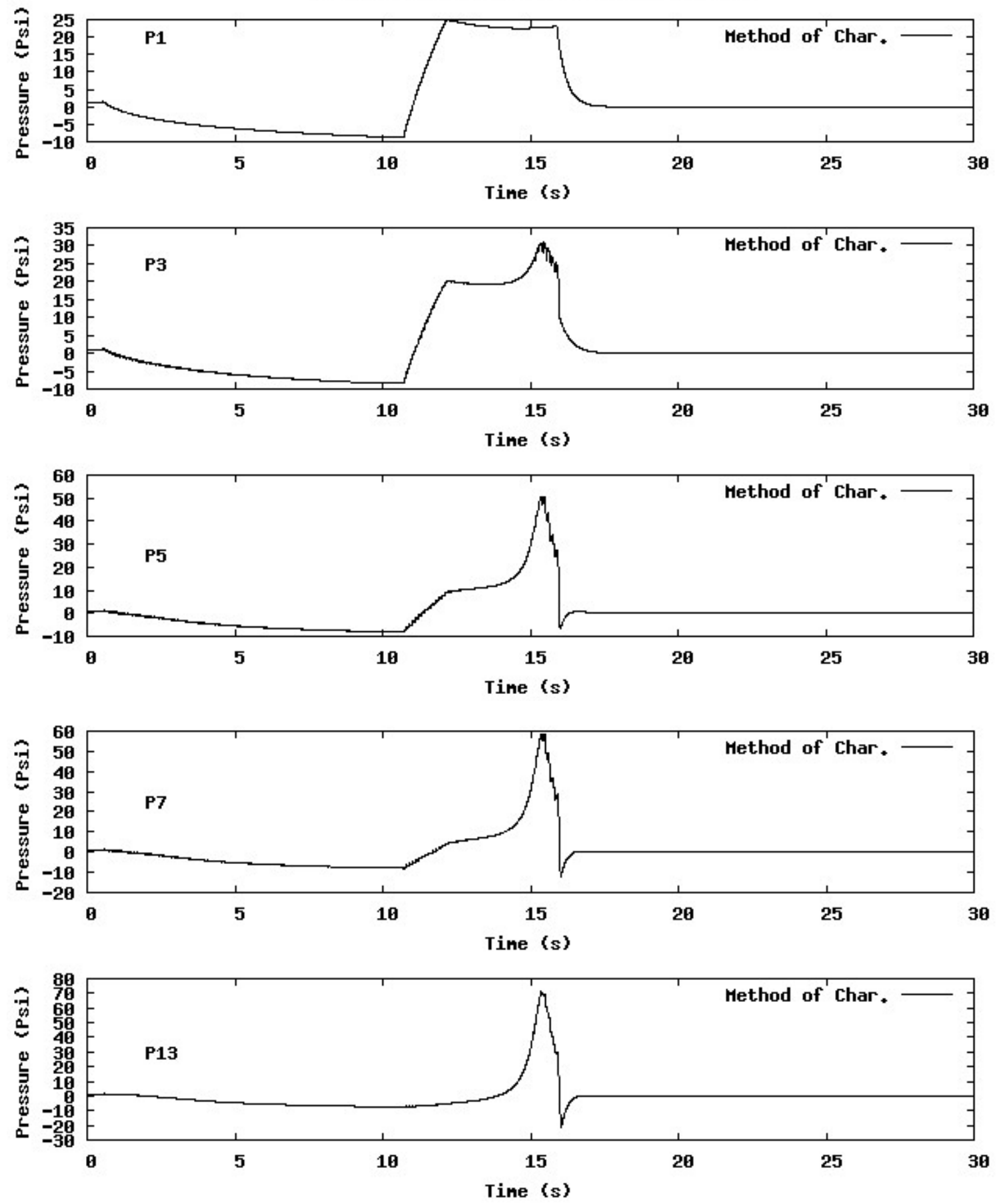

Figure 5-49: Pressure History for Blockage Removal in $285 \mathrm{ft}$ Case 


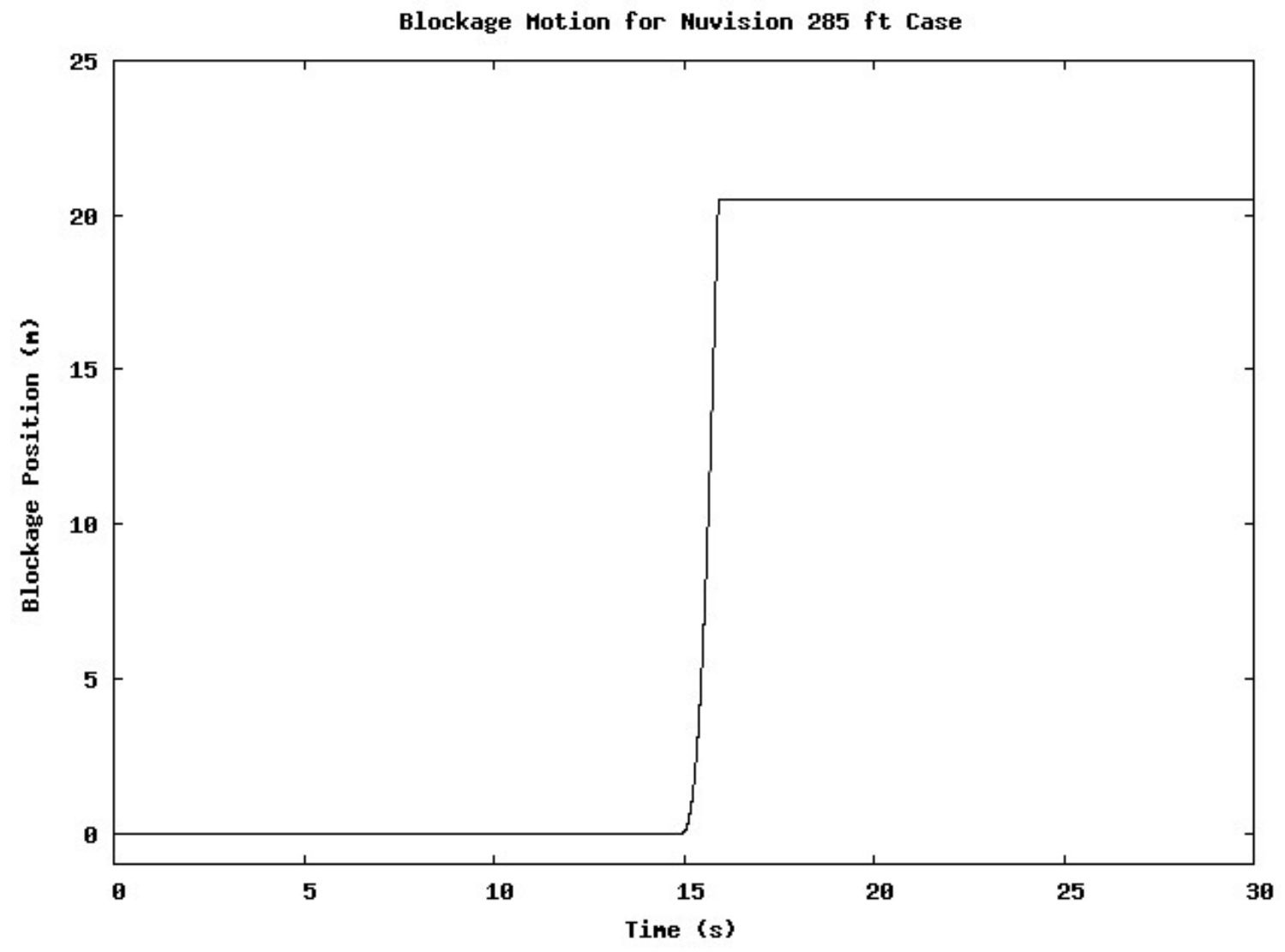

Figure 5-50: Blockage Motion for 285ft Case 


\section{CHAPTER VI}

\section{CONCLUSIONS AND FUTURE WORK}

Pipeline characterization by optimization with a differential evolution genetic algorithm has been shown to produce accurate and predictive MMOC models. The result of this characterizing optimization is the friction factor, $f$, and loss coefficient, $c_{g}$, that minimize the difference between the peak pressure predicted by MMOC and the experimental data as well as the error between the predicted time of occurrence of the peak pressure and the experimental data.

The web application created for MMOC has shown that PHP can support a secure, user friendly and error checking interface. The web application enables timely model creation, simulation, and results visualization of pipeline characterization.

After the friction factor, $f$, and loss coefficient, $c_{g}$, have been determined for a pipeline the same MMOC model and differential evolution genetic algorithm can be successfully used to efficiently determine the optimum pump operation schedule to achieve a desired pressure at a particular position in a pipeline.

A complete blockage in a pipeline that has been characterized can be located by the novel approach of progressively shortening the MMOC model of the pipeline until the predicted peak pressure at the inlet matches the experimental data at the inlet. In the

present implementation the position of the blockage can be determined to within $10 \%$ of the length of the pipe segment where it is located. 
The integration of a solid mechanics model for the blockage with the MMOC has been verified. The predicted pressures in the $285 \mathrm{ft}$ pipeline during blockage removal and the sudden plug motion predicted correlate well with the observations from the technology evaluation.

Future work on pipeline characterization should include further experimental work to further explore the geometric loss coefficients relationship to the overall pipeline geometry. An interesting continuation to the work on pipeline characterization has been suggested by Professor Dulikravich. The Darcy-Weisbach friction factor, $f$, is a function of the pipeline's local inner surface relative roughness, $\varepsilon_{i} / D_{i}$, and the local Reynolds Number, $R_{e_{i}}=\frac{\rho a_{i}(t) D_{i}}{\mu_{i}}$. The Reynolds Number in a pipeline during a transient will vary due to the pipe segment diameter, $D_{i}$, and the local wave speed, $a_{i}(t)$, which is time dependent. In some applications, such as those found in off shore oil drilling, the temperature variance along a pipeline may not be negligible and may cause a variation in viscosity, $\mu_{i}$. Considering the variance of the Reynolds Number along the pipeline and with time and the changes in the relative roughness of the inner surface of the pipeline may increase the accuracy of the transients predicted.

The work on blockage detection should be continued through refinement of the search method that utilizes the MMOC model. Changing the length of the model pipeline until the simulated pressure at its inlet matches that of the experimental data has proven effective. Two methods of searching for the blockage location by changing the pipeline length are suggested. The first is to determine the error between MMOC simulations and 
the experimental data for a limited number of prospective blockage locations. A cubic spline should

Additionally blockage removal should be further explored experimentally and computationally to validate the model and the coupling between MMOC and a solid solver. Lastly extending this work to pipeline networks with branches and parallel pipes will broaden its applicability from transfer lines to processing systems and networks. 


\section{REFERENCES}

[1] Wylie, E. B. \& Streeter, V. L. (1978). Fluid Transients. New York, New York: McGraw-Hill Inc.

[2] Liu, H. (2003). Pipeline Engineering. Boca Raton, Florida: CRC Press LLC.

[3] Roelant, D., McDaniel, D., Gokaltun, S., Varona, J., \& Patel, R. (2008). Unplugging of High Level Waste Transfer Pipelines: NuVision Fluidic Wave-Assessment. U.S. Department of Energy Office of Environmental Management Office of Science and Technology.

[4] Tijsseling, A. (1996). Fluid-Structure Interaction In Liquid-Filled Pipe Systems: A Review (Vol. 10). Academic Press Limited.

[5] Tsukanov, I., \& Shapiro, V. (2002). The Architecture of SAGE - A Meshfree System Based On RFM. Computers , 18.

[6] Stecki, J., \& Davis, D. C. (1986). Fluid Transmission Lines - Distributed Parameter Models. ARCHIVE: Proceedings of the Institution of Mechanical Engineers, Part A: Power and Process Engineering 1983-1988, 200 (A4), 215-228.

[7] Suo, L., \& Wylie, E. B. (1990). Complex Wavespeed and Hydraulic Transients in Viscoelastic Pipes.

[8] Shepherd, J. E., \& Inaba, K. (2009). Dynamic Loading of Fluid-Filled Tubes. (A. Shukla, G. Ravichandran, \& Y. Rajapakse, Eds.)

[9] Li, Q., Yang, K., \& Zhang, L. (2003). Analytical Solution for Fluid-Structure Interaction in Liquid-Filled Pipes Subjected to Impact-Induced Water Hammer (Vol. 10). ASCE.

[10] Amein, M., \& Chu, H. L. (1975). Implicit Numerical Modeling of Unsteady Flows. Journal of the Hydraulics Division, 101 (HY6), 717-731.

[11] Wood, D. J., \& Chao, S. P. (1971). Effect of pipeline junctions on waterhammer surges. Transportation Engineering Journal, 97, 441-456.

[12] Azzi, A., Friedel, L., Kibboua, R., \& Shannak, B. (2002). Reproductive Accuracy of Two-Phase Flow Pressure Loss Corrections for vertical 90' Bends.

[13] Jones, S. E., \& Wood, D. J. (1972). The effect of axial boundary motion on pressure surge generation. Journal of Basic Engineering , 94, 441-446. 
[14] Valentin, R. A., Philips, J. W., \& Walker, J. S. (1979). Reflections and Transmission of Fluid Transients at an Elbow.

[15] Dzodzo, M. B., Liu, B., Cincolini, A., \& Spiegleman, S. R. (2006). Applciation of CFD for Modeling Flows in Feed-Water Pipelines. Proceedings of ICONE 14. Miami, FL: ICONE.

[16] Supra-Amornkul, Steward, \& Lister. (2005). Modeling Two-Phase Flow in Pipe Bends. Journal of Pressure Vessel Technology, 127.

[17] Douglas, J. F., Gasiorek, J. M., Swaffield, J. A., \& Jack, L. B. (2005). Fluid Mechanics (5th ed.). Prentice Hall Inc.

[18] Adamkowski, A., \& Lewandowski, M. (2006). Experimental Examination of Unsteady Friction Models for Transient Pipe Flow Simulation (Vol. 128). ASME

[19] Bergant, A., \& Tijsseling, A. (2001). Parameters Affecting Water Hammer Wave ttenuation, Shape and Timing. Trodheim, Norway: AIRH.

[20] Ghidaoui, M. S., Zhao, M., McInnis, D. A., \& Axworthy, D. H. (2005). A Review of Water Hammer Theory and Practice. Applied Mechanics Review , 58, 49-76.

[21] Tullis, J. P. (1989). Hydraulics of pipelines: pumps, valves, cavitation, transients. John Wilye \& Sons, Inc.

[22] Streeter, V. L., \& Wylie, E. B. (1966). Hydraulic Transients Caused by Reciprocating Pumps. ASME.

[23] Thorley, A. R. (1991). Fluid Transients in Pipeline Systems. D. \& L. George LTD.

[24] Vitkovsky, J. P., Simpson, A. R., \& Lambert, M. F. (2000, July/August). Leak Detection and Calibration Using Transients and Genetic Algorithms. Journal of Water Resource Planning and Management.

[25] Zyl, J. E., Savic, D. A., \& Walters, G. A. (2004). Operational Optimization of Water Distribution Systems Using a Hybrid Genetic Algorithm. Journal od Water Resource Planing and Management, 130 (160).

[26] Simpson, A. R., Dandy, G. C., \& Murphy, L. J. (1994). Genetic Algorithms Compared to Other Techniques for Pipe Optimization. Journal of Water Resource Planning and Management, 120 (423). 
[27] Vitkovsky, J. P., Liggett, J. A., Simpson, A. R., \& Lambert, M. F. (2003). Optimal Measurement Site Locations for Inverse Transient Analysis in Pipe Networks. Journal of Water Resoure Planning and Management, 129 (480).

[28] Wang, X.-J., Lambert, M. F., \& Simpson, A. R. (2005, May/June). Detection and Location of a Partial Blockage in a Pipeline Using Damping of Fluid Transients. Journal of Water Resources Planning and Management.

[29] Zollinger, W. T., \& Carney, F. (2004). Pipeline Blockage Unplugging and Locating Equipment. ANS 10th International Topical Meeting on Robotics and Remote Systems for Hazardous Environments. Idaho National Engieering and Environmental Laboratory.

[30] Mohaparta, P., Chaudhry, M. H., Kassem, A. A., \& Moloo, J. (2006, February). Detection of Partial Blockages in Single Pipelines. Journal of Hydraulic Engineering.

[31] Pitchford, J. (2002). Blockage Location - The Pulse Method. Pigging Porducts and Services Association.

[32] Chaudhry, M. H. (1979). Applied Hydraulic Transients. Litton Educational Publishing Inc. 1979.

[33] Bieber, M. (1998). Web Engineering. New Jersy Center for Multimedia Research.

[34] Ookla. (n.d.). Net Index. Retrieved June 16, 2011, from Net Index:

www.netindex.com/download/2,1/United-States/

[35] Izqueiro, J., \& Iglesias, P. L. (2002). Mathematical Modeling of Hydraulic Transients in Simple Systems.

[36] Izqueiro, J., \& Iglesias, P. L. (2004). Mathematical Modelling of Hydraulic Transients in Simple Systems.

[37] Papanastasiou, T. C., Georgiou, G. C., \& Alexandrou, A. N. (2000). Viscous Fluid Flow. CRC Press LLC.

[38] Brunone, B., Karney, B. W., Mecharelli, M., \& Ferrante, M. (2000). Velocity

Profiles and Unsteady Pipe Friction in Transient Flow.

[39] Dulikravich, G. S., \& Chowdury, S. (2010). Improvements to Singe-Objective Constrained Predator-Prey Evolutionary Optimization Algorith (Vol. 41).

[40] Krysl, P. (2006). A Pragmatic Introduction to the Finite Element Method for Thermal and Stress Analysis. World Scientific Publishing Co. Pte. Ltd. 
[41] Washizu, K. (1974). Variational Methods in Elasticity \& Plasticity (3rd ed.). Tokyo, Japan: Pergamon Press.

[42] Shapiro, V., \& Tsukanov, I. G. (2004). Patent No. 6718291 B1. US.

[43] Wood, S. (2008, January). MAIDROC Laboratory. Retrieved May 2011, from Multidisciplinary Analysis, Inverse Design, Robust Optimization and Control Laboratory: www.maidroc.fiu.edu

[44] GNU. (2011, March). GNUplot. (GNU) Retrieved May 2011, from GNUplot: http://www.gnuplot.info/ 


\section{APPENDIX}

\section{A: CODE SNIPPET}

\section{A.1 Airpocket at Downstream End Boundary Condition}

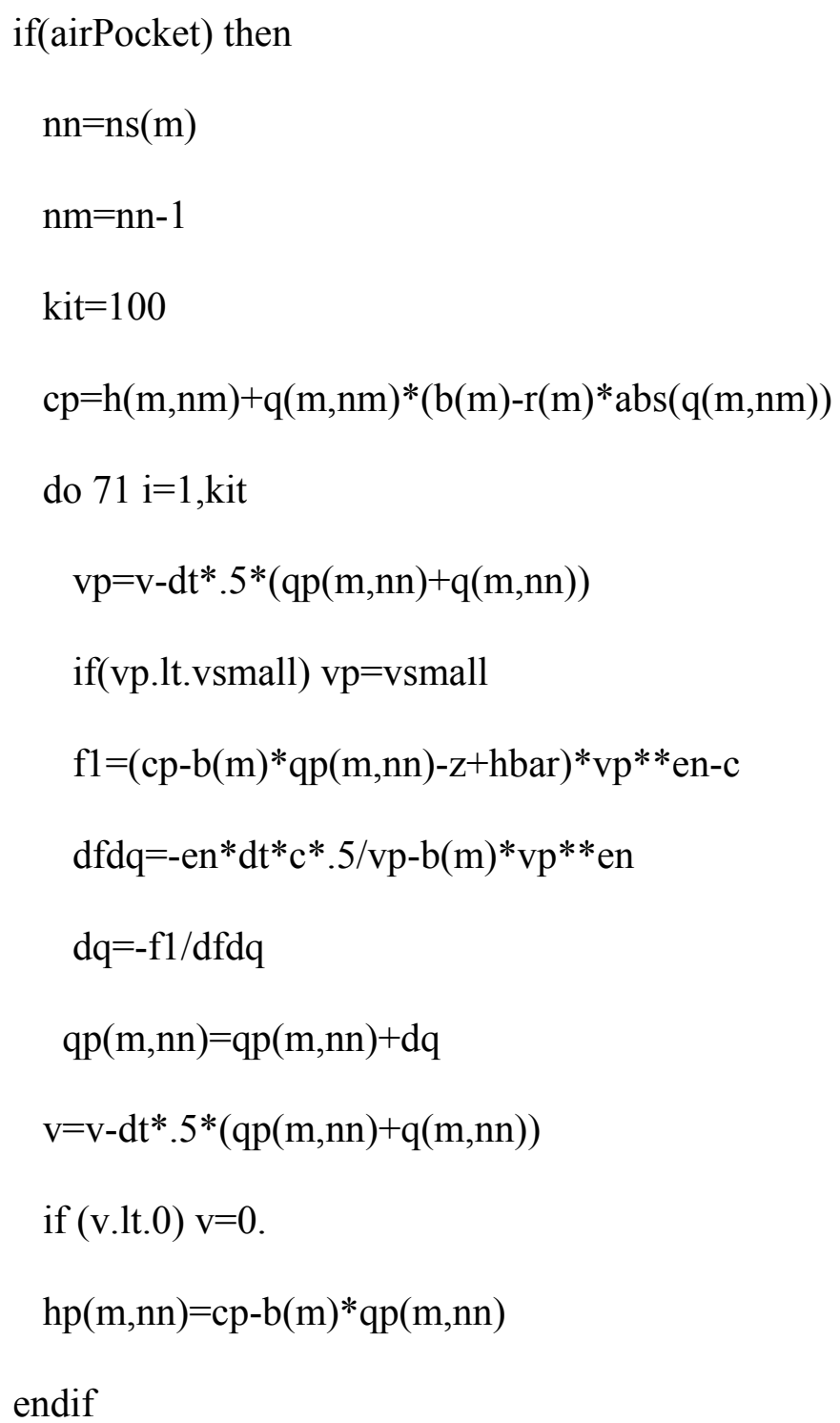

